\title{
Robust LIDAR-Based Localization in Underground Mines
}

\section{Kristin Nielsen}





\section{Robust LIDAR-Based}

Localization in Underground Mines

\section{Kristin Nielsen}


This is a Swedish Licentiate's Thesis.

Swedish postgraduate education leads to a Doctor's degree and/or a Licentiate's degree.

A Doctor's Degree comprises 240 ECTS credits (4 years of full-time studies).

A Licentiate's degree comprises 120 ECTS credits,

of which at least 60 ECTS credits constitute a Licentiate's thesis.

\begin{tabular}{|l|l}
\hline$(\mathrm{cc})$ EY-NC \\
\hline
\end{tabular} This work is licensed under a Creative Commons AttributionNonCommercial 4.0 International License.

https://creativecommons.org/licenses/by-nc/4.0/

Linköping studies in science and technology. Licentiate Thesis

No. 1906

Robust LIDAR-Based Localization in Underground Mines

Kristin Nielsen

\author{
kristin.nielseneliu.se \\ www. control.isy.liu.se \\ Department of Electrical Engineering \\ Linköping University \\ SE-581 83 Linköping \\ Sweden \\ ISBN 978-91-7929-642-1ＩSSN 0280-7971 \\ Copyright (C) 2021 Kristin Nielsen \\ Printed by LiU-Tryck, Linköping, Sweden 2021
}


To family and friends, 



\section{Abstract}

The mining industry is currently facing a transition from manual operation to remote or semi-automated control. The vision is fully autonomous vehicles being part of a larger fleet, with humans only setting high-level goals for the autonomous fleet to execute in an optimal way. An enabler for this vision is the presence of robust, reliable and highly accurate localization. This is a requirement for having areas in a mine with mixed autonomous vehicles, manually operated vehicles, and unprotected personnel. The robustness of the system is important from a safety as well as a productivity perspective. When every vehicle in the fleet is connected, an uncertain position of one vehicle can result in the whole fleet begin halted for safety reasons.

Providing reliable positions is not trivial in underground mine environments, where there are no access to global satellite based navigation systems. Due to the harsh and dynamically changing environment, onboard positioning solutions are preferred over systems utilizing external infrastructure. The focus of this thesis is localization systems relying only on sensors mounted on the vehicle, e.g., odometers, inertial measurement units, and 2D LIDAR sensors. The localization methods are based on the Bayesian filtering framework and estimate the distribution of the position in the reference frame of a predefined map covering the operation area. This thesis presents research where the properties of 2D LIDAR data, and specifically characteristics when obtained in an underground mine, are considered to produce position estimates that are robust, reliable, and accurate.

First, guidelines are provided for how to tune the design parameters associated with the unscented Kalman filter (UKF). The UKF is an algorithm designed for nonlinear dynamical systems, applicable to this particular positioning problem. There exists no general guidelines for how to choose the parameter values, and using the standard values suggested in the literature result in unreliable estimates in the considered application. Results show that a proper parameter setup substantially improves the performance of this algorithm.

Next, strategies are developed to use only a subset of available measurements without losing quality in the position estimates. LIDAR sensors typically produce large amounts of data, and demanding real-time positioning information limits how much data the system can process. By analyzing the information contribution from each individual laser ray in a complete LIDAR scan, a subset is selected by maximizing the information content. It is shown how $80 \%$ of available LIDAR measurements can be dropped without significant loss of accuracy.

Last, the problem of robustness in non-static environments is addressed. By extracting features from the LIDAR data, a computationally tractable localization method, resilient to errors in the map, is obtained. Moving objects, and tunnels being extended or closed, result in a map not corresponding to the LIDAR observations. State-of-the-art feature extraction methods for 2D LIDAR data are identified, and a localization algorithm is defined where features found in LIDAR data are matched to features extracted from the map. Experiments show that regions of the map containing errors are automatically ignored since no matching features are found in the LIDAR data, resulting in more robust position estimates. 



\section{Populärvetenskaplig sammanfattning}

Gruvindustrin befinner sig just nu i ett paradigmskifte. I en industri där mycket av arbetsuppgifterna traditionellt utförts manuellt ser man nu att människor steg för steg ersätts av automatiseringar. Istället för att ha en dedikerad operatör för varje enskild maskin, fordon, eller borrigg, finns nu en vision om en helt autonom flotta av maskiner där människor bara interagerar genom att sätta mål på betydligt högre nivå. En nödvändighet för att garantera både säkerhet och produktivitet $i$ en sådan vision är ett robust, pålitligt, och noggrant lokaliseringssystem. När alla enheter i flottan är sammankopplade kan en positionsosäkerhet på ett enda fordon få konsekvensen att hela flottan stannas för att garantera säkerheten. Det leder till stora produktivitetsförluster och det är därför av största vikt att lokaliseringssystemet är pålitligt och levererar positionsskattningar i realtid, samtidigt som det måste vara robust mot de förändringar som kontinuerligt sker i en gruvmiljö.

Att tillhandahålla ett sådant system är inte trivialt i en underjordsgruva där globala satellitnavigeringssystem inte är tillgängliga. Miljön kan vara fuktig, dammig, smutsig, extremt varm, eller extremt kall, och dessutom förändras den ständigt. Därför är ett lokaliseringssystem där maskinen själv skattar sin position att föredra framför ett system beroende av extern infrastruktur. Den här avhandlingen fokuserar på lösningar som endast använder odometri och hastighets-, accelerations-, och 2D laser-sensorer (LIDAR), monterade på fordonet. All sensordata innehåller någon form av brus och informationen från de olika sensorerna viktas samman till en enda positionsskattning med hänsyn till detta. Fordonets position skattas kontinuerligt, när ny sensordata är tillgänglig, genom att först prediktera en positon givet föregående positionsskattning och nuvarande hastighets- och accelerationsmätningar. Lasersensorerna mäter avståndet från sensorn till närliggande väggar och objekt i olika riktningar. Dessa mätningar används därefter för att justera den predikterade positionen. Genom att jämföra förväntade lasermätningar i den givna positionen givet en karta över området, med faktiska observationer från LIDAR-sensorn beräknas en skattning av fordonets position. I den här avhandlingen presenteras forskning där egenskaper i 2D LIDAR-data, och specifikt karaktärsdrag i datat som gruvmiljön ger upphov till, tas i beaktning för att leverera en robust och precis positionsskattning.

En algoritm som kan appliceras på det här problemet är ett unscented Kalman filter. Kopplat till algoritmen finns designparametrar, men bra riktlinjer för hur värden på dessa parametrar ska väljas saknas. Första delen av avhandlingen presenterar direktiv för hur lämpliga värden kan erhållas, och hur dessa parametervärden är helt avgörande för att få en rimlig positionsskattning i en gruvapplikation.

Vidare tar avhandlingen upp problemet att trots begränsad beräkningskapacitet, leverera skattningar i realtid. LIDAR-sensorer producerar mycket data och för att hinna processa datat utvecklas strategier för att välja ut en delmängd av tillgängliga mätningar utan att kvalitén på positionsskattningen påverkas.

Slutligen behandlas problemet med robusthet i en föränderlig miljö. Objekt i miljön som rör sig, eller nya gruvgångar, resulterar i en karta som inte stäm- 
mer överens med verkligheten. Genom att identifiera punkter i kartan och LIDARdatat där kurvaturen är stor, och sedan matcha dessa punkter mot varandra, fås en positionsskattning som automatiskt är robust mot fel i kartan. Resultat från simuleringar visar att med detta angreppssätt får man inga matchande punkter, och därmed inga mätningar, i regioner där kartan är felaktig. Till skillnad från när varje laserstråle behandlas för sig, matas då ingen falsk information vidare till lokaliseringsalgoritmen, och positionsskattningen blir mer robust. 


\section{Acknowledgments}

Many people have been involved in this PhD journey so far, and with these words I would like to recognize the most important ones. First of all, I want to thank my supervisor Gustaf Hendeby for guiding me in the academic jungle, always ready with useful advises, ideas, and invaluable feedback. Thank you for all the support and for always being available for discussions. Thanks also to my cosupervisor Fredrik Gustafsson for providing suggestions of research directions to take.

I also want to thank my industrial supervisor Robert Lundh for helping me with the Epiroc-perspective and for always believing in me. Without your and Lars Eriksson's positive attitude and support regarding this project, I would never have started this journey.

I would like to thank all my colleagues in both academia and industry for providing an inspiring work environment, and special thanks are in place to all that have proofread, all or parts, of this thesis. Anton Kullberg, Magnus Malmström, Per Boström, Robin Forsling, Jonas Nordlöf, Erik Jakobsson, Max Åstrand, and John Svensson, your comments and suggestions have been much appreciated.

This work was partially supported by the Wallenberg AI, Autonomous Systems and Software Program (WASP) funded by the Knut and Alice Wallenberg Foundation. Their funding is gratefully acknowledged. WASP also introduced me to a large number of inspiring people, where Caroline Svahn and Hector Rodriguez Deniz have become my extended academic family. Thank you, also to the mining cluster, Erik Jakobsson, Max Åstrand, and John Svensson, for so friendly letting me be a part of your already established community.

The remaining part of the funding of this work comes from Epiroc Rock Drills $A B$, for which I am grateful, and especially so to Mats Källman for removing the burden of financial details.

Finally, I would like to express my deepest gratitude to my family for supporting me in this project. I am grateful for my kids, Agnes, Ebba, and Elvira, without you this thesis would probably have taken a year less to complete. Thank you also to my dad and mum, Leif and Elisabet, for your ground service during my many trips to Linköping. Last, but definitely not the least, I want to send a tremendous thank you to my husband Tommy. Without you I would not have reached this far.

Karlskoga, May 2021

Kristin Nielsen 



\section{Contents}

1 Introduction 1

1.1 Background and motivation $\ldots \ldots \ldots \ldots \ldots \ldots$

1.2 Research questions . . . . . . . . . . . . . 3

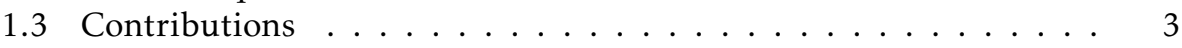

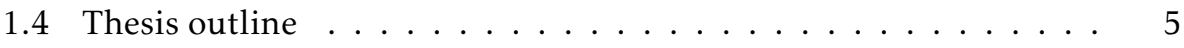

2 Background 7

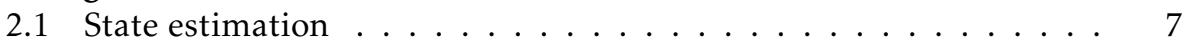

2.1.1 State-space models . . . . . . . . . . . . . . 7

2.1 .2 Filtering theory $\ldots \ldots \ldots \ldots \ldots$

2.1 .3 Extended Kalman filter . . . . . . . . . . . . . . . . . . . 11

2.1.4 Unscented Kalman filter . . . . . . . . . . . . . . . . . 11

2.1.5 Monte Carlo filter . . . . . . . . . . . . . . . . . . 13

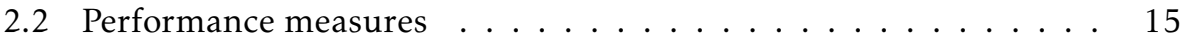

2.2.1 Cramér-Rao lower bound . . . . . . . . . . . . . . . . 15

2.2.2 Information measures . . . . . . . . . . . . . 16

2.2 .3 Log-likelihood . . . . . . . . . . . . . . . . . . 17

2.32 D LIDAR sensors . . . . . . . . . . . . . . . . . . . . . 17

2.3.1 Ray measurement model . . . . . . . . . . . . . 18

2.4 Scan registration . . . . . . . . . . . . . . . . . . 21

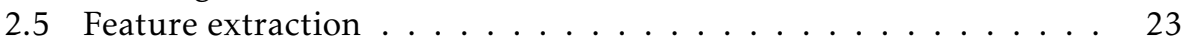

2.5 .1 Sensor data . . . . . . . . . . . . . . . . . 24

2.5 .2 Detectors . . . . . . . . . . . . . . . 24

2.5 .3 Descriptors . . . . . . . . . . . . . . . . . . . . . . . . . 29

2.5.4 Data association . . . . . . . . . . . . . 31

3 Design parameters in the unscented Kalman filter 33

3.1 Unscented transformation . . . . . . . . . . . . . . 34

3.1 .1 Sigma point set $\ldots \ldots \ldots \ldots \ldots \ldots \ldots \ldots$

3.1 .2 UT parameters . . . . . . . . . . . . . . . 35

3.2 Design parameter guidelines . . . . . . . . . . . . . 37

3.2.1 Measurement function example . . . . . . . . . . . . 37

3.2 .2 Guidelines . . . . . . . . . . . . . . . . 38 


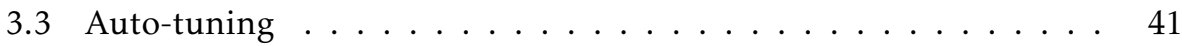

3.4 Simulations . . . . . . . . . . . . . . . . . . . 41

3.4 .1 Simplified example . . . . . . . . . . . . . . 42

3.4 .2 Real data . . . . . . . . . . . . . . . . . 45

3.5 Summary . . . . . . . . . . . . . . . . . . . 49

4 Sensor selection in 2D LIDAR data 51

4.1 Map representation . . . . . . . . . . . . . . . 51

4.1.1 LIDAR measurement model . . . . . . . . . . . . 52

4.1.2 Imperfect map representation . . . . . . . . . . . . 53

4.2 Information in LIDAR measurements . . . . . . . . . . . . . 53

4.2 .1 Information from individual rays . . . . . . . . . . 54

4.2 .2 Imperfect map . . . . . . . . . . . . . . . . . 54

4.2 .3 Map corner points . . . . . . . . . . . . . . . . 55

4.3 Sensor selection methods . . . . . . . . . . . . . . 56

4.3.1 Sensor selection by optimization $\ldots \ldots \ldots \ldots$

4.3.2 Uniformly distributed (UNI) . . . . . . . . . . . . . 57

4.3.3 Greedy with adjacent rays (GAR) . . . . . . . . . . 57

4.3.4 Local difference in measurement data (LDM) . . . . . . 58

4.4 Experiments . . . . . . . . . . . . . . . . . 60

4.4 .1 Setup . . . . . . . . . . . . . . 60

4.4 .2 Simplified map . . . . . . . . . . . . . . 61

4.4 .3 Realistic map . . . . . . . . . . . . . . . . . . 61

4.4 .4 Map with errors . . . . . . . . . . . . . . . . . 63

4.5 Summary . . . . . . . . . . . . . . . . . . 64

5 Feature extraction in 2D LIDAR Data $\quad 67$

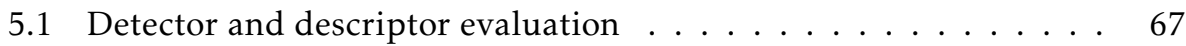

5.1 .1 Evaluation data . . . . . . . . . . . . . 68

5.1 .2 Adaptations to map positioning . . . . . . . . . 68

5.1 .3 Detector quality measures . . . . . . . . . . . . . . 69

5.1 .4 Descriptor quality measures . . . . . . . . . . . 72

5.2 Underground positioning application . . . . . . . . 72

5.2.1 Pose estimation from feature points . . . . . . . . . 73

5.2.2 Direct scan matching . . . . . . . . . . . . . 75

5.3 Positioning experiments $\ldots \ldots \ldots \ldots \ldots \ldots$

5.3.1 Measurement update . . . . . . . . . . . . 76

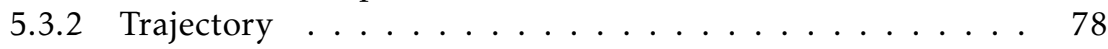

5.4 Summary . . . . . . . . . . . . . . . 82

6 Concluding remarks 83

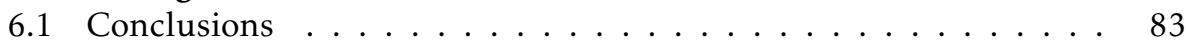

6.2 Future work $\ldots \ldots \ldots \ldots \ldots \ldots \ldots \ldots$

$\begin{array}{ll}\text { Bibliography } & 85\end{array}$ 


\section{1}

Introduction

The mining industry is facing a paradigm shift, where much of the operations traditionally performed manually by humans are now step by step being automated. A robust and highly accurate positioning system is in many cases a requirement for this transition to happen. Positioning in the harsh, unstructured and global navigation satellite system (GNSS) denied environment of an underground mine is the topic of this thesis, and focus is on onboard systems utilizing 2D LIDAR sensors, without requirements of external infrastructure. This introductory chapter gives an overview of the underground localization problem, lists the contributions, and outlines the content of this thesis.

\subsection{Background and motivation}

As late as 2002, it was stated in [90] that "Where are we? In a tunnel!" was enough positioning information for mining, as one would anyhow follow the tunnel until the next intersection, and then make decisions and take action. However, in the last decade, as autonomously operated vehicles have become a real option to improve safety and increase productivity in mines, a need for more accurate positioning is arising. Current modern mining localization practice mainly focuses on; personal safety and emergency rescue, where knowledge of who is in the mine and at what level is enough [105], and, knowledge of in which area machines are located to identify ventilation needs. However, a real-time, robust, and highly accurate localization system would have the potential to significantly improve safety for present human workers, contribute to the advancement of automated vehicle systems, and enable the deployment of productivity improving fleet management systems for underground operations [3].

Underground mining is an industrial area where automation technologies are less developed than for similar industries above ground [104]. An underground 


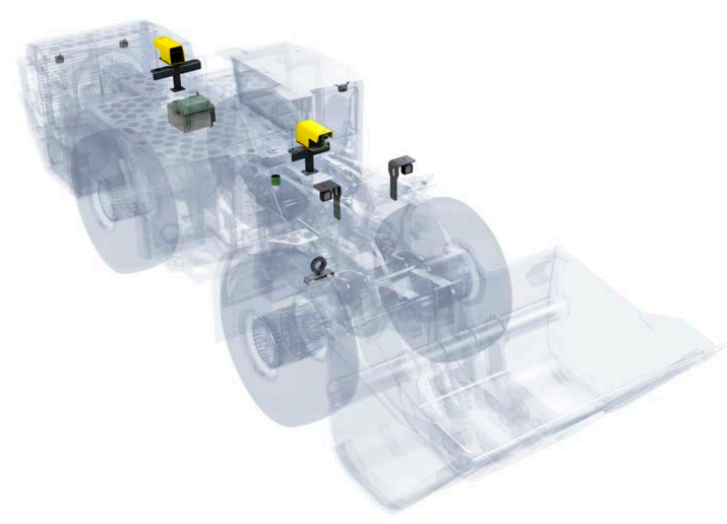

Figure 1.1: Epiroc underground loader showing where LIDAR sensors are mounted. Asset: Epiroc

mine presents unique challenges, as GNSS measurements are not available and the operation area is not the open space of a typical outdoor scenario. Neither can it be represented by the classical clean indoor environment with smooth walls and structured surroundings [69] [23]. The environment in an underground mine also changes dynamically as the result of tunnels being extended or closed, corners being worn, or wall reinforcements. Accurate map creation is considered in [3]. In this thesis the map is assumed known beforehand, but the effects of map inaccuracies are considered.

The tunnel network in underground mines is typically very large compared to the number of vehicles operating in it, and maintenance of an external infrastructure dedicated for positioning would be expensive. The harsh, unstructured, and highly dynamic environment also favors as little infrastructure as possible and an onboard localization system is therefore preferred [70]. Today, autonomous operation in underground mines is possible for specific tasks and preconditions, e.g., loaders navigating along prerecorded paths are commercially available from Sandvik, Caterpillar, and Epiroc [81]. These systems do not require global positioning due the recordings of the environment, which often is a time consuming and cumbersome task to perform. However, they have local positioning systems to navigate along the path, that rely on LIDAR sensors, odometry, and data from inertial measurement units (IMU). See Figure 1.1 for sensor placements on an autonomous loader from Epiroc. Camera and radar sensors commonly used by similar systems for autonomous cars, are not suitable for underground applications; cameras since it is dark and dirty in an underground mine, limiting the visibility, and radar does not give enough precision for a large vehicle to navigate in a narrow tunnel, not much wider that the vehicle itself.

Dead reckoning based localization, using IMU and odometry, is a favorable solution to the positioning problem as it requires no external infrastructure and is robust to the dirty mine environment. However, dead reckoning inherently suffers from drift [57] and cannot be used alone for extended periods of time, but 
is an important component when fused with other information sources [78, 105].

WiFi network [39], radio frequency identification (RFID) $[64,71]$, and ultra wideband (UWB) $[1,18,105]$ based methods are often put forward for indoor positioning. WiFi and RFID have been reported to provide meter accuracy in office environments, but attempts with WiFi-based positioning in underground environments reports lower accuracy $[23,85]$. UWB is a promising technology due to its high precision (15 cm reported indoors [118]). However, metallic objects can cause disturbances [64] and sensitivity to optimal placement of the UWB anchors [4] limits the applicability in mines.

Another possible method to obtain global position estimates is to compare LIDAR measurements to a predefined map [22] utilizing sensor fusion and filtering theory. This has been used with good results indoors, and uses the same sensors as existing commercial autonomous mine solutions have. Hence, this is the solution considered in this thesis. Filtering theory provides a framework to recursively update an estimate of the position as new noisy measurements become available. Both an estimate of the state of the system, in this case comprising the position and orientation, as well as an estimate of the uncertainty of the state is provided. The well-known Kalman filter (KF) is the optimal state estimator if the problem is linear with Gaussian noise, while the extended Kalman filter (EKF) or the unscented Kalman filter (UKF) are two commonly used state estimators for the nonlinear case.

\subsection{Research questions}

The ultimate goal of this work is to provide reliable and highly accurate positioning in underground mines. The solution needs to operate in real time, be insensitive to changes in the environment, and should preferably make use of sensor hardware currently available on semi-autonomous mining vehicles, such as 2D LIDAR. More specifically, this thesis considers the following:

- What can be done to reduce the computational complexity of standard positioning algorithms? In particular, can something be done about the high dimensionality of the LIDAR measurements?

- How can the robustness of the localization system be improved? Robustness against errors in the map or unknown changes in the environment, but also robustness in the positioning algorithms to handle the demanding underground mine environment.

\subsection{Contributions}

The main contributions of this thesis are presented in Section 2.5, and Chapter 3, 4 and 5, respectively, and are the following:

- UKF parameter tuning, considered in Chapter 3. Despite the knowledge that the UKF can have very poor performance when the parameters are im- 
properly chosen, there exist no rule-of-thumbs for how to tune them. By analyzing the effect of each of the parameters, guidelines are provided for how the parameters should be tuned. The guidelines are verified both in simulations and on real data collected in an underground mine. The experiments also show that with properly chosen parameter values the performance of the UKF can be substantially improved.

This work started of as a joint project between Epiroc and several institutions at Linköping university under the umbrella of the WASP project course. The material has after the end of the course been extended with a literature survey of the problem, highlighting examples, and a more in-depth analysis of the significance of the parameters. The author of this thesis has been the main driving force in this extension. A manuscript, primarily written by the author of this thesis, has been submitted to Automatica, and is currently under review.

- Sensor Selection in 2D LIDAR data, studied in Chapter 4. Methods for selecting subsets of available measurements in a LIDAR scan are developed by analyzing the information contribution from each individual laser ray. The analysis shows what situations and aspects that are important for LIDAR measurements to be informative. The methods limit the computations needed while maintaining the quality of the position estimates.

This work was presented at 2020 International Conference on Information Fusion.

K. Nielsen and G. Hendeby. Sensor management in 2D lidarbased underground positioning. In IEEE $23^{\text {th }}$ Proceedings of the International Conference on Information Fusion, pages 1-6, Virtual conference, July 2020. C2020 IEEE.

The author of this thesis has written the manuscript, performed the analysis, and conducted the experiments, while the underlying ideas are a joint effort by the author and co-author.

- Feature Extraction in 2D LIDAR data. A survey on feature extraction methods specialized on 2D LIDAR data is presented in Section 2.5. In Chapter 5 adaptations are made to state-of-the-art 2D LIDAR feature extraction methods, to fit the problem of positioning in a predefined map. The methods are evaluated through state estimation experiments in a simulated mine environment, with results indicating that the feature extraction approach makes the state estimate much more robust to changes and errors in the map.

A manuscript comprising this work has been submitted to IEEE Transactions on Automation Science and Engineering, and is currently under review. The author of this thesis has been the main driving force in the literature study, evaluation and writing of the manuscript. The co-author has supported through discussions, thoughts, and ideas. 


\subsection{Thesis outline}

This thesis is organized as follows:

- Chapter 1 (this chapter) introduces the research problem and highlights the contributions of this thesis.

- Chapter 2 gives an overview of the theoretical frameworks used in this thesis. It introduces state estimation by filtering theory and performance measures for how to evaluate results. Properties of 2D LIDAR data are presented as well as preprocessing strategies of LIDAR data to improve the state estimate. A survey on feature extraction methods specialized on $2 \mathrm{D}$ LIDAR data is also presented.

- Chapter 3 is based on the manuscript regarding UKF parameter tuning. Guidelines and an optimization technique for how to choose design parameters in a UKF to obtain good state estimates is developed. Results are presented from a simulated example as well as from real data recorded in an underground mine environment.

- Chapter 4 is based on the publication [77] and considers the problem of selecting a subset of available measurements from a 2D LIDAR scan. To decrease processing time, without losing performance of the resulting state estimate, the information contribution from each individual laser ray is analyzed.

- Chapter 5 is based on the manuscript about feature extraction methods. State-of-the-are feature extraction methods for 2D LIDAR data are in this chapter incorporated in the state estimation procedure. Experiments from the underground mine scenario show that this preprocessing of the LIDAR data makes the state estimate more robust to errors in the map.

- Chapter 6 concludes the thesis and gives suggestions for future work. 



\section{2}

\section{Background}

This chapter contains the background theory for this thesis. Section 2.1 gives an overview of statistical models for state estimation in dynamical systems, while Section 2.2 briefly introduces performance measures for state estimation. Properties of a 2D LIDAR sensor is presented in Section 2.3, and Section 2.5 presents a survey on feature extraction methods specifically designed for 2D LIDAR data.

\subsection{State estimation}

This section provides an introduction to Bayesian filtering and how it is used to estimate the state vector in a dynamic system with stochastic properties.

\subsubsection{State-space models}

A discrete state-space model is a mathematical representation of a system describing the relationship between the input, noise, and output signals, written as a discrete difference model originating from a system of first-order differential equations $[32,65]$. This system representation is a natural choice for state estimation purposes since the state vector is explicitly modeled. In a probabilistic framework a general state-space model formulation in discrete time is given by [97],

$$
\begin{aligned}
& \mathbf{x}_{0} \sim p\left(\mathbf{x}_{0}\right), \\
& \mathbf{x}_{k} \sim p\left(\mathbf{x}_{k} \mid \mathbf{x}_{k-1}, \mathbf{u}_{k-1}\right), \\
& \mathbf{y}_{k} \sim p\left(\mathbf{y}_{k} \mid \mathbf{x}_{k}, \mathbf{u}_{k}\right),
\end{aligned}
$$$$
\text { for } k=1,2, \ldots
$$

where $\mathbf{x}_{k} \in \mathbb{R}^{n}$ is the state of the system, $\mathbf{y}_{k} \in \mathbb{R}^{m}$ are the observations and $\mathbf{u}_{k}$ are known exogenous inputs to the system, all at time step $k$. The distribution 
$p\left(\mathbf{x}_{k} \mid \mathbf{x}_{k-1}, \mathbf{u}_{k-1}\right)$ is the state transition model of the system, describing the stochastic dynamics of the system by a conditional probability function, and $p\left(\mathbf{y}_{k} \mid \mathbf{x}_{k}, \mathbf{u}_{k}\right)$ is the measurement model, which models the likelihood of an observation given the state.

A state-space model is assumed to be Markovian which means that,

- $\mathbf{x}_{k}$ given $\mathbf{x}_{k-1}$ is independent of anything that happened before time step $k-1$,

- the observation $\mathbf{y}_{k}$ only depends on the current state $\mathbf{x}_{k}$ (and possibly also the input $\mathbf{u}_{k}$ ).

Often the observations are also independent of the input, further simplifying (2.1c) to $p\left(\mathbf{y}_{k} \mid \mathbf{x}_{k}\right)$.

As an alternative to the probabilistic formulation of the state-space model in (2.1) a slightly more restrictive functional representation is commonly used

$$
\begin{aligned}
& \mathbf{x}_{k}=\mathbf{f}\left(\mathbf{x}_{k-1}, \mathbf{u}_{k-1}, \mathbf{v}_{k}\right), \\
& \mathbf{y}_{k}=\mathbf{h}\left(\mathbf{x}_{k}, \mathbf{u}_{k}, \mathbf{e}_{k}\right),
\end{aligned}
$$

where the state transition function $\mathbf{f}$, and the measurement function $\mathbf{h}$ are arbitrary nonlinear functions. The process noise and measurement noise denoted by, $\mathbf{v}_{k}$ and $\mathbf{e}_{k}$, respectively, are assumed mutually independent. An important special case of (2.2) occurs when the noise enters the model additively, yielding the additive state-space model,

$$
\begin{aligned}
& \mathbf{x}_{k}=\mathbf{f}\left(\mathbf{x}_{k-1}, \mathbf{u}_{k-1}\right)+\mathbf{v}_{k}, \\
& \mathbf{y}_{k}=\mathbf{h}\left(\mathbf{x}_{k}, \mathbf{u}_{k}\right)+\mathbf{e}_{k} .
\end{aligned}
$$

In the probabilistic formulation of the state-space model this is equivalent to,

$$
\begin{aligned}
p\left(\mathbf{x}_{k} \mid \mathbf{x}_{k-1}, \mathbf{u}_{k-1}\right) & =p_{\mathbf{v}}\left(\mathbf{x}_{k}-\mathbf{f}\left(\mathbf{x}_{k-1}, \mathbf{u}_{k-1}\right)\right), \\
p\left(\mathbf{y}_{k} \mid \mathbf{x}_{k}, \mathbf{u}_{k}\right) & =p_{\mathbf{e}}\left(\mathbf{y}_{k}-\mathbf{h}\left(\mathbf{x}_{k}, \mathbf{u}_{k}\right)\right),
\end{aligned}
$$

where $p_{\mathbf{v}}(\mathbf{x}), p_{\mathbf{e}}(\mathbf{x})$ are the probability density functions of the process noise and measurement noise, respectively.

A commonly used assumption is that the noise distributions are white Gaussian,

$$
\begin{aligned}
& \mathbf{v}_{k} \sim \mathcal{N}\left(\mathbf{0}, \mathbf{Q}_{k}\right), \\
& \mathbf{e}_{k} \sim \mathcal{N}\left(\mathbf{0}, \mathbf{R}_{k}\right),
\end{aligned}
$$

and that the initial state has a Gaussian distribution

$$
\mathbf{x}_{0} \sim \mathcal{N}\left(\overline{\mathbf{x}}_{0}, \mathbf{P}_{0}\right)
$$

with $\mathbf{Q}_{k}, \mathbf{R}_{k}$ and $\mathbf{P}_{0}$ being positive semi-definite covariance matrices. 


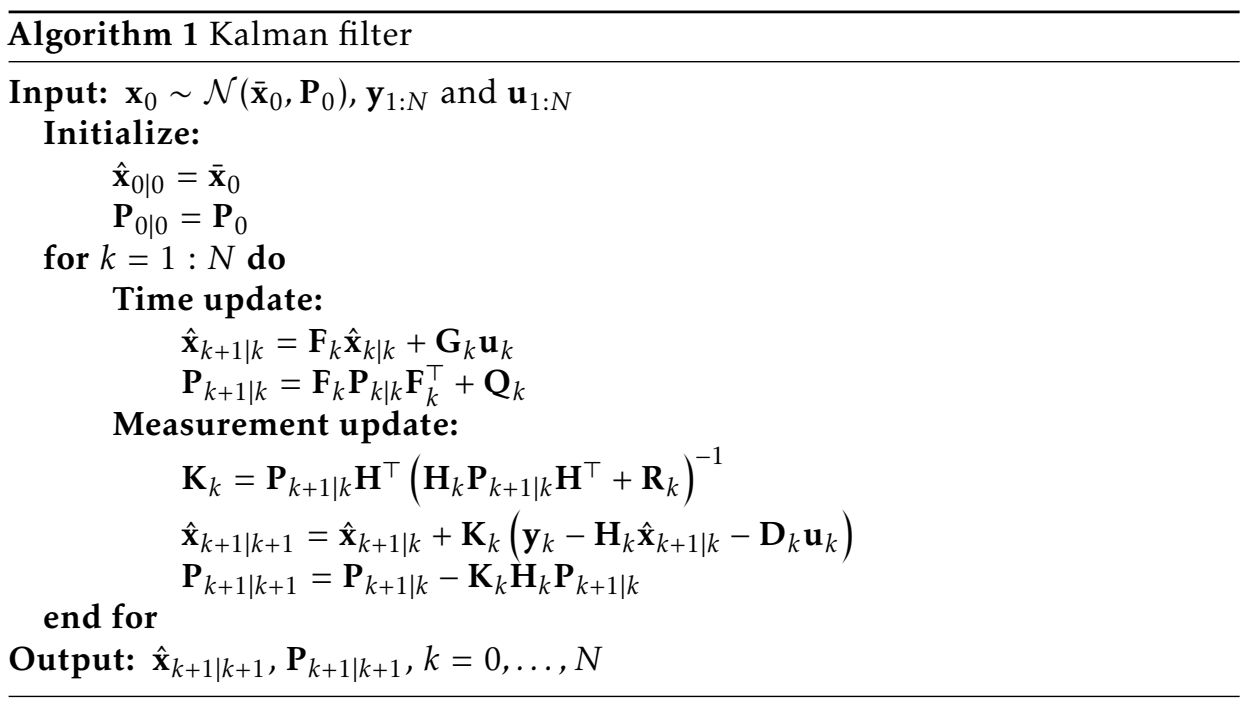

\subsubsection{Filtering theory}

Filtering theory addresses the problem of estimating the state of a system from noisy observations $[97,104]$. With the probabilistic formulation of the statespace model in (2.1), the problem consists of estimating the marginal posterior distribution

$$
p\left(\mathbf{x}_{k} \mid \mathbf{y}_{1: k}\right) \text {. }
$$

That is, to compute the distribution of $\mathbf{x}_{k}$ at each time step $k$ given all observations up to this time step, denoted by $\mathbf{y}_{1: k}$. With the Markovian assumption this can be done recursively by iterating between a time update, also known as the prediction step, and a measurement update [104].

- Time update:

$$
p\left(\mathbf{x}_{k} \mid \mathbf{y}_{1: k-1}\right)=\int p\left(\mathbf{x}_{k} \mid \mathbf{x}_{k-1}\right) p\left(\mathbf{x}_{k-1} \mid \mathbf{y}_{1: k-1}\right) d \mathbf{x}_{k-1} .
$$

- Measurement update:

$$
p\left(\mathbf{x}_{k} \mid \mathbf{y}_{1: k}\right)=\frac{p\left(\mathbf{y}_{k} \mid \mathbf{x}_{k}\right) p\left(\mathbf{x}_{k} \mid \mathbf{y}_{1: k-1}\right)}{p\left(\mathbf{y}_{k} \mid \mathbf{y}_{1: k-1}\right)},
$$

with the normalization constant given by

$$
p\left(\mathbf{y}_{k} \mid \mathbf{y}_{1: k-1}\right)=\int p\left(\mathbf{y}_{k} \mid \mathbf{x}_{k}\right) p\left(\mathbf{x}_{k} \mid \mathbf{y}_{1: k-1}\right) d \mathbf{x}_{k}
$$

In general this Bayesian recursion cannot be computed in closed form and analytical solutions only exist for a few special cases. The well-known Kalman 


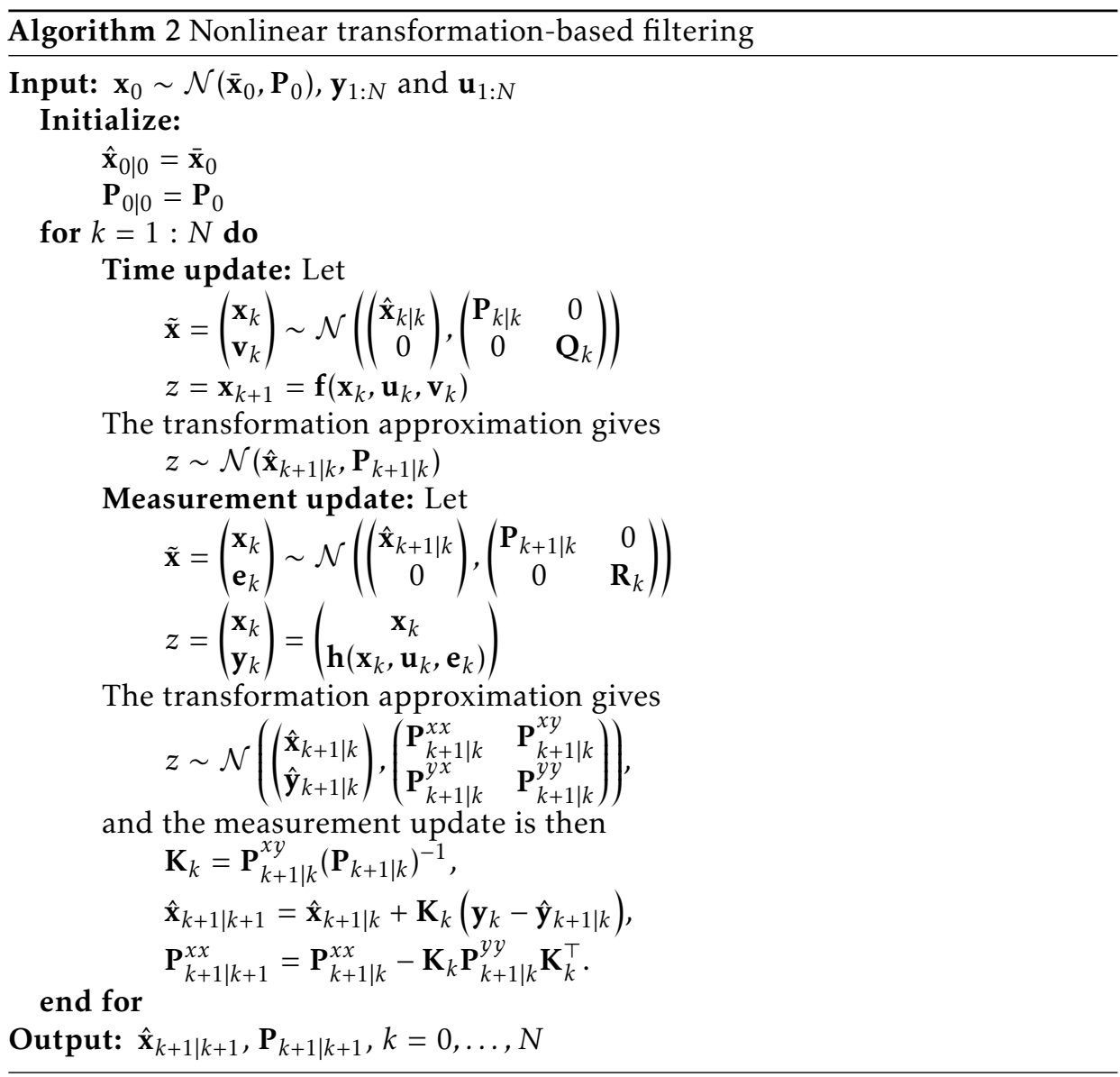

filter (KF), originally derived in [51], analytically solves one such case, that of a linear state-space model with additive Gaussian noise. This corresponds to the additive state-space model (2.3), with $\mathbf{f}\left(\mathbf{x}_{k-1}, \mathbf{u}_{k-1}\right)=\mathbf{F}_{k-1} \mathbf{x}_{k-1}+\mathbf{G}_{k-1} \mathbf{u}_{k-1}$ and $\mathbf{h}\left(\mathbf{x}_{k}, \mathbf{u}_{k}\right)=\mathbf{H}_{k} \mathbf{x}_{k}+\mathbf{D}_{k} \mathbf{u}_{k}$, where $\mathbf{F}_{k-1}, \mathbf{G}_{k-1}, \mathbf{H}_{k}$ and $\mathbf{D}_{k}$ are matrices. For a complete derivation of the KF see for example [97]. Algorithm 1 outlines the KF with the state estimate $\hat{\mathbf{x}}_{k_{1} \mid k_{2}}$, given by the Bayesian filter equations with

$$
p\left(\mathbf{x}_{k_{1}} \mid \mathbf{y}_{1: k_{2}}\right)=\mathcal{N}\left(\mathbf{x}_{k_{1}} \mid \hat{\mathbf{x}}_{k_{1} \mid k_{2}}, \mathbf{P}_{k_{1} \mid k_{2}}\right),
$$

where the notation $\hat{\mathbf{x}}_{k_{1} \mid k_{2}}$, indicates the state estimate at time step $k_{1}$ given measurements up to time step $k_{2}$.

In the case of a nonlinear state-space model, a closed form solution generally does not exist. However, by constructing nonlinear transformations that can be numerically approximated, the framework of the Kalman filter can be applied to recursively estimate the state also for nonlinear state-space models. This is formalized in the nonlinear transformation based filtering method outlined in Algorithm 2 [33]. This is a neat formulation of the filtering problem since the 
method of transformation approximation is interchangeable. There is a possibility to mix approximations in the time and measurement update even though most often the same approximation is used in both updates. The following sections describe three possible choices of transformation approximation methods. Note that all three methods are also able to approximate distributions that are non-Gaussian. It is therefore possible to apply the filter resulting from each of the approximation methods also to non-Gaussian distributions.

\subsubsection{Extended Kalman filter}

The extended Kalman filter (EKF) implements the most straightforward choice of transformation approximation, which is a first order Taylor expansion. Consider the nonlinear mapping of a multivariate variable $\mathbf{x}$ with mean $\mu_{x}$ and covariance $\mathbf{P}_{x}$

$$
\mathbf{z}=\mathbf{h}(\mathbf{x})
$$

with a first order Taylor expansion around $\mu_{x}$

$$
\mathbf{z}=\mathbf{h}(\mathbf{x}) \approx \mathbf{h}\left(\mu_{x}\right)+\nabla_{\mathbf{x}} \mathbf{h}\left(\mu_{x}\right)\left(\mathbf{x}-\mu_{x}\right)
$$

where $\nabla_{\mathbf{x}} \mathbf{h}\left(\mu_{x}\right)$ denotes the Jacobian evaluated in $\mathbf{x}=\mu_{x}$. The mean $\mu_{z}$ and covariance $\mathbf{P}_{z}$ of $\mathbf{z}$ are then approximated by

$$
\begin{aligned}
& \mu_{z} \approx \mathbf{h}\left(\mu_{x}\right), \\
& \mathbf{P}_{z} \approx\left(\nabla_{\mathbf{x}} \mathbf{h}\left(\mu_{x}\right)\right) \mathbf{P}_{x}\left(\nabla_{\mathbf{x}} \mathbf{h}\left(\mu_{x}\right)\right)^{\top} .
\end{aligned}
$$

Figure 2.1(a) and (b), illustrates this approximation for a 2-dimensional state vector, and by using this in Algorithm 2 the EKF is obtained. For a complete derivation of the EKF see [97] where also a version of the algorithm using a second order Taylor expansion approximation is presented.

\subsubsection{Unscented Kalman filter}

The unscented Kalman filter (UKF) is obtained by utilizing the unscented transform (UT) as transformation approximation in Algorithm 2 [47, 48]. The idea of the UT is to approximate the mean and covariance of the prior distribution by a set of so called sigma points (SP's). These SP's are then transformed without approximation and the results are weighted together to form the posterior distribution.

Let us again consider the nonlinear mapping in (2.12), and denote the set of $p$ SP's as

$$
\mathcal{X}^{(i)}, \quad i=0, \ldots, p-1,
$$

with associated weights

$$
\omega^{(i)}, \quad i=0, \ldots, p-1 .
$$




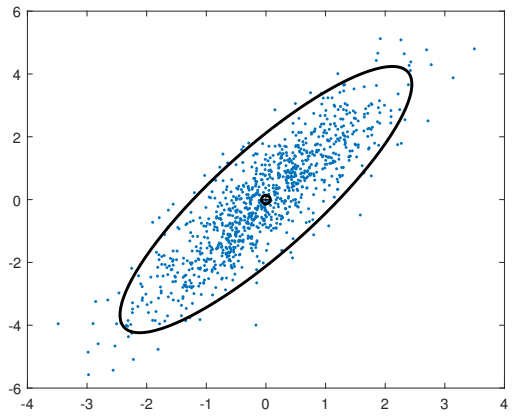

(a) Original distribution with mean and covariance.

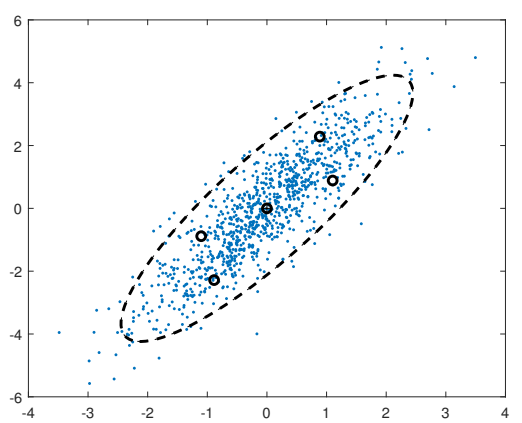

(c) Original distribution and sigma points according to the unscented transform marked as small circles. The covariance of the original distribution and the sigma points coincides and is represented by the dashed line.

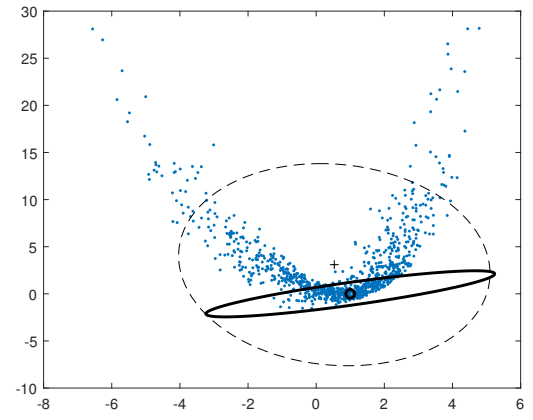

(b) Transformed distribution. The covariance of the true distribution is presented by the dashed line and the solid line is the approximation by first order Taylor expansion.

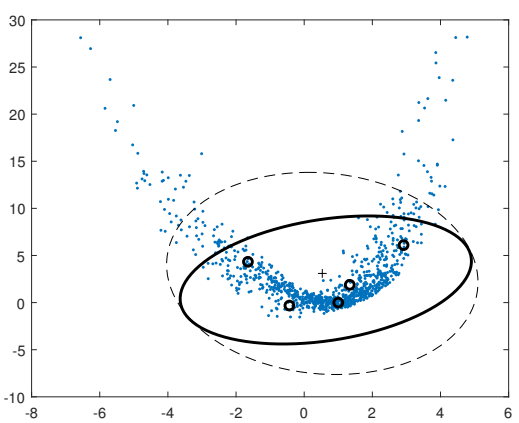

(d) Transformed distribution. The covariance of the true distribution is presented by the dashed line and the solid line is the approximation obtained by propagating the sigma points through the nonlinear function.

Figure 2.1: Illustration of the Taylor expansion approximation and the unscented transform for a nonlinear transformation. The state is given by $\mathbf{x}=\left[\begin{array}{l}x_{1} \\ x_{2}\end{array}\right]$, and the transformation by $\mathbf{h}(\mathbf{x})=\left[\begin{array}{c}\cos \left(x_{1}\right)+x_{2} \\ x_{2}^{2}+x_{1}\end{array}\right]$. The figures to the left show the original distribution before undergoing the transformation. To the right the distribution after the transformation is shown together with the first order Taylor approximation at the top and the UT approximation at the bottom. The true distributions are obtained by Monte Carlo sampling and transformation. 
The weights can be positive or negative, but to provide an unbiased estimate they must satisfy

$$
\sum_{i=0}^{p-1} \omega^{(i)}=1 .
$$

The posterior mean and covariance estimates are then given by

$$
\begin{aligned}
\mu_{z} & =\sum_{i=0}^{p-1} \omega_{m}^{(i)} \mathcal{Z}^{(i)}, \\
\mathbf{P}_{z} & =\sum_{i=0}^{p-1} \omega_{c}^{(i)}\left(\mathcal{Z}^{(i)}-\mu_{z}\right)\left(\mathcal{Z}^{(i)}-\mu_{z}\right)^{\top},
\end{aligned}
$$

with potentially different weights for the mean and covariance estimates and $\mathcal{Z}^{(i)}=\mathbf{h}\left(\mathcal{X}^{(i)}\right)$. Figure 2.1(c) and (d), illustrates the UT for a 2-dimensional state vector and the filter (UKF) obtained by using the UT as approximation method in Algorithm 2, is explicitly outlined in Algorithm 3 [115].

The standard strategy for selecting the SP's is to choose a set of $2 n_{x}+1$ points symmetric around the mean $\mu_{x}$, where $n_{x}=\operatorname{dim}(\mathbf{x})[47,115]$. The symmetric SP-set is obtained from

$$
\begin{array}{rlrl}
\mathcal{X}^{(0)} & =\mu_{x}, & \\
\mathcal{X}^{( \pm i)}=\mu_{x} \pm\left(\sqrt{\frac{n_{x}}{1-\omega^{(0)}} \mathbf{P}_{x}}\right)_{i}, & i=1, \ldots, n_{x}, \\
\omega^{( \pm i)}=\frac{1-\omega^{(0)}}{2 n_{x}}, & i=1, \ldots, n_{x},
\end{array}
$$

where $(\cdot)_{i}$ denotes the $i$ th column vector. The square root $\sqrt{\mathbf{P}_{x}}$ can be any matrix square root, but is usually implemented with the numerically efficient Cholesky factorization method [112]. The weight $\omega^{(0)}$ is associated with the mean sigma point $\mathcal{X}^{(0)}$ and is parameterized for tuning. Standard choices of parameterization and parameter values are suggested in early publications on UT/UKF $[42,44,45$, $47,48,115]$. There exists no general guidelines for how to choose the parameter values and in UKF implementations the values are often either left unchanged from the original suggestions or chosen ad hoc $[12,35,75,89,119,120]$. A more in-depth discussion on how to select SP's and how the design parameters influence the transformation approximation is provided in Chapter 3.

\subsubsection{Monte Carlo filter}

The Monte Carlo transformation (MCT) approximates the posterior statistics by sampling from the prior distribution and letting each sample pass through the nonlinear transformation [32]. The sample points $x^{(i)}$ are randomly selected, 


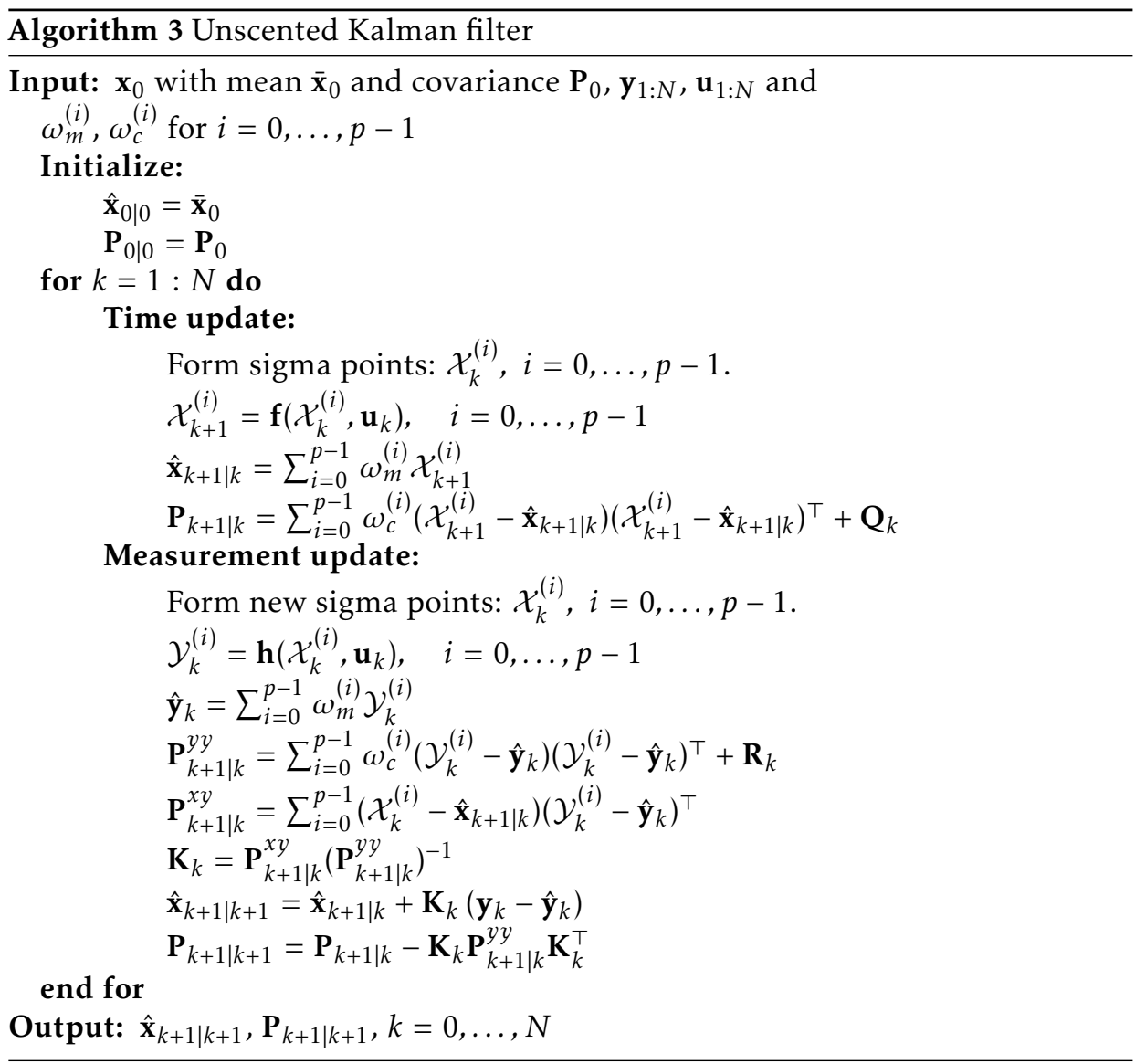

which differs from the UT method where the sigma point selection is deterministic. Considering the nonlinear mapping in (2.12), estimates of the posterior mean and covariance is then given by

$$
\begin{aligned}
\mathbf{z}^{(i)} & =\mathbf{h}\left(\mathbf{x}^{(i)}\right), \quad i=1, \ldots, N, \\
\mu_{z} & =\frac{1}{N} \sum_{i=1}^{N} \mathbf{z}^{(i)}, \\
\mathbf{P}_{z} & =\frac{1}{N-1} \sum_{i=1}^{N}\left(\mathbf{z}^{(i)}-\mu_{z}\right)\left(\mathbf{z}^{(i)}-\mu_{z}\right)^{T},
\end{aligned}
$$

where $N$ is the number of sampled points. This method does, under weak conditions, converge to the correct moments of the posterior distribution and asymptotically this should be the best possible approximation. The MCT is therefore used for validation in numerical comparisons in this thesis. See Figure 2.1 for an illustration of the MCT for a 2-dimensional state vector. 


\subsection{Performance measures}

This section introduces a number of measures to evaluate the performance of a state estimator. The Cramér-Rao lower bound (CRLB) provides a theoretical bound on the performance of a specific model and sensor setup, while the loglikelihood metric indicates how well a specific system of models represents a particular dataset.

\subsubsection{Cramér-Rao lower bound}

The CRLB provides a lower bound of the variance of an unbiased estimator [52], and highlights the impossibility of finding an unbiased estimator with a variance less than the bound. This provides a benchmark against which the performance of an available estimator can be compared. Given the regularity condition

$$
\mathbf{E}_{\mathbf{y} \mid \mathbf{x}}\left[\nabla_{\mathbf{x}} \log p(\mathbf{y} \mid \mathbf{x})\right]=0 \quad \text { for all } \mathbf{x},
$$

where $\nabla_{\mathbf{x}}$ denotes the gradient operator with respect to the state vector, and the expected value is taken with respect to $p(\mathbf{y} \mid \mathbf{x})$, which is the probability function of the observations $\mathbf{y}$ with the state $\mathbf{x}$ as a parameter. The CRLB gives a bound on the variance of any unbiased estimator of $\mathbf{x}$

$$
\operatorname{cov}(\hat{\mathbf{x}}) \geq \mathcal{I}^{-1}\left(\mathbf{x}^{0}\right),
$$

where $\geq$ is interpreted as $\left[\operatorname{cov}(\hat{\mathbf{x}})-\mathcal{I}^{-1}\left(\mathbf{x}^{0}\right)\right]$ being a positive semi-definite matrix, and $\mathcal{I}\left(\mathbf{x}^{0}\right)$ denotes the Fisher information matrix (FIM) evaluated in the true state $\mathbf{x}^{0}$. The FIM is defined by

$$
\mathcal{I}\left(\mathbf{x}^{0}\right)=-\mathbf{E}_{\mathbf{y} \mid \mathbf{x}}\left[\left.\nabla_{\mathbf{x}}^{2} \log p(\mathbf{y} \mid \mathbf{x})\right|_{\mathbf{x}=\mathbf{x}^{0}}\right],
$$

where $\nabla_{\mathbf{x}}^{2}$ denotes the Hessian with respect to the state, and the derivatives are evaluated in $\mathbf{x}=\mathbf{x}^{0}$.

Information is additive [32] and using the datasets $\mathbf{y}_{1: N}$ of independent observations $\mathbf{y}_{i}$ to estimate $\mathbf{x}$, each $\mathbf{y}_{i}$ having the information $\mathcal{I}_{i}(\mathbf{x})$, yields

$$
\mathcal{I}\left(\mathbf{x}^{0}\right)=\sum_{i=1}^{N} \mathcal{I}_{i}\left(\mathbf{x}^{0}\right) .
$$

For the special case of a Gaussian distribution of the observations

$$
\mathbf{y} \sim \mathcal{N}\left(\mu\left(\mathbf{x}^{0}\right), \mathbf{P}\left(\mathbf{x}^{0}\right)\right)
$$

the FIM is given by [52]

$$
\begin{aligned}
{\left[\mathcal{I}\left(\mathbf{x}^{0}\right)\right]_{i j}=\left(\nabla_{\mathbf{x}_{i}} \mu\left(\mathbf{x}^{0}\right)\right)^{\top} \mathbf{P}^{-1}\left(\mathbf{x}^{0}\right) } & \left(\nabla_{\mathbf{x}_{j}} \mu\left(\mathbf{x}^{0}\right)\right) \\
+ & \frac{1}{2} \operatorname{tr}\left[\mathbf{P}^{-1}\left(\mathbf{x}^{0}\right)\left(\nabla_{\mathbf{x}_{i}} \mathbf{P}\left(\mathbf{x}^{0}\right)\right) \mathbf{P}^{-1}\left(\mathbf{x}^{0}\right)\left(\nabla_{\mathbf{x}_{j}} \mathbf{P}\left(\mathbf{x}^{0}\right)\right)\right],
\end{aligned}
$$


where $[\cdot]_{i j}$ denotes the $(i, j)$-element of the matrix and $\mathbf{x}_{i}$ denotes the $i$ th element of the state vector. A special case of (2.26) used in Chapter 4 is when the covariance is independent of $\mathbf{x}$ and the second term evaluates to zero. The information matrix can then be written in matrix form as

$$
\mathcal{I}\left(\mathbf{x}^{0}\right)=\nabla_{\mathbf{x}}^{\top} \mu\left(\mathbf{x}^{0}\right) \mathbf{P}^{-1} \nabla_{\mathbf{x}} \mu\left(\mathbf{x}^{0}\right) .
$$

\subsubsection{Information measures}

When a filter is used for state estimation, a measure based on the covariance or information matrix can be used as an indicator of the performance of the estimator. To allow for comparison between different estimators a scalar measure is desirable. A comprehensive analysis of several alternatives, all based on the eigenvalues of the covariance- or information matrix, is performed in [117]. Here, only the most common alternatives are briefly introduced. For all of the measures it is desirable to minimize the value for the covariance matrix and maximize the value for the information matrix.

\section{Trace}

The trace of the covariance or information matrix is the same as the sum of its eigenvalues

$$
\operatorname{tr}(\mathbf{A})=\sum \operatorname{eig}(\mathbf{A})
$$

Under a Gaussian assumption a covariance matrix can be represented as an ellipsoid bounding a likely value of the state vector given a specified probability. The length of the principal axes of the ellipsoid is proportional to the eigenvalues of the covariance matrix. The trace of the covariance matrix then represents the sum of the length of those axes, and can be interpreted as the overall mean square error (MSE).

\section{Determinant}

The determinant of a matrix is the product of its eigenvalues,

$$
\operatorname{det}(\mathbf{A})=\prod \operatorname{eig}(\mathbf{A})
$$

As with the trace, the determinant has an interpretation for a covariance matrix. The determinant is proportional to the volume of the ellipsoid determined by the covariance matrix.

\section{2-norm}

The 2-norm of a covariance- or information matrix equals its largest eigenvalue

$$
\|\mathbf{A}\|_{2}=\max [\operatorname{eig}(\mathbf{A})] \text {. }
$$

For the covariance matrix, this corresponds to the length of the major axis of the associated ellipsoid. 


\subsubsection{Log-likelihood}

The CRLB, and the information measures, give insight to how well a state estimator can possibly perform under the conditions of a specific sensor setup and model, assuming that the estimator is unbiased. However, in cases where the full structure of the model is not known, or in real life application where the model is an approximation of a physical system and the estimator is most certainly not unbiased, some other metric is needed. To evaluate how well a model represents a specific dataset, the log-likelihood of the estimate is often used as a performance measure.

If the true state $\mathbf{x}^{0}$ of a system is known, often referred to as the ground truth, the likelihood of an estimator to actually estimate the true value of the state vector are the probability of $\hat{\mathbf{x}}=\mathbf{x}^{0}$. If the state estimate has a Gaussian distribution, this probability can be written as

$$
p\left(\hat{\mathbf{x}} \mid \mathbf{x}^{0}\right)=\frac{1}{(2 \pi)^{\frac{n_{x}}{2}}(\operatorname{det} \hat{\mathbf{P}})^{\frac{1}{2}}} e^{-\frac{1}{2}\left(\hat{\mathbf{x}}-\mathbf{x}^{0}\right)^{\top} \hat{\mathbf{P}}^{-1}\left(\hat{\mathbf{x}}-\mathbf{x}^{0}\right)},
$$

where $n_{x}$ is the dimension of the state vector. The natural logarithm is a monotonically increasing function, and the logarithm of a function has the same extreme points as the function. It also has the benefit of simplifying computations in this case and therefore the logarithm of the probability density function (PDF) is often considered [52]

$$
\log p\left(\hat{\mathbf{x}} \mid \mathbf{x}^{0}\right)=-\frac{n_{x}}{2} \log (2 \pi)-\frac{1}{2} \log (\operatorname{det} \hat{\mathbf{P}})-\frac{1}{2}\left(\hat{\mathbf{x}}-\mathbf{x}^{0}\right)^{\top} \hat{\mathbf{P}}^{-1}\left(\hat{\mathbf{x}}-\mathbf{x}^{0}\right) .
$$

If no ground truth for the state vector is available for evaluation, the observations can be used instead. The likelihood of an observation conditioned on the state estimate $p(\mathbf{y} \mid \hat{\mathbf{x}})$ is then considered. For a Gaussian distribution of $\mathbf{y}$ this can be written as

$$
\log p(\mathbf{y} \mid \hat{\mathbf{x}})=-\frac{n_{y}}{2} \log (2 \pi)-\frac{1}{2} \log (\operatorname{det} \hat{\mathbf{S}})-\frac{1}{2}(\mathbf{y}-\mathbf{h}(\hat{\mathbf{x}}))^{\top} \hat{\mathbf{S}}^{-1}(\mathbf{y}-\mathbf{h}(\hat{\mathbf{x}})),
$$

where $\mathbf{h}(\hat{\mathbf{x}})$ predicts the observations given the estimated state $\hat{\mathbf{x}}$, and $\hat{\mathbf{S}}$ is the covariance of these expected observations. The log-likelihood considers both the mean and covariance of the state estimate and provides the maximum likelihood estimator if used as cost function in an optimization problem. This approach is used in Chapter 3.

Note that the last term in (2.32) and (2.33) are often referred to as the normalized estimated error squared (NEES) and normalized innovation squared (NIS), respectively. The NEES and NIS can be used for consistency tests or divergence monitoring of a filter implementation [20,32].

\subsection{D LIDAR sensors}

In robotics, $2 \mathrm{D}$ LIDAR is a popular choice of sensor for $2 \mathrm{D}$ localization applications. A LIDAR measures the distance, along beams with different transmission 
angles, to nearby objects or walls. This section considers the properties of 2D LIDAR sensors that are important in a state estimation setting. The LIDAR data is utilized in the measurement update in the filter framework, and in this section different measurement models with associated noise properties are discussed. Figure 2.2 depicts typical 2D LIDAR scans from commonly used and freely available datasets, one collected in an office environment and another in an outdoor park.

\subsubsection{Ray measurement model}

A LIDAR sensor typically outputs a complete scan of measurements, covering all transmission angles. However, an intuitive model for LIDAR observations is obtained by first considering only one ray measurement. For ray $i$ in a scan containing $m$ rays, the measurement function can be written as,

$$
y_{i}=r_{i}+e_{i}, \quad i=1, \ldots, m,
$$

where $r_{i}$ is the measured range from the sensor to a point in the environment that this particular ray hits, and $e_{i}$ is noise. The range depends on the state and on the surroundings. Let $\mathcal{M}$ denote a representation of the surrounding, e.g., a map of the operation area. Individual ray measurements from a complete scan are then stacked in a vector as $\mathbf{y}=\left[\begin{array}{llll}y_{1} & y_{2} & \ldots & y_{m}\end{array}\right]^{\top}$. For a given time step, the measurement function in (2.2) can be written as,

$$
\mathbf{y}_{k}=\mathbf{h}\left(\mathbf{x}_{k}, \mathcal{M}\right)=\mathbf{r}_{k}+\mathbf{e}_{k},
$$

where $\mathbf{r}_{k}$, and $\mathbf{e}_{k}$ are similarly stacked vectors with ranges and noises, respectively. When this measurement model is used in a filter, $\mathbf{h}\left(\mathbf{x}_{k}, \mathcal{M}\right)$ is often computed using ray-casting, which is a non-trival and computationally expensive operation. A sensor is virtually placed in the environment and each ray trajectory is traversed until it hits a wall or some other object in the map.

\section{Simplistic noise model}

A simplistic noise model is obtained by assuming white Gaussian noise, $\mathbf{e}_{k}$ $\mathcal{N}\left(\mathbf{0}, \mathbf{R}_{k}\right)$ [103]. The covariance $\mathbf{R}_{k}$ is in the simplest case diagonal, by assuming each ray measurement being independent of one another. Often also the same variance is assumed for each ray and each time step, $\mathbf{R}_{k}=\sigma_{R}^{2} \mathbf{I}$. In the probabilistic framework, this yields,

$$
p(\mathbf{y} \mid \mathbf{x}, \mathcal{M})=\mathcal{N}\left(\mathbf{y} \mid \mathbf{r}, \sigma_{R}^{2} \mathbf{I}\right) .
$$

Though heavily simplified, this model is often used with good results by increasing the magnitude of $\sigma_{R}^{2}$ to incorporate all possible error sources $[32,54]$.

\section{Physical noise model}

By taking a closer look on the physics of a laser beam, it is evident that the assumption that the variance is equal for all rays is not valid. The laser beam is in fact cone-shaped and the variance of the measurement depends on the range 


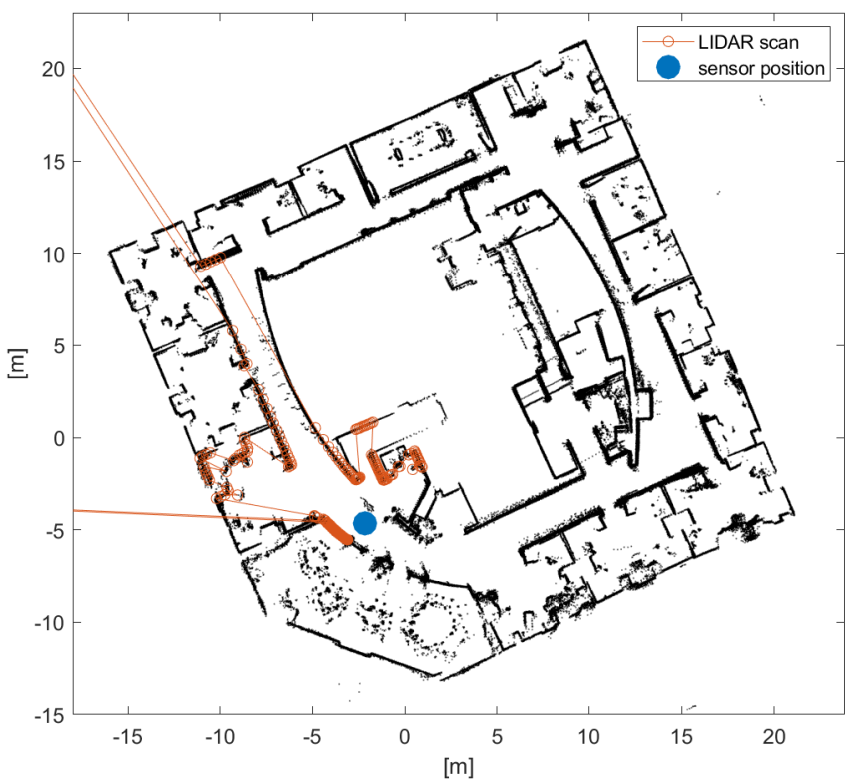

(a) The Intel dataset is freely available in the Radish repository and the github repository accompanying [107] provides the ground truth data used to obtain this picture.

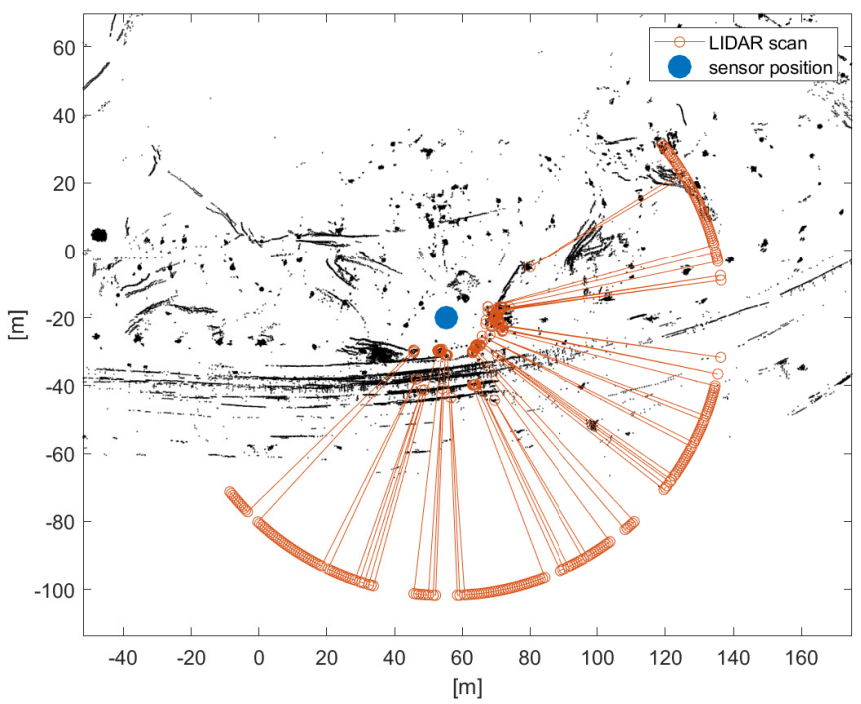

(b) The Victoria park dataset, available through [30] and the ground truth data used to generate this picture is provided in the github repository accompanying [107].

Figure 2.2: Examples of point clouds acquired by 2D LIDAR's in an indoor office environment and an outdoor park. Blue circles mark sensor positions. 


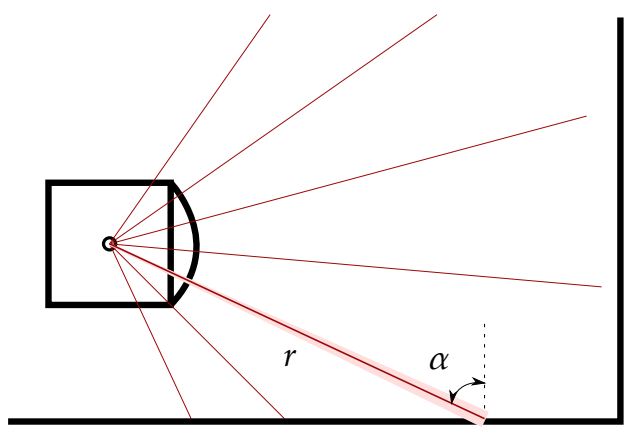

Figure 2.3: A laser beam is in fact cone shaped. This induces errors in the measurement dependent on the nominal measured range $r$, and the angle of incidence $\alpha$, to the measured target.

of the measurement and on the angle of incidence of the ray to the object it hits [79], see Figure 2.3. If $\mathbf{R}_{k}=\operatorname{diag}\left(\sigma_{1}^{2}, \sigma_{2}^{2}, \ldots, \sigma_{m}^{2}\right)$ the variance for a single ray is proportional to the squared range, and inversely proportional to the cosine of the inclination angle,

$$
\sigma_{i}^{2} \propto \frac{\lambda r_{i}^{2}}{\rho \cos \alpha_{i}} \propto \frac{r_{i}^{2}}{\cos \alpha_{i}},
$$

where $\lambda$ is the wavelength of the emitted laser and $\rho$ is the reflectance of the target object. By using the range and inclination angle, a better approximation of the variance, individual to each ray, can be used in the model.

\section{Error sources}

The error modeled by the variance in (2.37) is due to the limited resolution of the sensor and other physical effects of the laser beam. However, in a 2D position estimation setting there are other sources where possibly large errors can originate from.

- Unexpected objects. Objects not presented in the map, causes the LIDAR to produce unexpectedly short range measurements. Typical objects are other vehicles, humans passing by, mist, fog, and water particles in the air, or something else in the environment that has been changed since the map was produced. These objects cause range measurements shorter than expected, never longer, and will therefore introduce a bias in the measurements.

- No return measurements. Sometimes, objects or wall sections are totally unseen by the LIDAR ray, causing a max-range measurement output from the LIDAR. In many circumstances this is a quite frequent event, especially when the reflectance of the target is low. No return measurements are also obtained if the sensor for some reason does not register the reflected ray.

- Errors in the map. If the map is not a perfect representation of the operation area, a ray-casting algorithm will produce expected measurements 
with potentially large differences to real observations.

- Incorrect planar 2D world assumption. If the sensor is not completely horizontally mounted, or if the ground where the robot is moving is not perfectly planar, roll and pitch of the vehicle causes the laser ray to hit a different vertical spot on the object than intended. This adds errors especially for long range measurements, and the error is even larger if the environment is not constant in the vertical direction.

- Random measurements. Finally, sensors occasionally produce entirely unexplainable measurements. This could be from sources not included in this list or phantom readings subject to unexpected multi-path effects or crosstalk between different sensors.

\section{Advanced error models}

To better describe the error sources presented above a noise model more sophisticated than independent and identically distributed (iid) Gaussian noise is suitable. In [104] a probabilistic approach is taken where all possible error sources are modeled as a stochastic distribution, not necessarily Gaussian. The distributions are linearly combined to form a distribution for the total measurement error. Intrinsic parameters can then be learned for a specific sensor and operation environment.

The concept of learning parameters for a specific setup is also explored in [103], where a model originally developed to simulate realistic LIDAR data is presented. An additive Gaussian noise model is assumed as in (2.34) where each ray is considered separately. The mean and covariance of $e_{i}$ is then assumed to depend on the range and inclination angle of the measurement,

$$
e_{i} \sim \mathcal{N}\left(\mu_{e}\left(r_{i}, \alpha_{i}\right), \sigma_{e}^{2}\left(r_{i}, \alpha_{i}\right)\right)
$$

The probability of obtaining a no-return measurement $P_{\text {noreturn }}\left(r_{i}, \alpha_{i}\right)$ is also introduced in the measurement model, which yields

$$
y_{i}= \begin{cases}\max \text { range, } & \text { with probability } P_{\text {noreturn }}\left(r_{i}, \alpha_{i}\right) \\ r_{i}+e_{i}, & \text { with probability }\left(1-P_{\text {noreturn }}\left(r_{i}, \alpha_{i}\right)\right)\end{cases}
$$

for one ray. The functions $\mu_{e}\left(r_{i}, \alpha_{i}\right), \sigma_{e}^{2}\left(r_{i}, \alpha_{i}\right)$ and $P_{\text {noreturn }}\left(r_{i}, \alpha_{i}\right)$ can be learned from data recorded with a specific setup, resulting in a realistic error model for this particular scenario. Various regression techniques can be used for learning [103]. In this thesis a support vector machine (SVM) model [13] is used to simulate realistic laser data.

\subsection{Scan registration}

Instead of considering each ray in a laser scan individually in the measurement model, as in (2.35), there are methods to process a complete scan at once. This 
section considers scan registration methods and how they can be incorporated in the filtering framework.

A scan registration method matches and aligns two scans, where the output often is a rigid body transformation $(R, t)$, between the scans. A well established algorithm worth introducing is the iterative closest point (ICP) method $[10,19]$. The ICP algorithm alternates between nearest neighbor association and least-squares optimization to compute the best rigid body transformation between two point clouds given the most recent association.

Given two point clouds

$$
\begin{aligned}
& \mathcal{P}_{k}=\left\{\mathbf{p}_{i}^{k}\right\}_{i=1}^{N_{k}}, \\
& \mathcal{P}_{l}=\left\{\mathbf{p}_{i}^{l}\right\}_{i=1}^{N_{l}},
\end{aligned}
$$

with $\mathbf{p}_{i} \in \mathbb{R}^{D}$, the following optimization problem is solved

$$
\min _{R, t} \mathbf{C}\left(\mathcal{P}_{k}, \mathcal{P}_{l}\right)=\min _{R, t} \sum_{i=1}^{N_{k}} \sum_{j=i}^{N_{l}} w_{i, j}\left\|\mathbf{p}_{i}^{k}-\left(R \mathbf{p}_{j}^{l}+t\right)\right\|^{2},
$$

where $w_{i, j}$ is 1 if point $\mathbf{p}_{i}^{k}$ and point $\mathbf{p}_{j}^{l}$ are assumed to describe the same point in space, and 0 otherwise. The algorithm iteratively finds the nearest neighbor point pair, then computes $(R, t)$ and repeats until convergence [29].

The ICP algorithm is fast and in general produces good results when the initial offset between the scans is small [86]. The original version of the algorithm provides no estimate of the uncertainty of the resulting transform, but various versions have been developed over the years to improve performance and specializations for specific applications [9, 14, 27, 68, 83, 95]. In [69] ICP is used for underground navigation.

An alternative to ICP for scan registration is the normal distribution transform (NDT) [11] where the 2D plane is divided into cells and each cell is assigned a normal distribution modeling the probability of measuring a point.

In [86] a conditional random field (CRF) [58] is used to match 2D laser scans. Some locally defined features computed for each laser point (for example, relative distances between points in the scan, angle between the segments connecting a point to its neighbors or sum of distances to neighboring points), are combined to define a signature for a complete scan. This signature is then used to produce a rigid body transformation between the scans.

The transformation obtained from a scan registration method, can be converted to an indirect measurement of the state vector. This reduces the measurement model to,

$$
\mathbf{y}_{k}=\mathbf{x}_{k}+\mathbf{e}_{k}
$$

causing the complete filter to act as a low pass filter for the state estimate. This measurement model gives an enormous reduction of computational complexity in the measurement update of the filter since it reduces the dimensionality of the measurement space. Inference in a high-dimensional measurement space is 
costly and inference in the low-dimensional space can be orders of magnitude more efficient [104]. That is, as long as the scan registration algorithm itself is computationally feasible.

\subsection{Feature extraction}

The measurement models (2.35) and (2.42) in Section 2.3 and 2.4, respectively, are based on raw sensor measurements. An alternative approach is explored in this section where the data is preprocessed and features are extracted from the measurements. Feature extraction methods are available for different sensor types, but the focus in this thesis is on feature extraction for 2D LIDAR data.

As for the measurement model in (2.42) emerging from a scan registration method, a benefit from pre-processing the raw sensor data and extract high-level features is the reduced dimensionality of the observation space. Most feature extraction methods extract a small number of features from high dimensional LIDAR measurements [104]. As discussed in Section 2.4, this reduces the computational complexity in the filter algorithm. Furthermore, a feature extraction approach makes it possible to perform a filter update without the computationally costly ray-casting operation. As further investigated in Chapter 5, this approach also increases robustness against errors in the map.

The computer vision community has successfully built an arsenal of feature extraction methods for the purpose of getting robust data associations in noisy data with low computational effort [56]. Procedures developed for image and point cloud processing have also been adopted for feature extraction methods specifically adapted to 2D LIDAR data [50,107, 110]. These 2D LIDAR feature extraction methods are proven to perform well in clean indoor environments. The established procedure to recognize points, regions, or objects in sensor data consists of first detecting interest points, also known as keypoints, and then computing a distinctive signature for each of them, called a descriptor. A keypoint is ideally viewpoint invariant, repeatable and descriptive over the region of detection. The descriptor is usually a vector encoding the (physical) neighborhood of the keypoint enabling more robust matching among points acquired from different viewpoints, with different sensor noise, and which might also be affected by occlusion. Incorporating the feature extraction procedure into the positioning problem gives the work-flow of a measurement update, as depicted in Figure 2.4, starting with the input sensor data, resulting in a position estimate of the moving vehicle.

Detectors and descriptors are typically presented in pairs as complete feature extraction methods. However, it is in general possible (but not always computationally efficient) to use any detector in combination with any arbitrarily chosen descriptor. Therefore, the detectors and descriptors are here presented separately. 


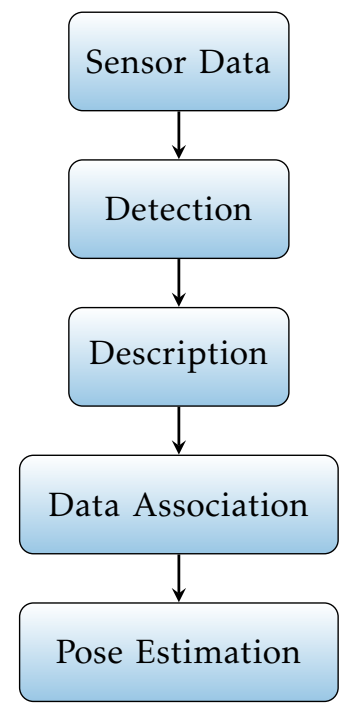

Figure 2.4: The work-flow when a feature extraction method is used for positioning. A detector searches the input sensor data for keypoints, and a descriptor computes a distinctive signature for each of them. The descriptors are used in the data association step to enable more robust correspondence matching when data is acquired from different viewpoints.

\subsubsection{Sensor data}

Data from 2D LIDAR sensors can easily be converted to points clouds. Often when a point cloud is considered it is assumed to be a, compact, dense, and in many cases, 3D point cloud, see Figure 2.5 for an example. A point cloud produced by a 2D LIDAR contains much less information and is very sparse in comparison to a $3 \mathrm{D}$ point cloud. The point cloud is, at least approximately, describing a $2 \mathrm{D}$ plane, and depending on the environment the data is more or less scattered, see Figure 2.2.

\subsubsection{Detectors}

A substantial amount of research effort is put into object detection and recognition in images. For 3D point clouds, several keypoint feature detectors have been proposed that mainly originate, or at least are strongly influenced by, methods from image processing, see for example $[7,67,94,109]$. Since this type of data is not the focus of this thesis, an interested reader is referred to some of the many surveys and performance evaluations available on this topic [28, 31, 96]. From now on, only 2D point clouds are considered. 


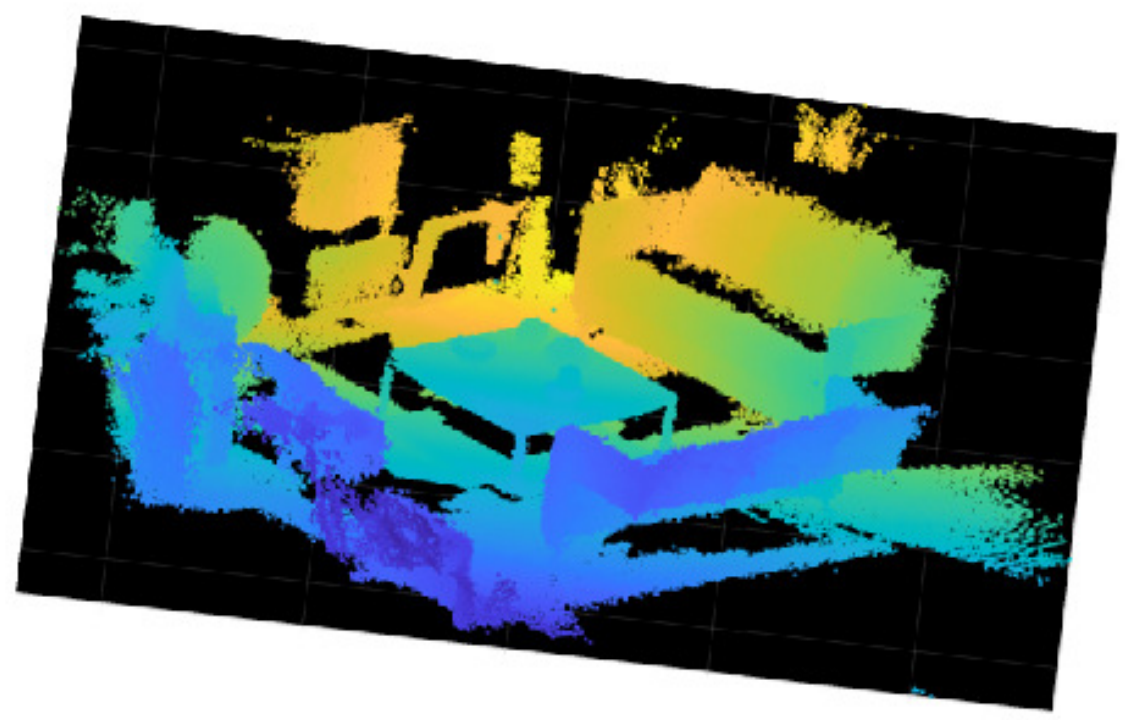

Figure 2.5: $3 D$ point cloud produced by a RGB-D (Kinect-style) camera, showing a scene containing furniture [59]. The colors indicate distance from the sensor.

\section{Image feature detectors}

By converting 2D laser data into an image, feature detectors designed for images can be directly applied without further adaptions. Detailed descriptions of different algorithms for image feature detection can be found in [56]. Some worth mentioned by name are:

- The Harris corner detector [36], which defines a corner as the crossing of two different edge directions, where an edge is a sudden change in image intensity. A structure tensor is computed consisting of the covariance of directional derivatives in the local neighborhood of a pixel. From the eigenvalues of this tensor it can be identified if the pixel is uninteresting, on an edge or if it represents a corner point.

- The Shi-Tomasi detector [100], is an optimization of the Harris corner detection. By only considering the minimum eigenvalue, the computational complexity is considerably reduced.

- The scale-invariant feature transform (SIFT) [66], convolves a raw image by Gaussian kernels at different scales, $t$, to form blurred or smoothed images. 
Differences of successive smoothed images at different scales are formed, and keypoints are taken as extreme points of the difference images.

In $[62,63], 2 \mathrm{D}$ LIDAR data is rasterized into an image and Shi-Tomasi corner detection is applied. This approach enables the re-use of elaborate computer vision algorithms. However, it is computationally expensive to perform rasterization of laser measurements and the rasterization introduces inaccuracies.

\section{D LIDAR detectors}

Data acquired from a 2D LIDAR consists of relatively few points, the point density is highly non-uniform and not view-point invariant. The nature of the data affects how to extract stable and distinguishable keypoints, and there are few works specialized on 2D laser data keypoint features.

One of the first attempts to detect and describe keypoints in $2 \mathrm{D}$ range data is [14], where keypoints are selected at locations of high curvature in submaps defined as a collection of multiple laser scans. In the subsequent work [15], different types of keypoint detectors are tested, but all of them search for keypoints in a submap rather than in a single scan. This makes their approach more of a submap characterization technique for place recognition rather than a feature extraction method.

Although, general feature detectors specialized on 2D range data are few, there exist three quite recently developed such methods presented below, namely fast laser interest region transform (FLIRT), fast adaptive laser keypoint orientation invariant (FALKO), and B-spline based interest point detector (BID).

\section{FLIRT}

In [107] the fast laser interest region transform (FLIRT) is presented as a detector for locally defined keypoints for 2D laser data. The FLIRT method adopts the scale space theory used in SIFT. First, the original data is represented by a family of smoothed signals parameterized by $t$, the size of a Gaussian smoothing kernel. Then, differences of the smoothed signals are formed and peaks are identified to locate keypoints. Three different detectors are presented in [107]. The first one operates on the raw range data and the second one on a local approximation of the normal direction in each point. The third, and also the best performing one according to simulations performed in the paper, adapts the work done for $3 \mathrm{D}$ point clouds in [109] to $2 \mathrm{D}$ range data. The range data defines a curve in Cartesian 2D space and the scale space theory is applied to this curve. An integral operator maps the input curve into a multi-scale parameterization

$$
S(\alpha(s) ; t)=\int_{\Gamma} k(s, u ; t) \alpha(u) d u,
$$

where $\Gamma$ is the curve, $\alpha(s)$ is the parameterization of the curve by the geodesic coordinate $s$, and $k(s, u ; t)$ is a Gaussian kernel with mean $s-u$ and standard deviation $t$. The integral is approximated by a sum and to compute the Gaussian 
kernels in the sum the geodesic distance between each observed point pair is required. As suggested in [109], disjoint minimum spanning trees (DMST) are used for this purpose in the FLIRT algorithm, where the number of disjoint trees is a design parameter. When DMST is used for the curvature approximations, the range data is considered an unordered point cloud, and extreme outliers such as no-return data are automatically ignored, see [17] for details.

Keypoints are then detected as local maxima of the exponential damping expression

$$
F\left(\mathbf{p}_{i}, t\right)=\frac{2\left\|\mathbf{p}_{i}-S(\alpha(s), t)\right\|}{t} e^{-\frac{2\left\|\mathbf{p}_{i}-S(\alpha(s), t)\right\|}{t}},
$$

where $\mathbf{p}_{i}$ represents each scan point. To find peaks of $F\left(\mathbf{p}_{i}, t\right)$ two design parameters have to be determined, the minimum value of $F\left(\mathbf{p}_{i}, t\right)$ to be considered a peak $\left(F_{\min }\right)$ and the minimum separation between peaks $\left(F_{\text {dist }}\right)$.

The scales to consider for the curve fitting are determined by

$$
t=t_{0}\left(t_{i}\right)^{k}
$$

where $t_{0}$ and $t_{i}$ are scalar design parameters, $k \in 0,1, \ldots n_{k}-1$ is the current scale, and $n_{k}$ gives the number of scales.

\section{FALKO}

In [50] a novel keypoint detector specialized on 2D range data is proposed, the fast adaptive laser keypoint orientation-invariant (FALKO) detector. This method focuses on finding stable view-point invariant keypoints like corners, rather than gaps and isolated points. The detector is designed to be orientation invariant and measurement sparsity independent by considering neighboring data points within a variable radius of the candidate point. A set of neighboring points of an observed point $p_{i}$, is defined as

$$
C\left(\mathbf{p}_{i}\right)=\left\{\mathbf{p}_{j} \in \bar{S}:\left\|\mathbf{p}_{j}-\mathbf{p}_{i}\right\|<r_{i}\right\},
$$

where the radius $r_{i}$ is computed as

$$
r_{i}=a e^{b\left\|\mathbf{p}_{i}\right\|},
$$

where $\left\|\mathbf{p}_{i}\right\|$ is the distance from the observed point to the sensor origin. The design parameters $a$ and $b$ scale the neighborhood radius, where $a$ has most effect on short range measurements, whereas $b$ has a greater impact on longer ranges. The two endpoints in each neighbor set $\mathbf{p}_{j_{\min }}$ and $\mathbf{p}_{j_{\max }}$ do, together with the point $\mathbf{p}_{i}$ itself, form a triangle. As a first rough approximation of a corner, this triangle is used to discard many points as keypoints candidates. If the base or height of the triangle is less than $\frac{r_{i}}{\beta}$ the point is discarded. Greater values of $\beta$ allows both wider and sharper corners as candidate points.

For the remaining candidate points, the set $C\left(\mathbf{p}_{i}\right)$ is divided into two subsets

$$
\begin{aligned}
& C_{L}\left(\mathbf{p}_{i}\right)=\left\{\mathbf{p}_{j} \in C\left(\mathbf{p}_{i}\right): j<i\right\}, \\
& C_{R}\left(\mathbf{p}_{i}\right)=\left\{\mathbf{p}_{j} \in C\left(\mathbf{p}_{i}\right): j>i\right\} .
\end{aligned}
$$


For each point in any of the sets, a quantized orientation $\phi_{j}$, with respect to the candidate point is computed by forming a grid with $s_{n}$ circular sectors centered on the candidate point. The orientation is then defined as in which circular sector the point $\mathbf{p}_{j}$ lays in, and a discrete distance function $d_{\theta_{j}, \theta_{k}}$ is defined as how many sectors are between $\theta_{j}$ and $\theta_{k}$. A cornerness score is then computed for each of the two subsets in (2.48) as the sum of all point pair distances in the subset

$$
\begin{aligned}
& \operatorname{score}_{L}\left(\mathbf{p}_{i}\right)=\sum_{h=i-1}^{j_{\min }} \sum_{k=h-1}^{j_{\min }}\left|d_{\theta}\left(\phi_{h}, \phi_{k}\right)\right|, \\
& \operatorname{score}_{R}\left(\mathbf{p}_{i}\right)=\sum_{h=i+1}^{j_{\max }} \sum_{k=h+1}^{j_{\max }}\left|d_{\theta}\left(\phi_{h}, \phi_{k}\right)\right| .
\end{aligned}
$$

The score is small if the points in the subset is aligned with each other. A total score for a candidate point is then defined as the sum of $\operatorname{score}_{L}\left(\mathbf{p}_{i}\right)$ and $\operatorname{score}_{R}\left(\mathbf{p}_{i}\right)$ and keypoints are chosen as local minima of the scores. To avoid ambiguous keypoints a non-maxima suppression (NMS) procedure is applied defining a minimum distance between keypoints, $\mathrm{NMS}_{\min }$.

\section{BID}

The B-spline based interest point detector (BID) is introduced in [110]. It uses the same concept as FALKO by extracting high curvature candidate points using a set of neighbor points defined as in (2.46) with a radius growing with measured range as in (2.47). The points in $C\left(\mathbf{p}_{i}\right)$ are used to approximate a B-spline curve that in turn is used to determine corner existence. First a B-spline curve is constructed with four control points, evenly distributed along the axis corresponding to the largest eigenvalue of the covariance matrix of $C\left(\mathbf{p}_{i}\right)$. Then, the iterative point distance minimization (PDM) algorithm is applied to the initial $\mathrm{B}$ spline to fit it to the data points in $C\left(\mathbf{p}_{i}\right)$. The resulting B-spline is denoted $S(t)$. The normalized Euclidian distance between two median control points $\mathbf{p}_{1}^{c}$ and $\mathbf{p}_{2}^{c}$ of the approximated curve $S(t)$

$$
E=\frac{\left\|\mathbf{p}_{1}^{c}-\mathbf{p}_{2}^{c}\right\|}{\sum_{i=0}^{2}\left\|\mathbf{p}_{i}^{c}-\mathbf{p}_{i+1}^{c}\right\|},
$$

is used as a measure of curvature. Keypoints are defined as local maxima of the inverse of the normalized Euclidean distance $E^{-1}$ with scores above a threshold $T_{\text {th. }}$.

An advantage with the BID method is that the approximated curve can be interpolated along the B-spline to get a more precise location of the keypoint, that also depends less on the point density. A disadvantage is the computational effort it takes to approximate the B-spline curve. This computational burden is somewhat lowered by a threshold when PDM is applied. If the error, according to (2.50), of the initial curve, is lower than a threshold $E_{\mathrm{th}}$, the PDM is not applied and the candidate point is disregarded. Such situation occurs in low curvature 
regions. As for the FALKO detector a NMS procedure is suggested to avoid ambiguous keypoints.

\section{Computation time comparison}

The FALKO method includes only simple computations and is therefore well suited for real-time applications. This is experimentally verified in [110], where computation times for FLIRT and FALKO are compared to their own contribution BID. FALKO is roughly 50 times faster than FLIRT and 100 times faster than BID.

\section{Geometric detectors}

The extraction of various high-level geometric features have been used in association with LIDAR sensors, e.g., line segments [76, 84, 91, 101], poly-lines [114], curve segments [80], circles [113, 122], Bezier curves [72] or B-splines [82]. These are strictly not feature keypoints as they are not view-point invariant points of interest with an associated descriptor. Due to the use of specific geometric features, these approaches do not provide robust solutions in a general environment of any shape.

\subsubsection{Descriptors}

A good descriptor should capture the signature of a keypoint regardless of viewpoint, sensor noise, or occlusions. When used for localization or place recognition, the detector is usually defined as a fixed-length vector or a histogram, accompanied by a distance metric. This is to make the descriptor computationally efficient when used to robustify the data association step. Although, for image processing there exist more complex descriptors. Two examples are the basis space descriptors, where the descriptor is defined in another basis, and the polygon shape descriptors, where the features are based on the perimeter of a polygon shape [56].

Since the same feature can be perceived from different view-angles, descriptors can also include additional information about the orientation of the keypoint. Thus, for matching, the descriptors can be rotated to be aligned before the distance metric is evaluated.

As for detectors, there has been substantial work performed in the computer vision community $[16,56,61]$, and in [63] the SIFT descriptor is used for $2 \mathrm{D}$ range data. However, the results in $[50,110]$ indicate that stable detection of feature points is much more important than a sophisticated description to obtain good feature matching in $2 \mathrm{D}$ laser data. Therefore the main focus of the reminder of this section is on the descriptors accompanying the FLIRT, FALKO, and BID detectors in [107], [50], and [110], respectively, all originating from the same concept of a shape context. 


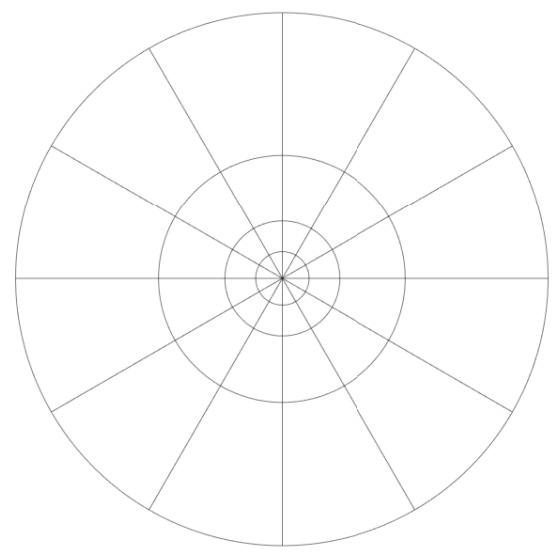

(a) Log-polar histogram

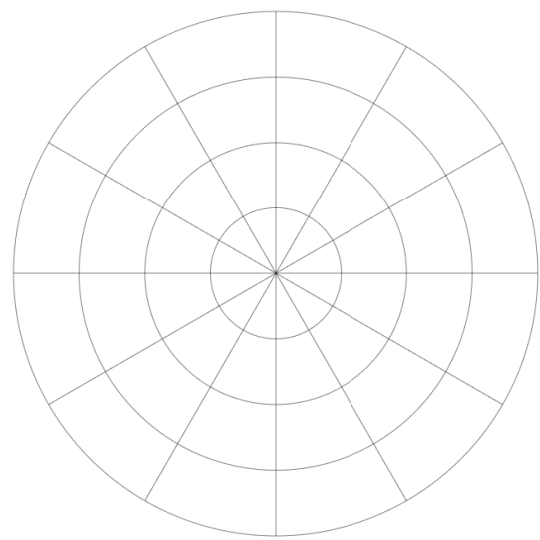

(b) Linear-polar histogram

Figure 2.6: Histograms used by a shape context descriptor.

\section{Shape context}

In the early range data feature extraction attempt in [15], multiple detectors are discussed and evaluated. They consider a region with a fixed radius of $9 \mathrm{~m}$ and include data from multiple scans when computing description vectors. This is suitable for their place recognition purpose, but it cannot really be viewed as a local keypoint feature descriptor. Nevertheless, one of the best performing descriptors in the experiments conducted in [15] is based on the concept of shape contexts, which is also the case for the descriptors used in state-of-the-art range data feature extraction work $[50,107,110]$. The concept of shape contexts is developed for object recognition in images in [8]. Objects are treated as a set of points sampled from the internal or external contours of the object. A reference point is chosen and the distribution of the relative coordinates of the remaining points is represented in a histogram. The bins of the histogram are uniform in $\log$-polar space with $r_{n}$ bins in the radial direction and $\alpha_{n}$ bins in the angular direction, see Figure 2.6a. This histogram is defined to be the shape context descriptor. This type of descriptor suits the range data well since the data is already points and detected keypoints are natural choices of reference points.

\section{$\beta$-grid}

The $\beta$-grid descriptor is introduced in [107]. A linear-polar histogram (see Figure $2.6 \mathrm{~b}$ ) is suggested to reduce the impact of measurement noise that typically occurs in radial direction in LIDAR data, and would be captured in the bins near the centre in a log-polar histogram. The radius of the histogram $\rho_{\max }$ as well as an inner threshold $\rho_{\min }$ for when data points are ignored and assumed ambiguous, are design parameters for the $\beta$-grid descriptor, together with $r_{n}$ and $\alpha_{n}$ defined 
in the section above. The symmetric $\chi^{2}$-distance function defined as

$$
d=\frac{1}{2} \sum_{i} \frac{\left(x_{i}-y_{i}\right)^{2}}{\left(x_{i}+y_{i}\right)},
$$

where $x_{i}$ and $y_{i}$ are elements in different histograms, is used to compare descriptors. The $\beta$-grid descriptor also encodes the probabilities of free-space into the histogram by applying Bayesian parameter learning. This gives a distinction between a concave and a convex structure, but it only gives slightly improved performance in experiments, and it could be questioned if the increased computational complexity is worth it.

\section{Binary shape context (BSC)}

The binary shape context (BSC) descriptor accompanying the FALKO detector is introduced in [50]. This descriptor uses the same structure with the linear-polar histogram as in [107], but limits the histogram to be binary. With bins that are either filled or empty, the influence of the point density is reduced. A natural choice of $\rho_{\max }$ is the region for where the set of neighboring points are defined, resulting in different histogram sizes for different keypoints, $\rho_{\max }=r_{i}$. The distance metric associated with this descriptor is a Hamming-like function and the orientation of each keypoint can be computed using a variant of the intensity centroid presented in [92].

\section{Spline distribution histogram (SDH)}

In [110] the similar spline distribution histogram (SDH) descriptor is proposed, but with improvements using results from their B-spline based detector. Since Bspline approximations of the curve in the vicinity of a keypoint is performed in the detection step, the curve can be interpolated to remove the point density dependency. The histogram can be kept non-binary not to lose information about the alignment of neighboring points. The interpolation is done with equal distance $d_{t}$ between points along the spline. The symmetric $\chi^{2}$-distance is used to measure the distance between two SDH descriptors. The orientation of keypoints are computed using the centroids as in [50], but now applied to uniformly sampled points along the approximated spline instead of neighboring points from the point cloud.

\subsubsection{Data association}

In order to use keypoints obtained by feature extraction in a state estimation application, each keypoint in a point cloud has to be associated to a specific keypoint in another point cloud. The purpose of data association is twofold; it should match keypoints detected in different point clouds corresponding to the same actual feature, and it should filter out and ignore outliers not having a true match in any of the point clouds. 


\section{Feature matching}

Even though feature descriptors are designed to provide robust feature matching between scans, pure descriptor association usually gives poor results in experiments $[50,73,107]$. Therefore, some other data association technique is often used in conjunction with the feature descriptors. For position estimation purposes, where a good initial estimate is known, the descriptors can be used as a gating rule for geometric associations. For place recognition and loop closure applications other data association algorithms are often used, e.g., geometrical landmark relations (GLARE) [37, 50, 110], where 2D laser scans are transformed into pose invariant histogram representations, or, as in $[6,110]$, a graph theoretic approach. The relative geometry between points in a scan is used to build graphs and then the maximum common subgraph is used to match different scans. In [49] a comparison of different data association techniques based on extracted FALKO features is performed. The Hungarian algorithm, a combinatorial optimization method for solving assignment problems, is suggested as a data association method well suited for shape context descriptors [50, 56], but no experiments provide any insight of the performance when applied to $2 \mathrm{D}$ laser data.

\section{Outliers}

Data association in noisy data containing outliers can be a serious problem in robust state estimation. When individual matching of laser rays are used, an outlier gives false information as input to the state estimation method. In scan matching algorithms acting on raw data, outliers can cause inaccurate transformations or possibly incorrect matching. Random sample consensus (RANSAC) [25] is an iterative method designed to robustly handle outliers in data. This method is very general and can be applied to various kinds of data. It is a common strategy to increase robustness towards outliers in scan matching algorithms, and in [107] it is used for loop closure detection on keypoint feature data.

Using a feature extraction approach, sporadic outliers can either be filtered out by a sophisticated detector/descriptor (as e.g., the DMST in FLIRT), or it results in keypoints that cannot be matched and therefore ignored for further processing. If lots of outliers are present this can of course result in severe information loss, but a state estimation method is never fed with false information. In addition to this already built in robustness against outliers, RANSAC can of course also be applied to detected keypoints to further improve the outlier rejection capabilities. 


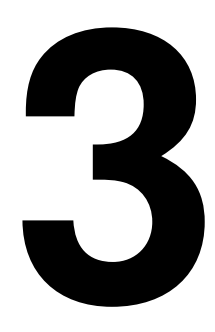

\section{Design parameters in the unscented Kalman filter}

This chapter addresses the problem of proper tuning of the design parameters of the UKF. With bad design parameter values the UKF can have embarrassingly poor performance $[24,108]$. Yet there exists no established procedure for how to tune them. Attempts have been made to perform data driven automatic tuning of the parameters, but without providing intuition of why a parameter setup is particularly good or bad for a certain problem. In [102] the parameter $\kappa$ is tuned with a maximum likelihood estimate, and in [98] the tuning of the design parameters, as well as the choice of process and measurement noise, is formulated as an optimization problem, solved by a stochastic search algorithm. Tuning of the process and measurement noise is also considered in [20], where an approach based on Gaussian processes (GP) is presented. In [108] a GP-based optimizer is used to tune $\alpha, \beta$, and $\kappa$ by learning from sequences of data. This method is computationally intractable for the underground mine application since simulation of trajectories is time consuming and results in a too expensive cost-function. In this chapter a simpler automatic tuning procedure of the design parameters is proposed, still providing good results. The obtained parameter values are also shown to be inline with developed guidelines and heuristics, providing tools to reason about why a particular parameter setup is good or bad for a specific problem.

This chapter starts by unfolding details about the UT and the origin of the associated design parameters in Section 3.1. In Section 3.2 guidelines for choosing parameter values are developed, followed by an optimization based tuning method presented in Section 3.3. Section 3.4 contains verifying experiments with both a simpler simulated example and with real data recorded in an underground mine environment. 


\subsection{Unscented transformation}

The UKF was developed as an approximative solution for nonlinear filtering problems [47]. However, the internally used UT can be interpreted from the perspective of other computational frameworks and taking these viewpoints allow for useful insights.

- In [60] the UT is derived as a special case of statistical linear regression.

- For a Gaussian prior, there are similarities to the Gaussian Quadrature technique, a technique to numerically evaluate integrals [111]. In [38] Gaussian quadrature is utilized to compute the recursive Bayesian estimation integrals under a Gaussian assumption, and for certain choices of SP's in the UT, the two methods coincide.

- In [93] it is shown how a second order Taylor expansion fits the framework of a standard symmetric SP-set. When the SP's in a set approaches $\mu_{x}$ in the limit, the UT approximates the derivative. The mixed derivatives in the Taylor expansion is not completely captured by the SP's, but the UT can be interpreted as a method to approximate numerical derivatives.

In particular, the interpretation of the UT as a numerical derivative computation is later in Section 3.2 used to construct intuitive guidelines for parameter tuning. First, let us properly introduce the SP's, how they are chosen and how different parameterizations affect the SP-set.

\subsubsection{Sigma point set}

The most commonly used SP-set is symmetric around the mean $\mu_{x}$, obtained from (2.19). The distance of the SP's to the mean is scaled by $\sqrt{\frac{n_{x}}{1-\omega^{(0)}}}$, thus when $\omega^{(0)}$ approaches 1 , the SP's move further away from $\mu_{x}$.

A strategy for selecting a non-symmetric, simplex set of SP's is proposed in [43]. This SP-set requires only $n_{x}+2$ points, compared to $2 n_{x}+1$ for the standard symmetric set. Since the computational cost of the UT is proportional to the number of SP's used, the simplex set has a lower computational cost, especially when $n_{x}$ is large. Still, the strategy is rarely seen in the literature, and one possible reason could be the following: For any valid SP-set, the first two moments, mean and covariance, of the distribution are correctly represented. For the symmetric set all higher order odd moments are zero, which is also true for a Gaussian distribution. Hence, for a true Gaussian distribution an error is first introduced in the fourth order moment. The simplex SP-set gives the opportunity to introduce a skew in the set. On the other hand, if the distribution is assumed Gaussian the skew is zero. The set then has to be tuned to minimize the skew, and an error is introduced already in the third order moment. 


\subsubsection{UT parameters}

A commonly used formulation of design parameters is given in [115], where the parameters $\alpha, \beta$ and $\kappa$, introduced in [42] and [47], are used in combination to parameterize the symmetric SP-set. The SP-set is then given by

$$
\begin{array}{rlr}
\mathcal{X}^{(0)} & =\mu_{x}, & \\
\mathcal{X}^{( \pm i)} & =\mu_{x} \pm\left(\sqrt{\left(n_{x}+\lambda\right) \mathbf{P}_{x}}\right)_{i}, & \\
\omega_{m}^{(0)} & =\frac{\lambda}{n_{x}+\lambda}, & \\
\omega_{c}^{(0)} & =\frac{\lambda}{n_{x}+\lambda}+\left(1-\alpha^{2}+\beta\right), & \\
\omega_{m}^{( \pm i)} & =\omega_{c}^{( \pm i)}=\frac{1}{2\left(n_{x}+\lambda\right)}, & i=1, \ldots, n_{x},
\end{array}
$$

where

$$
\lambda=\alpha^{2}\left(n_{x}+\kappa\right)-n_{x}
$$

The posterior mean and covariance are estimated by

$$
\begin{aligned}
& \mu_{z}=\sum_{i=-n_{x}}^{n_{x}} \omega_{m}^{(i)} \mathcal{Z}^{(i)}, \\
& \mathbf{P}_{z}=\sum_{i=-n_{x}}^{n_{x}} \omega_{c}^{(i)}\left(\mathcal{Z}^{(i)}-\mu_{z}\right)\left(\mathcal{Z}^{(i)}-\mu_{z}\right)^{T} .
\end{aligned}
$$

The parameters $\alpha$ and $\kappa$ are scaling parameters that determine the spread of the SP's, and $\beta$ is introduced to minimize higher order errors in the covariance estimate. Note that the weights are allowed to be positive or negative, as long as they sum to 1 .

The original formulation of the UT [47] only includes one tuning parameter, $\kappa$, which is equivalent to setting $\alpha=1$ and $\beta=0$ in the equations above. Using $\kappa>0$, spreads the SP's further away from $\mu_{x}$, and $\kappa<0$ moves them closer, at the same time adjusting the weights so that the SP's still capture the first two moments of the distribution. With the symmetric SP-set, the third moment (skew) is always zero due to the symmetry, and a perfect representation of the fourth order moment (kurtosis) is in general not possible without extending the set with more points [46]. Scaling the SP's however, alters the kurtosis and for a Gaussian prior the kurtosis can be captured by setting $n_{x}+\kappa=3$, see [47] for details.

All SP strategies share the property that with a large $n_{x}$, the spread of the points has to be larger to capture the covariance correctly. When the SP's are spread more they capture more non-local effects, which for many nonlinearities is an unwanted effect. Hence, a way to move the points closer to the mean is needed. However, $\kappa<0$ can result in non-positive-semi-definite covariance estimates, and therefore a numerically stable scaling parameter, $\alpha$, is introduced in [42]. The parameter $\alpha \in(0,1]$ can be made arbitrarily small and $\alpha=1$ means 
no scaling. Thus, $\alpha$ cannot be used to spread the points further, only to move them closer to the mean. The incentive not to sample non-local effects is further stressed in $[111,115]$ where the small value $\alpha=10^{-3}$ in combination with $\kappa=0$ is suggested to decrease the spread of the SP's.

With the parameter $\alpha$ introduced, the SP's capture the kurtosis of a Gaussian prior if $n_{x}+\lambda=3$ with $\lambda$ defined by (3.2). For a small $\alpha$ this condition can be fulfilled by adjusting $\kappa>0$. However, as seen in (3.1) the spread of the SP's is completely determined by $\lambda$, and $\kappa>0$ cancels the scaling effect of the small $\alpha$. This redundancy between $\alpha$ and $\kappa$ should be handled as follows:

- If a decrease in the spread of the SP's is desired, use $\kappa=0$ and $\alpha<1$.

- If an increase in the spread of the SP's is desired, use $\kappa>0$ and $\alpha=1$.

Note also that for a Gaussian prior, there is a contradiction in shrinking the spread of the SP's to minimize non-local effects, and matching the kurtosis of the prior distribution. This trade off has to be considered for every specific application and the parameters need to be tuned accordingly.

By design the SP-set captures the first two moments of the prior distribution. How errors in higher order moments of the prior affect the posterior estimate of $\mu_{z}$ and $\mathbf{P}_{z}$ can be analyzed using Taylor expansions. This calculation is included in the appendix of [46] and shows that if the moments of the prior are known up to the $2 m$ th order term, the posterior mean can be correctly calculated to the $2 m$ th order term and the posterior covariance to the $m$ th order term. This is further stressed in [33], where it is shown that the posterior covariance is estimated correctly by the UT to the second order term in the Taylor expansion for the special case of a scalar $\mathbf{x}$. For a vector valued $\mathbf{x}$, the UT cannot express the mixed second order derivatives occurring in the second order term of the Taylor expansion of $\mathbf{P}_{z}$, without extending the symmetric SP-set with more points to match the prior kurtosis.

Second order errors in the Taylor expansion of the posterior covariance follow directly from the fact that the SP's fail to capture the kurtosis of the prior distribution. The parameter $\beta$ is in [42] introduced to partially minimize the higher order errors in the covariance estimate. It is argued that $\beta=2$ cancels one of the second order error terms, under the assumption of a scalar, Gaussian prior distribution. The scalar assumption should indeed be emphasized here, since it is not explicitly stated in [42] and $\beta$ is seldom changed even for non-scalar transformations $[12,35,75,119,120]$. However, even for scalar priors $\beta=2$ is not guaranteed to be optimal. Without knowledge of the specific nonlinear transformation, the canceled term is not guaranteed to be the main contributor to the error. The parameter $\beta$ should therefore be tuned not only based on the prior distribution, but also by taking the specific nonlinear function into consideration.

Table 3.1 summarizes the different parameter setups suggested by [47] and [115], and also includes the cubature transform (СТ) used in the cubature Kalman filter (CKF) [2]. Even though the CT is derived from different principles than the UT, it still fits the framework with this particular parameter setup. 
Table 3.1: Different standard parameter setups for the UT.

\begin{tabular}{lccc}
\hline Method & $\alpha$ & $\beta$ & $\kappa$ \\
\hline UT1 [47] & 1 & 0 & $3-n_{x}$ \\
UT2 [115] & $10^{-3}$ & 2 & 0 \\
CT [2] & 1 & 0 & 0 \\
\hline
\end{tabular}

Table 3.2: Approximation of mean $\mu_{z}$, and variance $P_{z}$, for three different distributions of $x$. The transformation $z=g(x)$ is given by (3.4) with the angular frequency $\omega=4 \pi$. The number of Monte Carlo runs are 100000.

\begin{tabular}{lcccccc}
\hline & $p(x)$ & \multicolumn{2}{c}{$\mathcal{N}(0,0.25)$} & \multicolumn{2}{c}{$\mathcal{N}(2.2,0.25)$} & \multicolumn{2}{c}{$\mathcal{N}(-2.2,0.0025)$} \\
Method & $\mu_{z}$ & $P_{z}$ & $\mu_{z}$ & $P_{z}$ & $\mu_{z}$ & $P_{z}$ \\
\hline UT1 & 0 & 0.028 & 1.32 & 0.26 & -1.30 & $4.27 \cdot 10^{-5}$ \\
UT2 & 0 & 1.58 & 0.18 & 2.93 & -1.30 & $4.27 \cdot 10^{-5}$ \\
CT & 0 & $2 \cdot 10^{-6}$ & 0.87 & 0.13 & -1.30 & $4.27 \cdot 10^{-5}$ \\
MCT & 0.0012 & 0.08 & 1.11 & 0.21 & -1.30 & $4.28 \cdot 10^{-5}$ \\
\hline
\end{tabular}

\subsection{Design parameter guidelines}

In this section it is demonstrated how sensitive the UT is to changes in values of the design parameters $\alpha, \beta$, and $\kappa$. Using an example of a nonlinear transformation representative for measurement functions found in real life applications, guidelines for how to choose parameter values are suggested.

\subsubsection{Measurement function example}

Consider the scalar nonlinear transformation

$$
g(x)=0.1 x^{3}+0.4 \sin (\omega x),
$$

where $\omega$ is a scalar constant. An instance of the function with $\omega=4 \pi$ is illustrated by the black curve in Figure 3.1. The function consists of a global trend, a 3rd order polynomial, with an added local sine-shaped variation. This separation of a global trend and small scale local variations can be found in measurement functions in real life applications. An example is range measurements to a rugged surface where micro variations might cause linearizations not representative for the surface, resulting in bad estimation results. This occurs in the case of LIDAR measurements to the walls in an underground mine described in detail in Section 3.4.2.

The UT is applied with the standard parameter setups for three different priors on the transformation given by (3.4). The approximated posteriors are presented in Table 3.2, where also a MCT is included for validation purposes. The different values of the design parameters give very different results when the prior uncertainty is large.

Consider the interpretation of the UT as a computation of a numerical derivative. The parameters $\alpha, \beta$, and $\kappa$ then determine how this derivative is computed. 


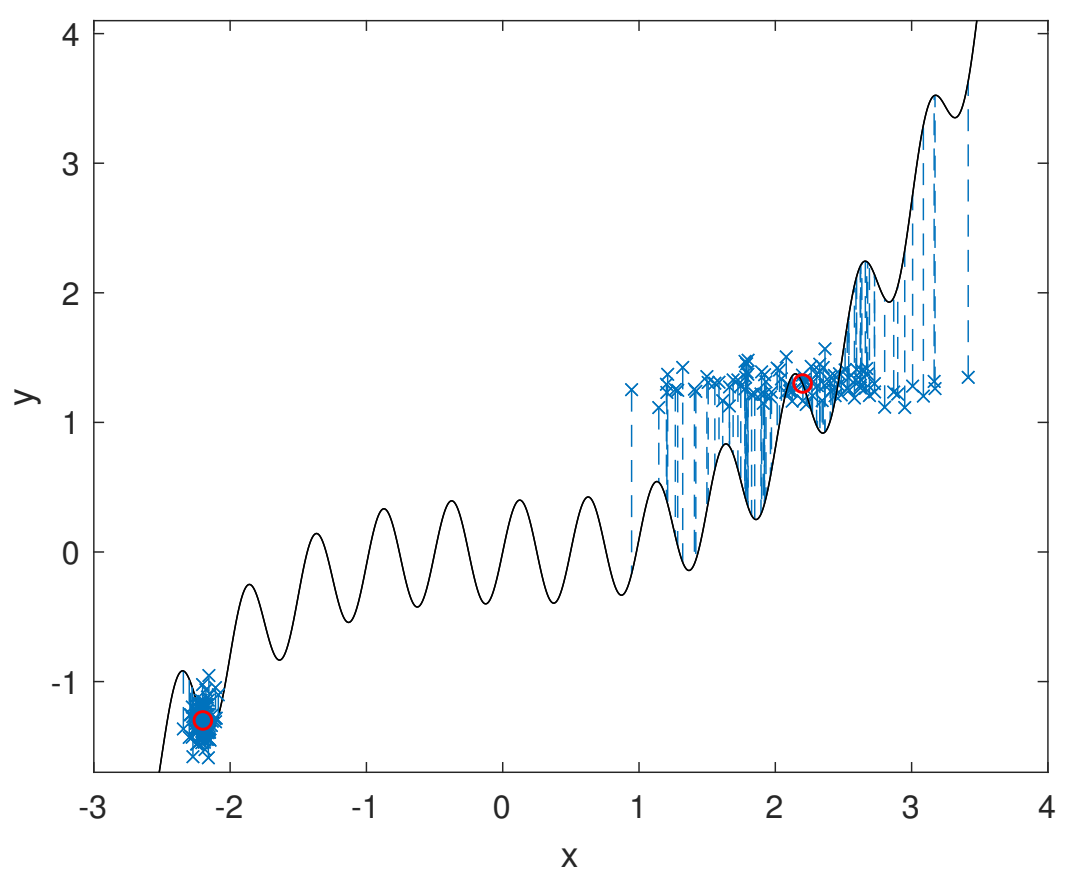

Figure 3.1: The black line shows the nonlinear transformation given by (3.4). A third order polynomial combined with a rapidly changing sinecurve, providing misleading gradient information. The angular frequency is set to $\omega=4 \pi$ and the blue crosses and lines visualizes 100 realizations of $x \sim \mathcal{N}(-2.2,0.0025)$ and $x \sim \mathcal{N}(2.2,0.25)$, respectively, with associated realizations of measurements $y$. The measurements are realized with additive white Gaussian noise of variance $\sigma_{R}^{2}=0.01$.

The transformation given by (3.4) has distinct derivatives not agreeing with the global development of the function. Such a situation could benefit from averaging the derivative by computing it based on a larger difference, capturing the area of uncertainty. This explains why the UT2 parameter setup where $\alpha$ is small (and hence also the spread of the SP's), gives poor results in Table 3.2 for the larger prior uncertainty.

\subsubsection{Guidelines}

To develop guidelines for how to choose design parameter values, this section considers the scalar function in (3.4). However, the reasoning extends to the multivariate case by allowing different parameter values in each direction.

The function in (3.4) has a global cubic behavior with added local variations with a frequency of $f=\omega / 2 \pi$. The frequency has a corresponding period, or 


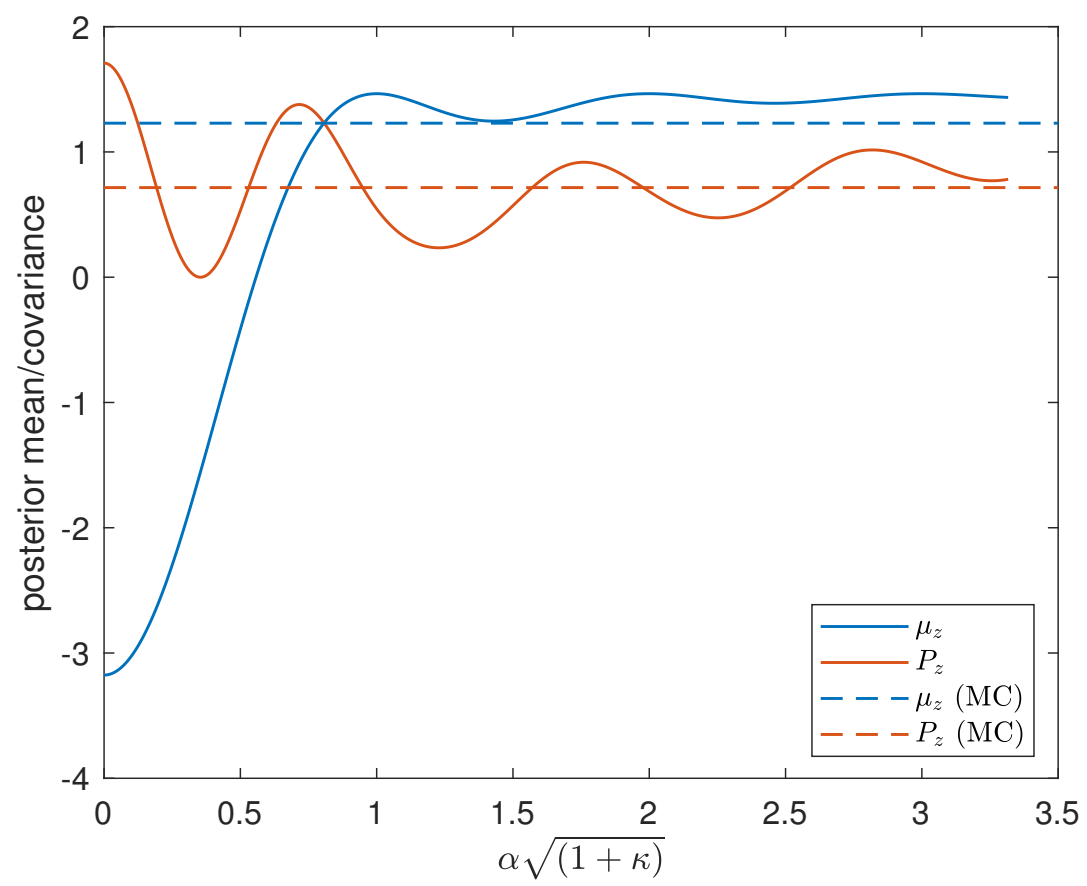

Figure 3.2: $\mu_{z}$ and $P_{z}$ for $\beta=0, \mu_{x}=2.2, P_{x}=0.25$ and $W=0.5$. The dotted horizontal lines show the result from a MCT with 100000 realizations.

wavelength in $x, W=1 / f$. By using the interpretation of the $\mathrm{UT}$ as a numerical computation of derivatives, it is reasonable to require the SP's to at least capture a wavelength of the local variations, to smooth out the local variations and instead capture the global behavior. That is, requiring $x^{( \pm 1)}-x^{(0)} \geq W$, and by inserting (3.1) this gives a condition for the tuning parameters

$$
\alpha \sqrt{1+\kappa} \geq \frac{W}{\sigma}
$$

by letting $P_{x}=\sigma^{2}$ and noting that $n_{x}=1$ for a scalar variable. Numerical examples are given in Figure 3.2, where the values on the $x$-axis are obtained by

$$
\begin{array}{ll}
\kappa=0, \quad \alpha \in(0,1], & \text { when } \alpha \sqrt{1+\kappa} \leq 1, \\
\kappa \in[0,10], \quad \alpha=1, & \text { when } \alpha \sqrt{1+\kappa}>1,
\end{array}
$$

according to the recommendations in Section 3.1.2. Note the cyclic behavior in both $\mu_{z}$ and $P_{z}$ for $\alpha \sqrt{1+\kappa} \geq \frac{W}{\sigma}$. The period of the behavior is approximately $\frac{W}{\sigma}=1$.

The parameter $\beta$ primarily affects the covariance estimates with a small spread of the SP's, see Figure 3.3. For a larger spread, above $\frac{W}{\sigma}=1, \beta$ has a low impact 


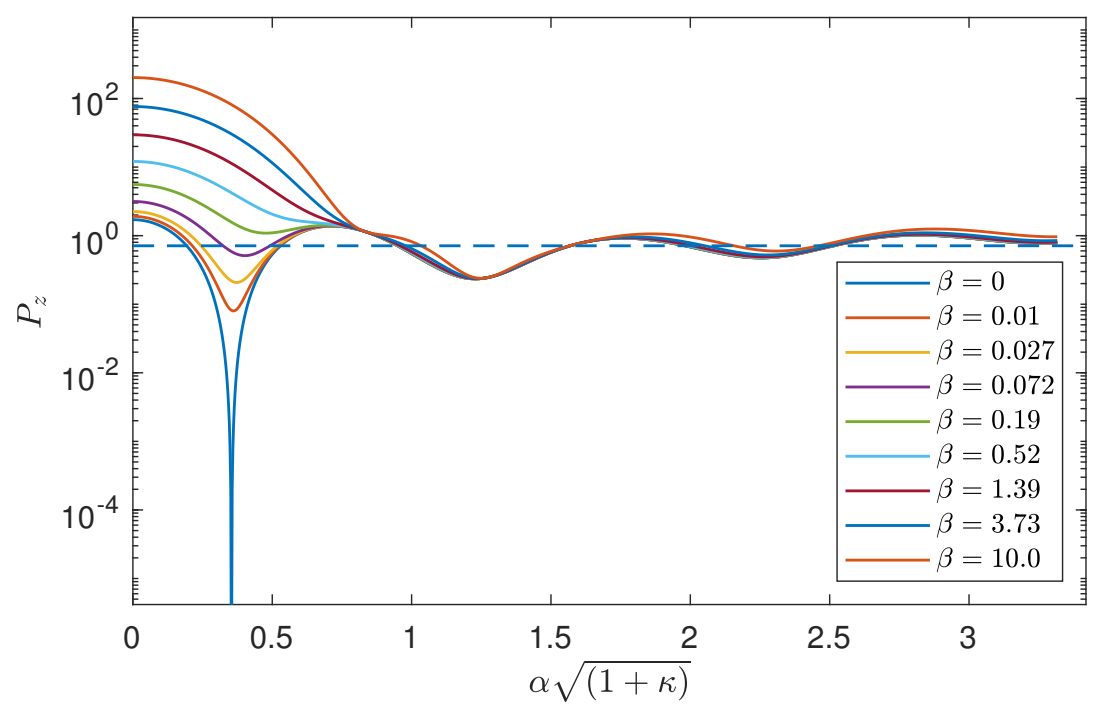

Figure 3.3: $P_{z}$ for different values of $\beta$. A priori mean and variance are given by $\mu_{x}=2.2, P_{x}=0.25$ and the wavelength is constant $W=0.5$. Small $\beta$ gives lower values of $P_{z}$ for small $\alpha \sqrt{1+\kappa}$. The dotted horizontal line shows the result from an MCT with 100000 realizations.

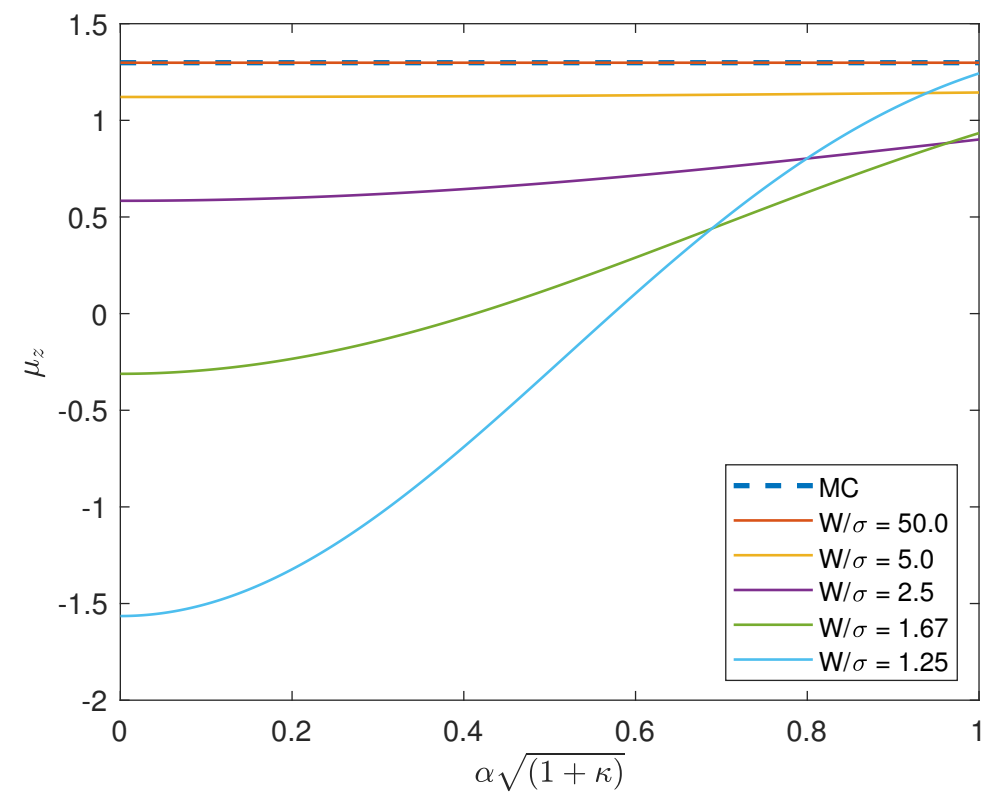

Figure 3.4: $\mu_{z}$ as a function of $\alpha(\kappa=0)$ for different values of the fraction $\frac{W}{\sigma}$. The prior mean is $\mu_{x}=2.2$, the wavelength is $W=0.5$, and the fraction is varied by using different values of $\sigma$. The dotted horizontal line shows the result from $M C T$, which in this figure coincides with the red line $(\mathrm{W} / \sigma=$ 50.0) 
on the estimate. This indicates that $\beta$ is not a crucial parameter when tuning for local variation smoothing.

If the ratio $\frac{W}{\sigma}$ is large, (3.5) would require $\alpha$ and/or $\kappa$ to be very large. UKF implementations often restrict the parameter values for computational stability, (e.g., in Mat lab $\alpha \in(0,1], \kappa \in[0,3])$. With large $\frac{W}{\sigma}$ there is no need to smooth out the local variations, and a state estimate can instead benefit from trying to capture local fluctuations to increase precision. Figure 3.4 shows that when $\frac{W}{\sigma} \gtrsim 5.0$ the transformation approximation cannot be significantly improved by spreading the SP's. For the small prior uncertainty, resulting in $W / \sigma=50$, for this problem, the approximation coincides with the MCT and is almost unaffected by scaling. The covariance approximations show similar behavior as the mean approximation in Figure 3.4.

\subsection{Auto-tuning}

The discussion in Section 3.2 indicates that choosing an optimal parameter setup from heuristics is not a straightforward task, and therefore a strategy for automatic parameter tuning is desirable. This section provides an automatic tuning procedure of the design parameters. The tuning problem is formulated as an optimization problem for a state estimation application.

The log-likelihood of an estimate described in Section 2.2.3 considers both the mean and the covariance, and the relation to the maximum likelihood makes it suitable as cost function for automatic tuning of the parameters. Assuming Gaussian distribution the log-likelihood is given by (2.32). In a simulated experiment it is possible to create data where a ground truth is available. However, in a real life situation, a true ground truth is never available. In certain scenarios measurements from high-precision external systems such as GPS or similar, may act as a ground truth. However, when no such system is available, the log-likelihood of the observations given by (2.33) is an alternative. Let the two log-likelihood functions be denoted as

$$
\begin{aligned}
& h_{1}(\mathbf{x}, \theta)=p\left(\mathbf{x} \mid \mathbf{x}^{0}\right), \\
& h_{2}(\mathbf{y}, \theta)=p(\mathbf{y} \mid \hat{\mathbf{x}}),
\end{aligned}
$$

where $\theta=\left[\begin{array}{ll}\alpha \beta & \kappa\end{array}\right]^{T}$, and $\mathbf{x}^{0}$ is the true state. The parameter tuning can then be formulated as an optimization problem

$$
\underset{\theta}{\arg \min }-h_{i}(\cdot, \theta),
$$

for any of the two cost functions.

\subsection{Simulations}

In this section simulations are performed that show how the optimization problem in (3.8) can improve the UT performance in state estimation applications 
Table 3.3: Parameters from auto-tuning.

\begin{tabular}{|c|c|c|c|}
\hline \multicolumn{4}{|c|}{$x \sim \mathcal{N}(2.2,0.25)$} \\
\hline $\mathrm{PSO}\left(h_{1}\right)$ & 1.0 & 2.61 & 2.18 \\
\hline SURR-OPT $\left(h_{1}\right)$ & 1.0 & 1.02 & 2.24 \\
\hline $\operatorname{PSO}\left(h_{2}\right)$ & 1.0 & 0.0 & 1.87 \\
\hline SURR-OPT $\left(h_{2}\right)$ & 1.0 & 0.006 & 2.09 \\
\hline
\end{tabular}

\begin{tabular}{lcrc}
\multicolumn{4}{c}{$x \sim \mathcal{N}(-2.2,0.0025)$} \\
\hline & $\alpha$ & $\beta$ & $\kappa$ \\
\hline PSO $\left(h_{1}\right)$ & 0.55 & 5.0 & 0.0 \\
SURR-OPT $\left(h_{1}\right)$ & 0.93 & 3.92 & 0.0 \\
PSO $\left(h_{2}\right)$ & 0.29 & 0.10 & 0.0 \\
SURR-OPT $\left(h_{2}\right)$ & 0.95 & 0.05 & 0.0 \\
\hline
\end{tabular}

using off-the-shelf optimization algorithms. First the simplified scalar measurement function (3.4) is considered, and then the same technique is applied to real data recorded in an underground mine environment.

\subsubsection{Simplified example}

Suppose (3.4) together with additive noise defines the measurement function for a scalar, Gaussian state variable $x$,

$$
y=0.1 x^{3}+0.4 \sin (\omega x)+v,
$$

where $v \sim \mathcal{N}\left(0, \sigma_{R}^{2}\right)$ is assumed. Figure 3.1 depicts 100 realizations of $x$ with associated measurements for two different prior means and variances. To determine the effect of the parameters, a UKF measurement update is performed to correct the state estimate. The result for the standard parameter setups given in Table 3.1 is presented in Figure 3.5, together with the result of a standard EKF. These results are inline with the observation that when $W / \sigma$ is small, the estimate benefits from spreading the SP's. Using UT1 and CT, where the spread of the SP's are greater, results in a smaller variance after the correction.

Two different off-the-shelf optimization techniques are applied to (3.8); particle swarm optimization (PSO) [53] and surrogate optimization (SURR-OPT) with radial basis functions as surrogates $[34,88]$. PSO and SURR-OPT are global optimization techniques, and for PSO the outcome is also further improved by the local optimization method pattern search $[5,55]$. The algorithms are run with standard settings as given by the Global Optimization Toolbox in Mat lab. To handle the redundancy in $\alpha$ and $\kappa$, the optimization is first performed with $\kappa=0$ allowing $\alpha$ and $\beta$ to be tuned. If the obtained $\alpha$ is close to one, the optimization is re-run with $\alpha=1$ allowing $\kappa$ and $\beta$ to be optimized. The obtained parameter values from the optimizations are presented in Table 3.3 and the results are included in Figure 3.5. In Figure 3.5(a) where $W / \sigma$ is small, there is no 


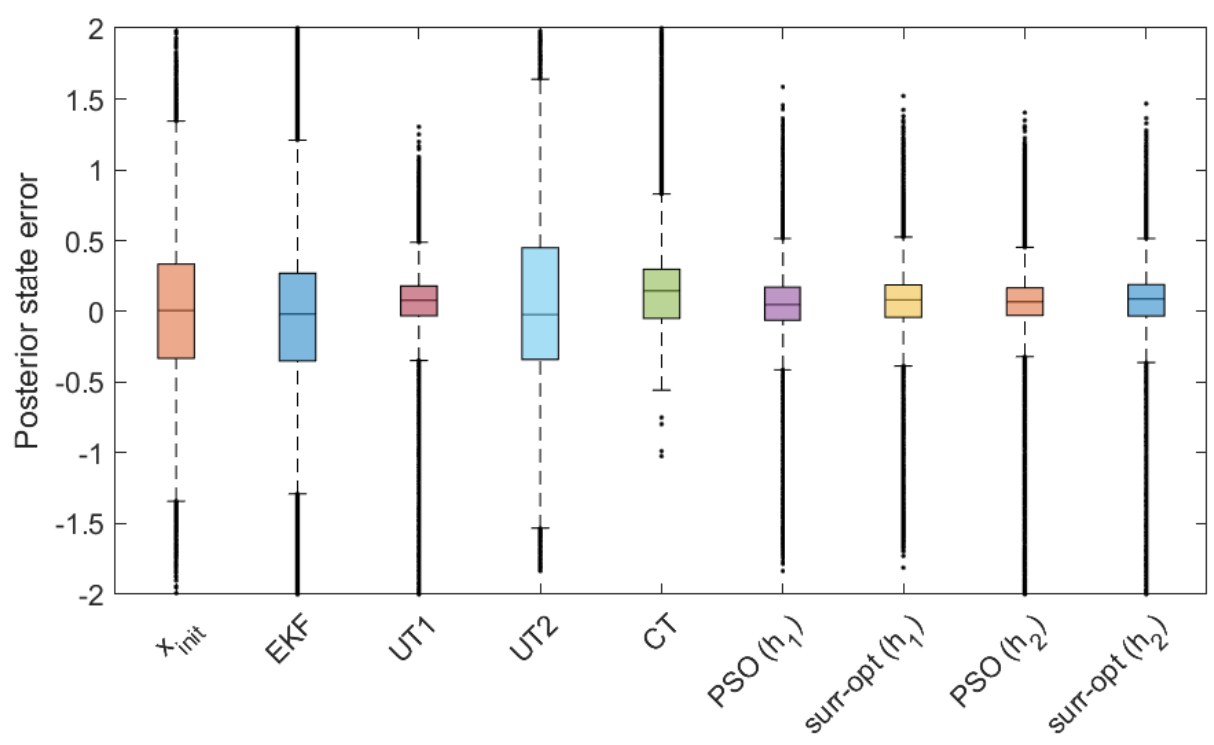

(a) $\mu_{x}=2.2$ and prior uncertainty $P_{x}=0.25$, leading to $W / \sigma=1$

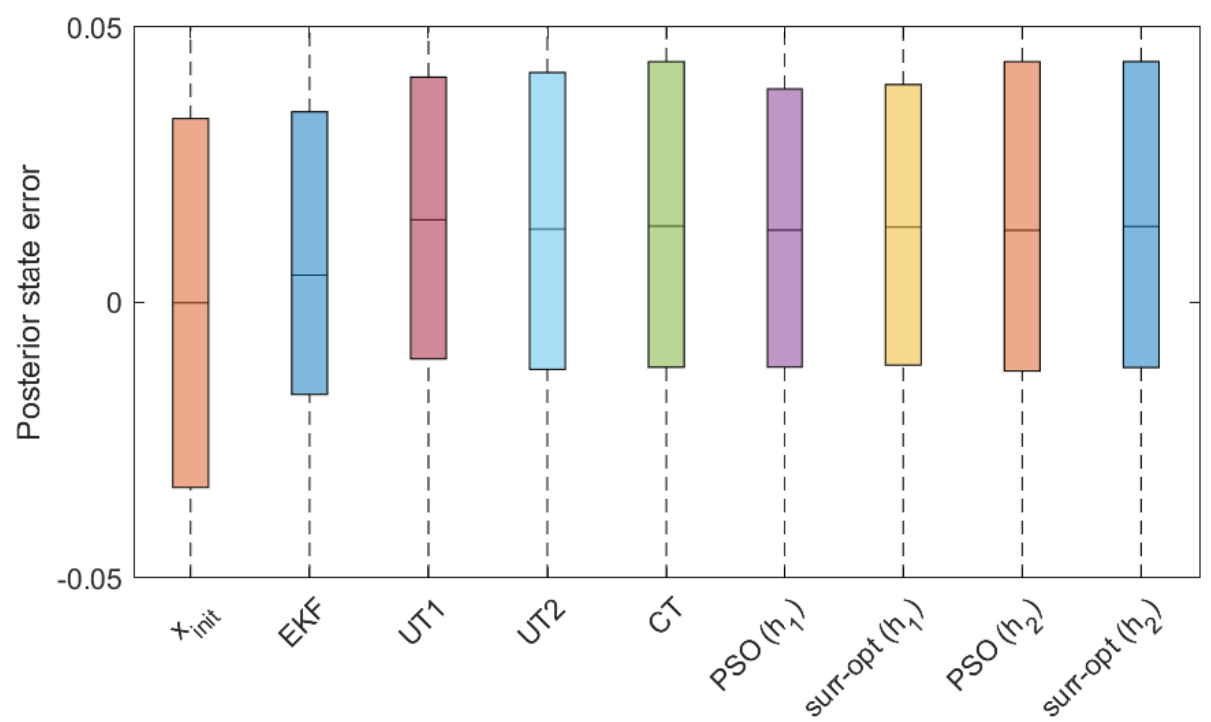

(b) $\mu_{x}=-2.2$ and prior uncertainty $P_{x}=0.0025$, leading to $W / \sigma=10$

Figure 3.5: Estimation of $x$ by performing one measurement update with EKF and UKF with different parameter values. 100000 realizations of $x$ have been used and $x_{\text {init }}$ marks the realizations of the prior distribution. The edges of the boxes indicate 25th and 75th percentiles, and the median is marked with a horizontal line. The whiskers extend to the most extreme data points not considered outliers, and outliers are marked individually with a dot. 


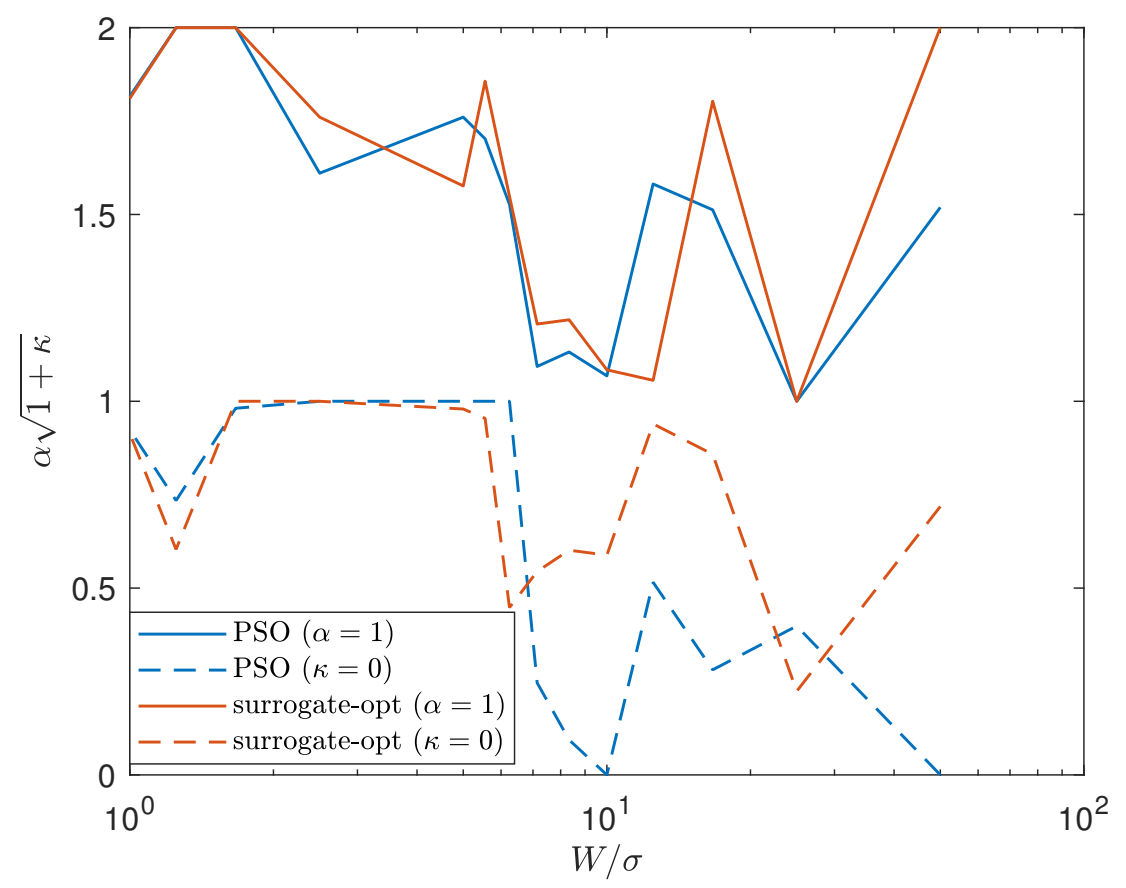

Figure 3.6: The spread in the $S P$-set obtained by the optimized parameter depends on the fraction $W / \sigma$. When $W / \sigma \lesssim 5$, parameter $\alpha$ is saturated at $\alpha=1$. The optimization is done using $h_{1}$.

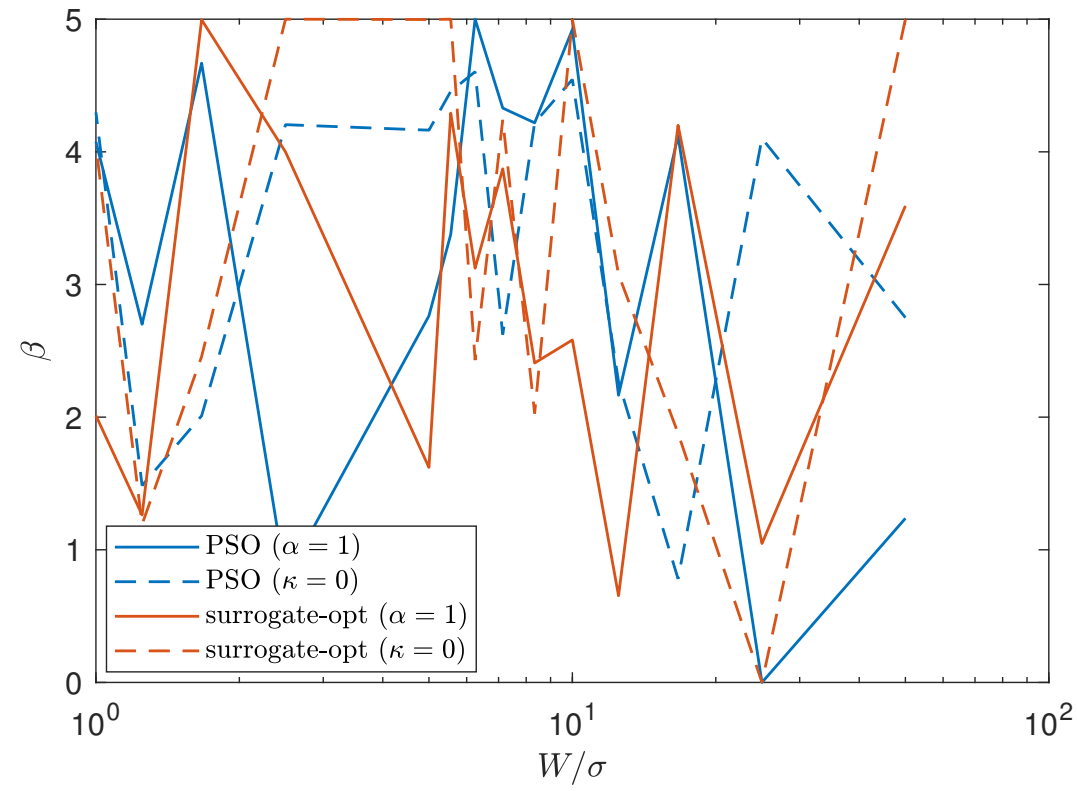

Figure 3.7: There is no clear trend in the value of the parameter $\beta$ obtained by optimization on $h_{1}$ with different fractions $W / \sigma$. 
apparent difference in the posterior error when $h_{1}$ or $h_{2}$ is used as cost function. The posterior is more accurately estimated with optimized parameters compared to the standard ones since the ability to smooth local variations is of great importance for the resulting state estimate. When $W / \sigma$ is larger, as in Figure 3.5(b), the effect of the choice of optimization cost function is more pronounced, where using $h_{1}$ gives a better result. On the other hand, the differences in the results between different parameter setups are much smaller, and the EKF performs in parity with the UKF.

The tuned parameter values in Table 3.3 are not close to any of the standard values in Table 3.1 and they also vary with the prior covariance. This shows that the local properties of the measurement equation in combination with the prior uncertainty affect the optimal parameter setup. In a dynamical system with a recursive filter update the optimal parameters therefore vary between the time updates. A natural extension to the tuning problem would be to allow the parameters to change value based on incoming observations and prior state uncertainty estimates.

Figure 3.6 shows how the spread in the optimized SP-set, changes when the ratio $W / \sigma$ is varied. For $W / \sigma \lesssim 5, \alpha$ is saturated at $\alpha=1$ which confirms the results in Figure 3.4.

The heuristic that $\beta=2$ is optimal for a Gaussian distribution is widely spread in the literature, and it is often not mentioned that this is only true if the state vector is scalar. Furthermore, it is seldom brought to attention that this is when no knowledge about the system is taken into consideration. Figure 3.7 shows the different values chosen by the optimization algorithms, and as can be seen for the particular function studied here, nothing points towards a general optimum at $\beta=2$. It is hard to see any clear trend at all in Figure 3.7 which further implies the importance of tuning the parameters for the specific problem at hand.

\subsubsection{Real data}

This section evaluates how the design parameter guidelines and auto-tuning of the parameters perform in a real life application. Data has been recorded with an underground loader (see Figure 3.8) in Epiroc's underground test mine, located outside of Örebro, Sweden. The loader is equipped with odometry, articulation angle sensor and two lidars, each having a $180^{\circ}$ field-of-view and mounted facing forward and backward, respectively. Figure 3.9 depicts a map of the test area.

The loader is localized by a UKF where odometer and articulation angle data is used as input to the time-update and LIDAR data are used as measurements. The state vector considered is $\mathbf{x}=\left[\begin{array}{lll}x & y & \phi\end{array}\right]^{\top}$ consisting of the Cartesian $(x, y)$ coordinates together with the heading $\phi$ of the vehicle. The time update is performed with the motion model,

$$
\left[\begin{array}{l}
x_{k+1} \\
y_{k+1} \\
\phi_{k+1}
\end{array}\right]=\left[\begin{array}{l}
x_{k} \\
y_{k} \\
\phi_{k}
\end{array}\right]+T\left[\begin{array}{c}
u_{k} \cos \phi_{k} \\
u_{k} \sin \phi_{k} \\
w_{k}
\end{array}\right]+\mathbf{v}_{k},
$$

where $u_{k}$ and $w_{k}$ are linear and angular velocities respectively, $T$ is the sampling 


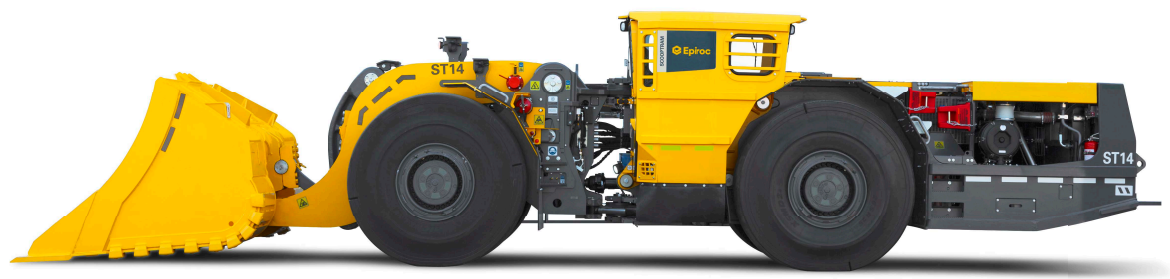

Figure 3.8: An Epiroc Scooptram14 underground loader. This is a midarticulated vehicle designed to move with 14 tonnes of material in the bucket. (Asset: Epiroc)

rate and $k$ indicates the time step. The process noise, $\mathbf{v}_{k}$, is assumed additive and $\mathbf{v}_{k} \sim \mathcal{N}(0, \mathbf{Q})$, with $\mathbf{Q}_{k}=\mathbf{Q}$ being time-invariant. The measurement equation is defined according to (2.35) with the measurement noise assumed independent and identically distributed, $\mathbf{e}_{k} \sim \mathcal{N}\left(0, \sigma_{R}^{2} \mathbf{I}\right)$.

In the recorded data $n_{y}=362$, with range measurements evenly distributed with the resolution $1^{\circ}$ and with 181 measurements for each LIDAR. While standing still in the positions marked in Figure 3.9, data is recorded to be used for tuning of the parameters. In this setting there is no ground truth data available for the positions, however, standing still enables correction of the position estimate by performing consecutive measurement updates. This state estimate can then be used to compute $\hat{\mathbf{y}}$ needed to perform auto-tuning by (3.7b).

For evaluation of the tuned parameters, data is recorded while the loader is moving. No ground truth data is available for this data either, but it is known that the vehicle drives one lap, starting in the position marked with a black circle in Figure 3.9, moving downwards in the map, and then returning to approximately the same position.

By inspecting a zoomed-in version of the map in Figure 3.10 it is seen that the walls are not smooth. Although not as regular as a sine-wave, as in the simplified example, this gives rise to local variations in the measurement function not inline with the global trend. The global trend can in this case be interpreted as the direction of a smoothed version of the wall. The 'wavelength' of the walls are by inspection of the map often in the interval $W \approx[0.5,1.5] \mathrm{m}$, although varying a lot throughout the map. According to the guideline limit $W / \sigma \simeq 5$, the standard deviation of the prior in regard to the Cartesian $(x, y)$-directions has the break point for when the UT benefits from trying to smooth the walls somewhere in the interval $\sigma \approx[0.1,0.3]$. The $\phi$-direction is somewhat more complicated since the local variation in the measurement also depends on the range of the measurement. However, with a typical range of about $10 \mathrm{~m}$ this approximately corresponds to choosing $\sigma_{\theta}$ in the order of 10-times smaller than $\sigma_{x}$ and $\sigma_{y}$. The guidelines in Section 3.2, and the simulation in Section 3.4 indicates that the optimal parameter values are highly dependent on the prior uncertainties. Therefore, 


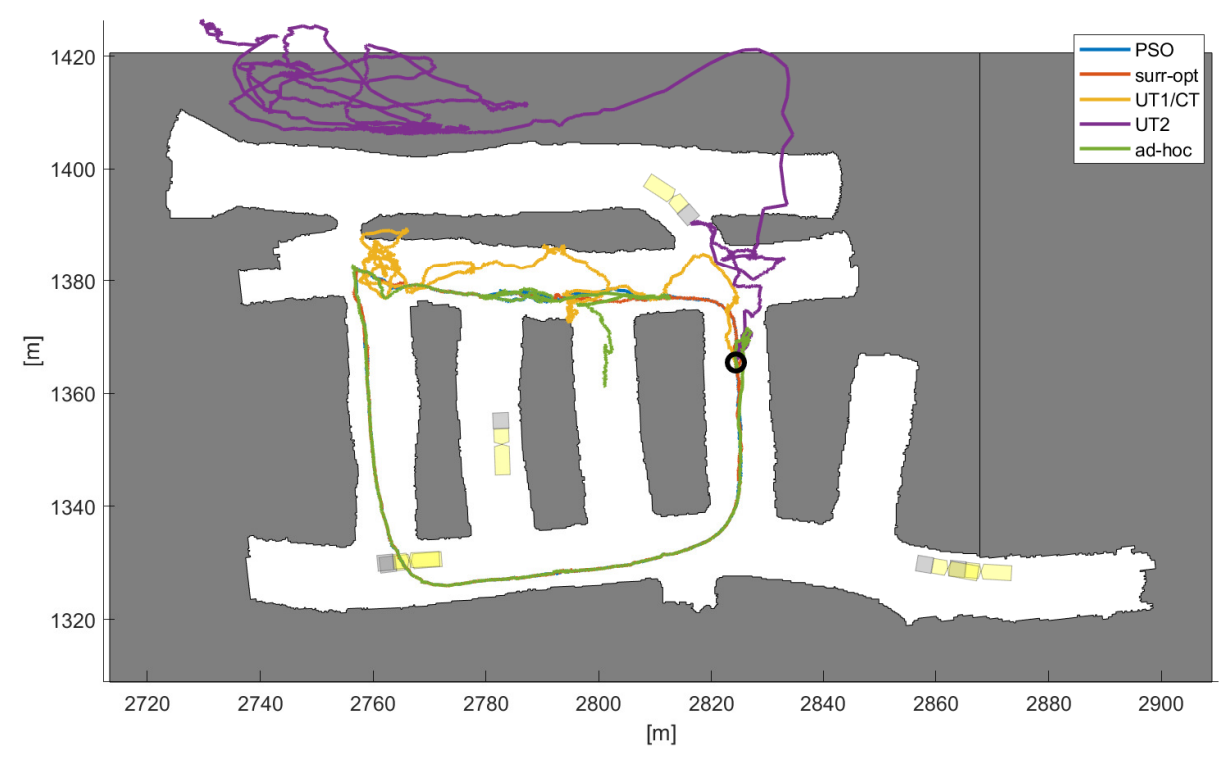

Figure 3.9: A map of Epiroc's underground test area. The yellow shadowed vehicles mark the positions where sensor data is recorded while standing still, and the black circle approximately marks the starting and stopping point when the loader was driving a full lap. The different colored trajectories are the position estimates obtained from a UKF tuned with different design parameters $\alpha, \beta$ and $\kappa$.

two different priors are considered for auto-tuning,

$$
P_{0}^{(1)}=\left[\begin{array}{ccc}
0.5^{2} & 0 & 0 \\
0 & 0.5^{2} & 0 \\
0 & 0 & 0.05^{2}
\end{array}\right],
$$

representing $W / \sigma<5$, and

$$
P_{0}^{(2)}=\left[\begin{array}{ccc}
0.1^{2} & 0 & 0 \\
0 & 0.1^{2} & 0 \\
0 & 0 & 0.01^{2}
\end{array}\right],
$$

representing $W / \sigma>5$. Choosing smaller diagonal elements than the ones in $P_{0}^{(2)}$ can give unreliable results for this application due to the nature in how the state estimates to compute $\hat{\mathbf{y}}$ are achieved. There are no guarantees that the state estimates are more accurate than $P_{0}^{(2)}$. The estimates are performed with an ad hoc tuning of the design parameters, and the map contains errors of unknown sizes and locations.

The optimization in (3.8) is performed with (3.7b) for each of the poses in Figure 3.9, with the two different prior uncertainties. The result averaged over 


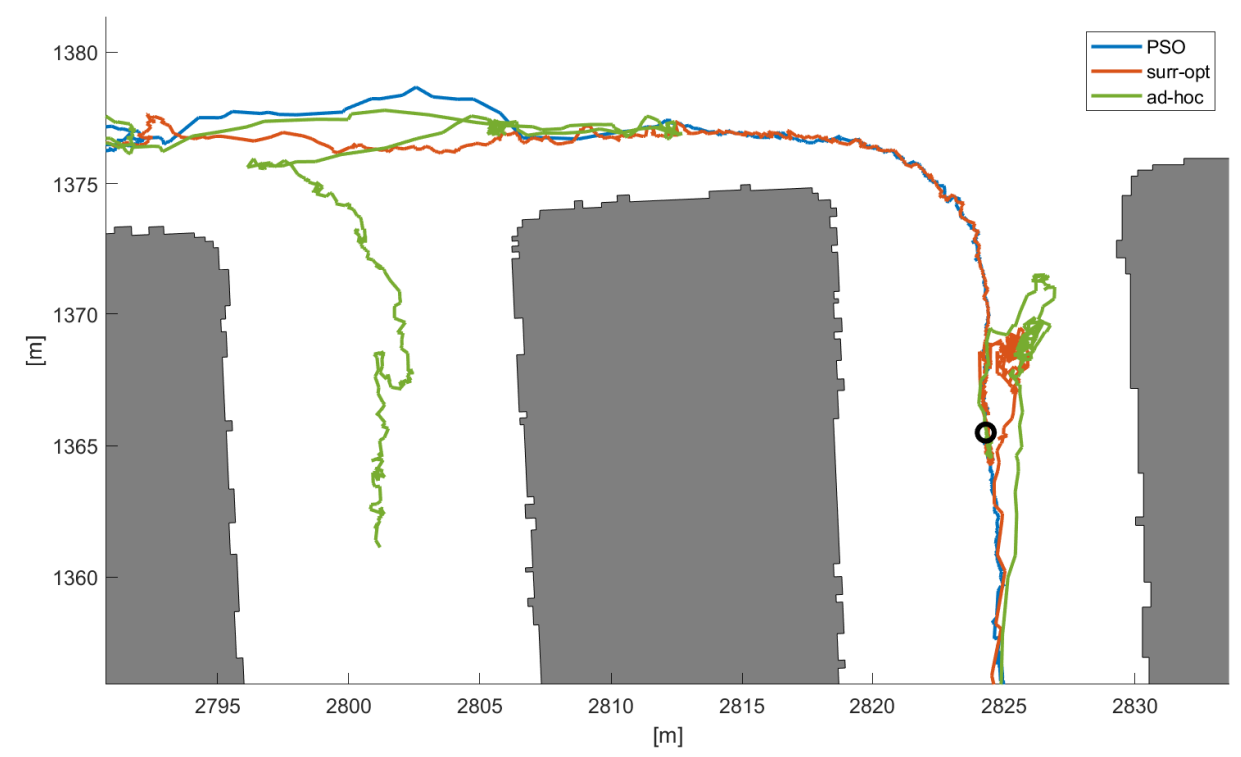

Figure 3.10: Zoomed-in version of Figure 3.9. The trajectory estimated with ad hoc parameter setup fails to perform the full lap while with auto-tuned parameters, the estimated trajectory follows the true traveling path all the way to the end.

the positions is presented in Table 3.4, with the two optimization algorithms giving similar results. By using (3.5) and $\sigma=0.5$ as in $P_{0}^{(1)}$, local variations with $W \lesssim 0.8 \mathrm{~m}$ are smoothed by the tuned UT. For the smaller prior uncertainty $P_{0}^{(2)}$ where $\sigma=0.1$, the tuned UT instead only smooths variations with $W \lesssim 0.08 \mathrm{~m}$.

The parameters obtained by the auto-tuning using $P_{0}^{(1)}$ (top two lines in Table 3.4) are used to run the UKF with the data collected while the vehicle was making one lap. The process noise covariance is set to $Q=\operatorname{diag}\left(0.002,0.002,10^{-6}\right)$, and the measurement noise is $\sigma_{R}^{2}=0.1$. In Figure 3.9, the estimated positions are shown for the different parameter setups. Using the standard values, according

Table 3.4: Parameter values obtained from real data. This is the average over all poses.

\begin{tabular}{lcccc}
\hline & $\alpha$ & $\beta$ & $\kappa$ & $\alpha \sqrt{1+\kappa}$ \\
\hline$P_{0}^{(1)}($ PSO $)$ & 1.0 & 4.2 & 1.3 & 1.52 \\
$P_{0}^{(1)}($ SURR-OPT $)$ & 1.0 & 3.1 & 1.6 & 1.61 \\
$P_{0}^{(2)}($ PSO $)$ & 0.8 & 1.6 & 0.0 & 0.8 \\
$P_{0}^{(2)}($ SURR-OPT $)$ & 0.8 & 2.0 & 0.0 & 0.8 \\
\hline
\end{tabular}


to Table 3.1, quickly results in inconsistent estimates. Note that for this application $n_{x}=3$, and therefore CT and UT1 give equal parameter values. Figure 3.10 is a zoomed version of Figure 3.9, and here it is evident that using the tuned parameter setups the UKF is able to estimate the position during the full lap. The green trajectory, depicts the estimates obtained from an ad hoc tuning with $\alpha=0.8$, $\beta=2$, and $\kappa=0$ (which by coincidence is equal to the values obtained with SURR-OPT and $P_{0}^{(2)}$ ). With these settings, the UKF almost manages to complete the lap, but at the last turn something happens and the vehicle is estimated to go down the wrong aisle.

\subsection{Summary}

In this chapter the problem of finding proper design parameter values for the UKF has been studied. The UT, internally used by the UKF, is usually controlled by three design parameters which are often set to standard values or chosen ad hoc due to lack of general guidelines. Proper choice of parameter values are essential for the performance of the UT and consequently also the UKF, and this chapter has provided useful insights into how to choose those values during filter design.

Based on a literature study of the origin of the parameters, and a simulation study, rule-of-thumbs for choosing parameter values have been derived. Furthermore, a method for auto-tuning the parameters has been formulated as an optimization problem, taking both the particular nonlinear function as well as the prior uncertainty into consideration. Formulations were provided both for the case when ground truth data is available and when it is not.

The guidelines and the auto-tuning method have been illustrated in a simulated example where the nonlinear function consists of a global trend with added local variations. It has been shown how the parameter tuning can be guided by the characteristics of the local variations. This has also been verified by an experiment on real data, collected in an underground mine environment, where the performance of the filter was substantially improved by proper tuning of the design parameters. 



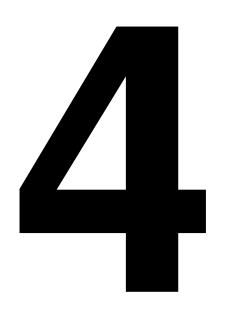

\section{Sensor selection in 2D LIDAR data}

LIDAR's typically produce a vast amount of data, resulting in possibly long processing times when used for state estimation. Methods to limit the dimensionality of the measurements, without losing quality in the resulting state estimates, can be necessary to achieve real time performance. This leads to the sensor selection problem studied in this chapter, where a thorough analysis of the information available in LIDAR measurements are used as a foundation. A map representation, and how it relates to the measurement model, is formalized in Section 4.1. In Section 4.2 the information contained in LIDAR data is analyzed, and Section 4.3 presents methods for selecting subsets of available data with retained performance of the positioning algorithm. The methods are evaluated in Section 4.4 by simulations with a mid-articulated underground loader equipped with two 2D LIDAR sensors, the same system as described in Section 3.4.2.

This chapter is a revised version of the material presented in

K. Nielsen and G. Hendeby. Sensor management in 2D lidar-based underground positioning. In IEEE $23^{\text {th }}$ Proceedings of the International Conference on Information Fusion, pages 1-6, Virtual conference, July 2020, (C) 2020 IEEE,

reused with permission of the authors and IEEE.

\subsection{Map representation}

The map of the operation area where a vehicle is to be localized can consist of line segments put together to build up the wall structure seen from above, see Figure 4.1 . The map $\mathcal{M}$ is represented by a set of corner points,

$$
\mathcal{M}=\left\{\mathbf{p}_{0}, \mathbf{p}_{1}, \ldots, \mathbf{p}_{n}\right\},
$$




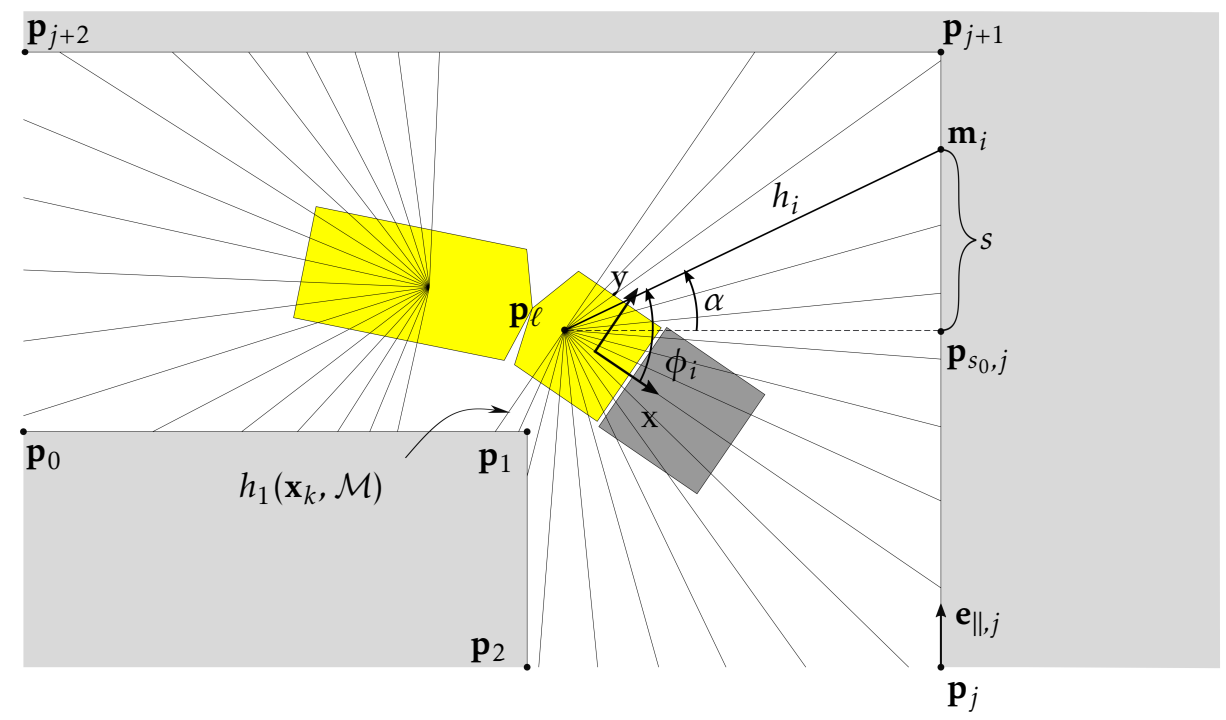

Figure 4.1: An articulated vehicle with one LIDAR mounted at the front part of the vehicle and one at the rear part. The origin of the sensor fixed coordinate system is the point in the middle of the front wheel axis. The thin lines represent a subset of available laser rays. (C) 2020 IEEE.

listed in a counter clockwise manner. The straight line segments

$$
\ell_{j}=\mathbf{p}_{j+1}-\mathbf{p}_{j}
$$

connecting the points represent the walls, $j \in[0, n-1]$. Any point on a wall segment can be parameterized as

$$
\mathbf{m}=\mathbf{p}_{s_{0}, j}+s \mathbf{e}_{\|, j},
$$

where $s$ is a scalar distance parameter, $\mathbf{p}_{s_{0}, j}$ denotes the point on $\ell_{j}$ where $s=0$ and

$$
\mathbf{e}_{\|, j}=\frac{\mathbf{p}_{j+1}-\mathbf{p}_{j}}{\left\|\mathbf{p}_{j+1}-\mathbf{p}_{j}\right\|},
$$

is the unit vector that represents the direction of the wall.

\subsubsection{LIDAR measurement model}

If the LIDAR measurement model described by (2.35) is decomposed for each individual laser ray as,

$$
\mathbf{h}\left(\mathbf{x}_{k}, \mathcal{M}\right)=\left[\begin{array}{c}
h_{1}\left(\mathbf{x}_{k}, \mathcal{M}\right) \\
h_{2}\left(\mathbf{x}_{k}, \mathcal{M}\right) \\
\vdots \\
h_{K}\left(\mathbf{x}_{k}, \mathcal{M}\right)
\end{array}\right],
$$




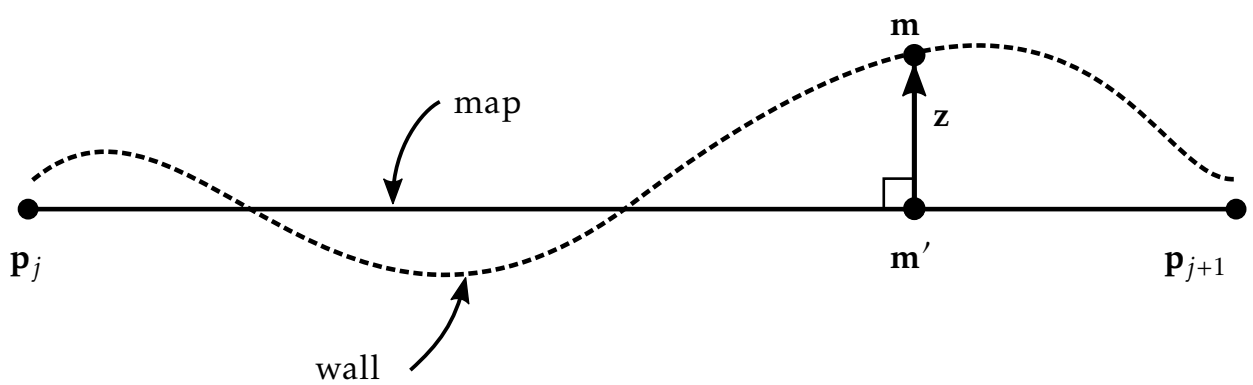

Figure 4.2: The error in a map can be described by the perpendicular disturbance $\mathbf{z}$ in a point $\mathbf{m}^{\prime}$ in the map. (C) 2020 IEEE.

the map representation presented above gives,

$$
h_{i}\left(\mathbf{x}_{k}, \mathcal{M}\right)=\left\|\mathbf{m}_{i}-\mathbf{p}_{\ell}\left(\mathbf{x}_{k}\right)\right\|+e_{k}, \quad i=1, \ldots, K,
$$

for each laser ray $i$. The point in the map where the $i$ th ray hits the wall is denoted by $\mathbf{m}_{i}$ and is in turn a function of the map $\mathcal{M}$ and the state, and $\mathbf{p}_{\ell}\left(\mathbf{x}_{k}\right)$ is the position of the LIDAR sensor immediately determined by the state, $\mathbf{x}_{k}$. The point $\mathbf{m}_{i}$ can in practice be determined using e.g., ray casting.

\subsubsection{Imperfect map representation}

In an underground mine, a map can be acquired by a pre-mapping procedure where data is collected either by the vehicle itself or by some other agent. Or, the map can be obtained from drawings made during the planning phase of the mine area. Both acquisition methods incorporate errors in the map due to the walls not being flat in the vertical direction, long straight tunnels drifting in direction or tunnels not following the planned path. The former method gives a map with a more refined resolution of irregularities of the walls and the latter gives more conceptual maps with smoother walls. wall

The error in a map can be described as a disturbance perpendicular to the

$$
\mathbf{z}=z \mathbf{e}_{\perp, j},
$$

at a point $\mathbf{m}^{\prime}$ in the imperfect map, see Figure 4.2. A point on the wall, or a point in a more accurate map, can then be written as

$$
\mathbf{m}=\mathbf{m}^{\prime}+\mathbf{z} .
$$

\subsection{Information in LIDAR measurements}

The sensor selection problem consists of finding the subset of LIDAR measurements that are as informative as possible with regard to the position estimate. The CRLB and the FIM presented in Section 2.2.1 forms a framework for this. If 
the measurements from individual rays are assumed independent, the FIM is additive

$$
\mathcal{I}(\mathbf{x})=\sum_{i=1}^{K} \mathcal{I}_{i}(\mathbf{x}),
$$

and with $e_{i} \sim \mathcal{N}\left(0, R_{i}\right), R_{i}$ independent of $\mathbf{x}$, the individual ray measurements in (4.6), each give rise to an information contribution according to (2.27)

$$
\mathcal{I}_{i}(\mathbf{x})=\nabla_{\mathbf{x}}^{\top} h_{i}\left(\mathbf{x}_{k}, \mathcal{M}\right) R_{i}^{-1} \nabla_{\mathbf{x}} h_{i}\left(\mathbf{x}_{k}, \mathcal{M}\right)
$$

\subsubsection{Information from individual rays}

Consider the derivative $\nabla_{\mathbf{x}} h_{i}$, where the arguments are dropped to simplify the notation. A large value of this derivative produces a large information contribution. The interpretation of $\nabla_{\mathbf{x}} h_{i}$, is the change in distance from the laser sensor to the wall, when the vehicle pose is perturbed. The chain rule yields

$$
\nabla_{\mathbf{x}} h_{i}=\nabla_{\mathbf{r}}\|\mathbf{r}\| \nabla_{\mathbf{x}}\left[-\left(\mathbf{p}_{\ell}(\mathbf{x})-\mathbf{m}_{i}\left(\mathbf{x}, \phi_{i}\right)\right)\right],
$$

where $\mathbf{r}=\mathbf{m}_{i}-\mathbf{p}_{\ell}$, and $\phi_{i}$ is the transmission angle of the ray, see Figure 4.1.

The first factor turns into a projection on the direction of the ray, given by the unit vector $\mathbf{e}_{\phi_{i}}$. This gives the first term

$$
-\nabla_{\mathbf{r}}\|\mathbf{r}\| \nabla_{\mathbf{x}} \mathbf{p}_{\ell}=-\mathbf{e}_{\phi_{i}}^{\top}\left(\left[\mathbf{I}^{2 \times 2} \mathbf{0}^{2 \times 1}\right]\right),
$$

which is interpreted as an information contribution in the direction of the ray that is always present independently of the map and the state.

Applying the chain rule again on the second term of (4.11) yields

$$
\nabla_{\mathbf{r}}\|\mathbf{r}\| \nabla_{\mathbf{x}} \mathbf{m}_{i}=\mathbf{e}_{\phi_{i}}^{\top} \nabla_{\mathbf{x}} \mathbf{m}_{i}=\mathbf{e}_{\phi_{i}}^{\top} \nabla_{s} \mathbf{m}_{i} \nabla_{\mathbf{x}} s=\mathbf{e}_{\phi_{i}}^{\top} \mathbf{e}_{\|, j} \nabla_{\mathbf{x}} s
$$

where the last equality is from (4.3). This term describes how the vehicle position, relative the angle of the line segment, determines in what direction the measurement is informative. All scaled by the inclination angle of the laser ray to the line segment of the wall.

In total

$$
\nabla_{\mathbf{x}} h_{i}=-\mathbf{e}_{\phi_{i}}^{\top}\left(\left[\mathbf{I}^{2 \times 2} \mathbf{0}^{2 \times 1}\right]-\mathbf{e}_{\|, j} \nabla_{\mathbf{x}} s\right),
$$

which holds if the map is perfect without errors.

\subsubsection{Imperfect map}

Now, consider the derivative of the measurement function incorporating the error representation introduced by (4.7) and (4.8). This yields,

$$
\nabla_{\mathbf{x}} h_{i}=-\mathbf{e}_{\phi_{i}}^{\top}\left(\left[\mathbf{I}^{2 \times 2} \mathbf{0}^{2 \times 1}\right]-\mathbf{e}_{\|, j} \nabla_{\mathbf{x}} s-\nabla_{s} z_{i} \mathbf{e}_{\perp, j} \nabla_{\mathbf{x}} s\right),
$$

noticing that $\mathbf{e}_{\|, j}$ and $\mathbf{e}_{\perp, j}$ now refer to a line segment in the imperfect map. The contribution from this new third term to the state estimate, explains how errors 
in the map affect the position estimate. Assuming that the imperfect map is a smoother representation of the walls not capturing all irregularities, another interpretation is the influence of roughness of the wall on the information contribution. If the non-smoothness would be captured in the map, this term gives the information contribution by the small irregularities.

The information from one single laser ray can be calculated by,

$$
\begin{aligned}
\nabla_{\mathbf{x}}^{\top} h_{i} \nabla_{\mathbf{x}} h_{i}= & {\left[\begin{array}{cc}
\mathbf{e}_{\phi_{i}} \mathbf{e}_{\phi_{i}}^{\top} & \mathbf{0}^{2 \times 1} \\
\mathbf{0}^{1 \times 2} & 0
\end{array}\right] } \\
& -\left(\mathbf{e}_{\phi_{i}}^{\top} \mathbf{e}_{\|, j}+\nabla_{s} z_{i} \mathbf{e}_{\phi_{i}}^{\top} \mathbf{e}_{\perp, j}\right)\left(\left[\begin{array}{c}
\mathbf{e}_{\phi_{i}} \\
0
\end{array}\right] \nabla_{\mathbf{x}} s+\nabla_{\mathbf{x}} s^{\top}\left[\begin{array}{ll}
\mathbf{e}_{\phi_{i}}^{\top} & 0
\end{array}\right]\right) \\
& +\left(\mathbf{e}_{\phi_{i}}^{\top} \mathbf{e}_{\|, j}+\nabla_{s} z_{i} \mathbf{e}_{\phi_{i}}^{\top} \mathbf{e}_{\perp, j}\right)^{2} \nabla_{\mathbf{x}} s^{\top} \nabla_{\mathbf{x}} s,
\end{aligned}
$$

neglecting $R_{i}^{-1}$ since it is just a scalar constant for an individual laser ray. As already mentioned $\nabla_{\mathbf{x}} s$ explains the direction of the information contribution, this is then scaled by the inclination angle of the ray to the wall and by $\nabla_{s} z_{i}$. The scalar $\nabla_{s} z_{i}$ is independent of the position of the vehicle and depends only on the map. Therefore in theory, it is possible to inspect only the map to identify regions that have a large potential of giving informative measurements. Problems arise if the resolution of the irregularities of the walls are higher than the resolution of the sensor, or the uncertainty of the positions, or if they are simply not included in the imperfect map.

The information contribution from a single laser ray is greater when the inclination angle is smaller, i.e., $\mathbf{e}_{\phi_{i}}$ is close to parallel to the wall. This is problematic since the uncertainty of the measurement increases at the same time due to the cone effect of the laser ray. It is therefore unreliable to maximize the information on this objective alone.

Using the geometry of the problem (see Figure 4.1), an explicit expression for the parameterization of a line segment can be obtained

$$
s=\frac{\left|\mathbf{p}_{s_{0}}-\mathbf{p}_{\ell}\right|}{\tan \alpha}
$$

where $\alpha=\arccos \left(\mathbf{e}_{\|, i}^{\top} \mathbf{e}_{\phi_{i}}\right)$. This yields the gradient

$$
\nabla_{\mathbf{x}} s=\frac{\left|\mathbf{p}_{s_{0}}-\mathbf{p}_{\ell}\right|}{\sin ^{2} \alpha}\left[\begin{array}{lll}
0 & 0 & 1
\end{array}\right]-\frac{\cos \alpha}{\sin ^{2} \alpha}\left[\begin{array}{ll}
\mathbf{e}_{\phi_{i}}^{\top} & 0
\end{array}\right]+\frac{1}{\tan ^{2} \alpha}\left[\begin{array}{ll}
\mathbf{e}_{\|, i}^{\top} & 0
\end{array}\right],
$$

where the first term is the contribution in the heading direction of the vehicle, and the second and third terms is in the translational directions. Also here, the problem with the contribution in each direction being greater when the inclination angle is small is present.

\subsubsection{Map corner points}

Up until now, it is assumed that which line segment a laser ray hits is known. In close vicinity to the corner points $\mathbf{p}_{j}$ this is uncertain. Here, $\nabla_{\mathbf{x}} s$ is undefined and 
adopts different values if the corner is approached along $\ell_{j}$ or $\ell_{j+1}$. The difference between the values depend on the angle between the two line segments and the inclination angle of the laser ray.

A large difference implies that the measurement is informative. Corners can be either convex or concave and a large angle between the line segments in general increases the likelihood of getting informative measurements. However, how much this effect can be utilized depends on the inclination angle of the ray.

\subsection{Sensor selection methods}

Section 4.2 provides tools to offline analyze a map to gain knowledge about which regions of the map have a potential to give particularly informative laser measurements. This section studies how the actual sensor selection can be performed with this knowledge in mind.

\subsubsection{Sensor selection by optimization}

First let us introduce a sensor selection vector

$$
\mathbf{w}=\left[w_{1}, w_{2}, \ldots, w_{M}\right]^{\top},
$$

where each $w_{m} \in\{0,1\}$ and $w_{m}=1$ indicates that the $m$ th laser measurement is selected. Using the additive property of the FIM allows for rewriting of (4.9) as

$$
\mathcal{I}_{D}(\mathbf{x})=\sum_{m=1}^{M} w_{m} \mathcal{I}_{m}(\mathbf{x})
$$

With a Bayesian approach the a priori error covariance can be taken into account [106]. The FIM is composed of two parts, $\mathcal{I}_{D}$, from the obtained data, and $\mathcal{I}_{P}$, from the a priori covariance,

$$
\mathcal{I}(\mathbf{x})=\mathcal{I}_{D}(\mathbf{x})+\mathcal{I}_{P}=\sum_{m=1}^{M} w_{m} \mathcal{I}_{m}(\mathbf{x})+\mathcal{I}_{P}
$$

By letting $\mathbf{P}$ represent the a priori error covariance, assumed Gaussian, the expression for the FIM follows,

$$
\mathcal{I}(\mathbf{x})=\sum_{m=1}^{M} w_{m} \mathcal{I}_{m}(\mathbf{x})+\mathbf{P}^{-1}
$$

Using the trace of the covariance matrix of the state estimate as a scalar performance measure, see Section 2.2.2, the sensor selection problem can be formulated as an optimization problem. Select $K$ laser measurements out of the total $M$ that 
minimizes the trace of the inverse FIM

$$
\begin{array}{ll}
\text { minimize } & \operatorname{tr}\left(\left[\sum_{m=1}^{M} w_{m} \mathcal{I}_{m}(\mathbf{x})+\mathbf{P}^{-1}\right]^{-1}\right) \\
\text { subject to } & \mathbf{1}^{\top} \mathbf{w}=K \\
& w_{m} \in\{0,1\}, \quad m=1, \ldots, M .
\end{array}
$$

Solving this optimization problem gives the best set of laser measurements in each measurement update; but it is not computationally feasible to solve, not even for rather small values of $K$ and $M$. Convex relaxations methods [21, 41] can simplify, but the problem is still too complex to be solved in real time. Greedy, but sub-optimal, approaches are therefore often used, $[40,99,116,121]$. These methods requires a proxy of the quality measure that is sub-modular to guarantee optimality [87]. The mean square error (MSE) as a performance measure is not sub-modular, but often still performs well in simulations $[99,121]$. Below, three different sensor selection strategies are presented with this in mind.

\subsubsection{Uniformly distributed (UNI)}

The least computationally demanding and most straight forward strategy, is to select rays uniformly over the transmission angle [104]. This strategy is referred to as uniformly distributed (UNI). Every $i$ th ray is selected and the result is sensitive to which ray chosen as starting index. No information about the map or the position of the vehicle is taken into consideration; but, this strategy is fast and computationally efficient. The UNI strategy is today used in applications where the computational resources cannot process all laser rays, and is therefore used as a benchmark in the experiments section.

\subsubsection{Greedy with adjacent rays (GAR)}

A computationally feasible strategy is to solve the optimization problem with a greedy approach. Solve (4.23) for one measurement, fixate it in the set of selected measurements, and then again solve (4.23) with the remaining measurements. Continue iteratively until $K$ measurements are selected. For the system of the mid-articulated loader described in Section 3.4.2, Figure 4.3 shows how the CRLB decreases when the number of laser measurements used increases. This method provides no optimality guarantees [121] but Figure 4.3 shows that the greedy approach more efficiently selects informative measurements than the UNI strategy.

Even though the greedy approach makes the problem feasible it is still too computationally heavy to perform online. If a point or region in the map is identified as informative, the selection strategy should make sure to capture that point/region even if the current position is uncertain. Therefore, it makes sense to select a burst of adjacent rays rather then selecting them one by one. Figure 4.4 indicates that the information captured in a laser scan is very concentrated to only a few laser rays. A scan with 60 uniformly distributed rays is less informative than eight wisely chosen ones. The selection strategy is then to first use the 


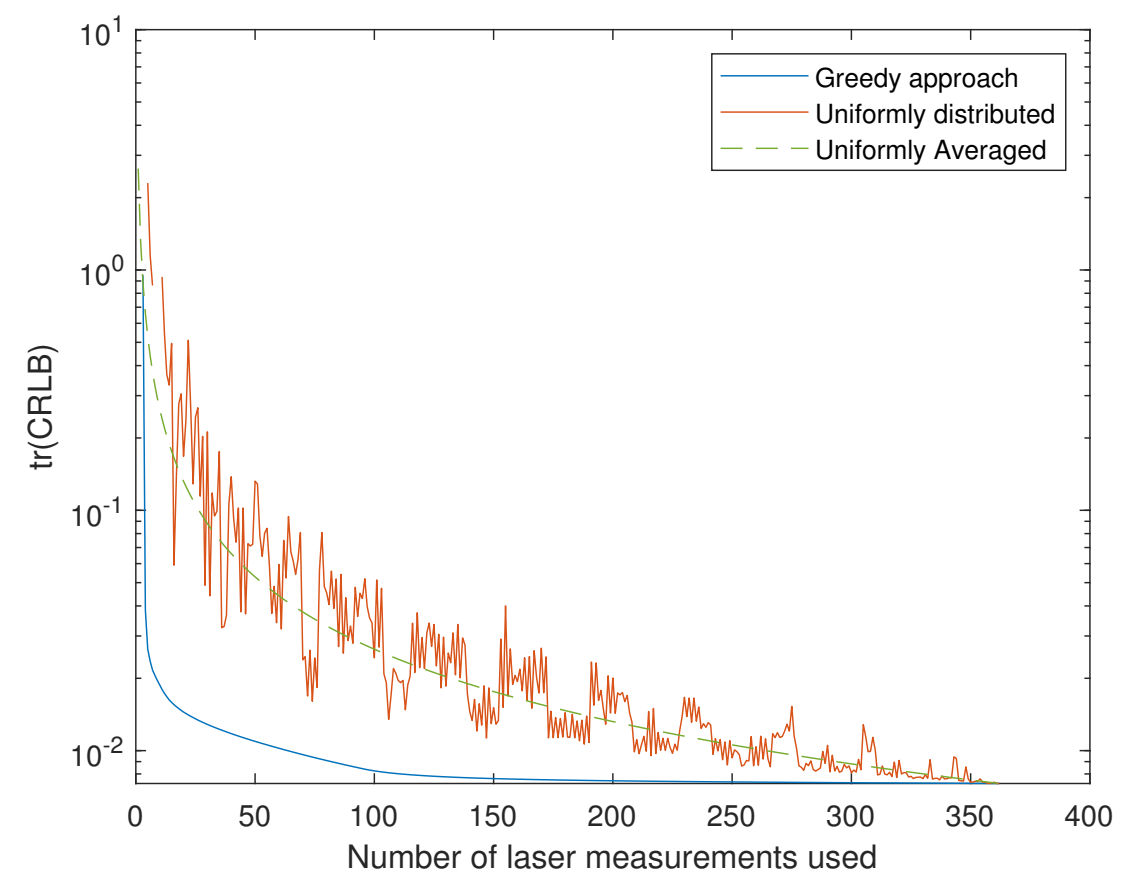

Figure 4.3: The trace of the CRLB of the pose estimate when an increasing number of laser measurements are used. This is for Pose $\boldsymbol{A}$ in Figure 4.5 in the realistic map (the blue one). The red line is for a single instance of the UNI strategy, and the dotted green line is when the UNI strategy is averaged over all possible starting index offsets of the transmission angle. (C) 2020 IEEE.

greedy approach to select a few laser rays, then add adjacent rays to compensate for the position uncertainty. Denote this strategy greedy with adjacent rays (GAR). If any of the first greedily selected rays are close to any other, there is a risk for selecting the same ray twice when adding adjacent rays. To avoid selecting a ray twice, when this situation occur, the algorithm adds more adjacent rays from the other side of the first selected one, to always obtain a fixed number of rays in the subset.

\subsubsection{Local difference in measurement data (LDM)}

To cut the computational cost even more, another strategy is to consider only the observed LIDAR measurements, and assume that the properties of the map is incorporated in that data. This selection strategy is referred to as local difference in measurement data (LDM). The idea is to compute the local difference in range between all adjacent pairs of laser rays, and then select the pairs that have a 


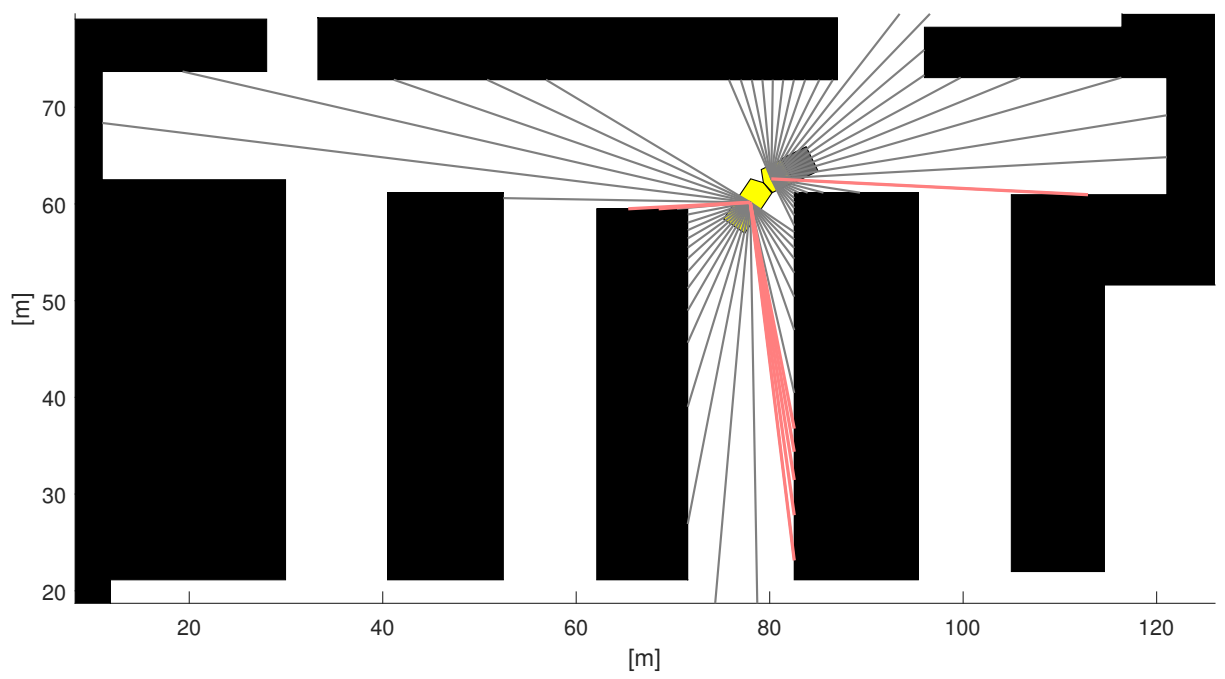

Figure 4.4: For Pose $\boldsymbol{D}$ in Figure 4.5 the trace of the CRLB is 0.0086 using 60 uniformly distributed rays. This is the black rays in the image. The 8 red laser rays are chosen according to the greedy approach, which yields only 0.0079 as the trace of the CRLB. (C) 2020 IEEE.

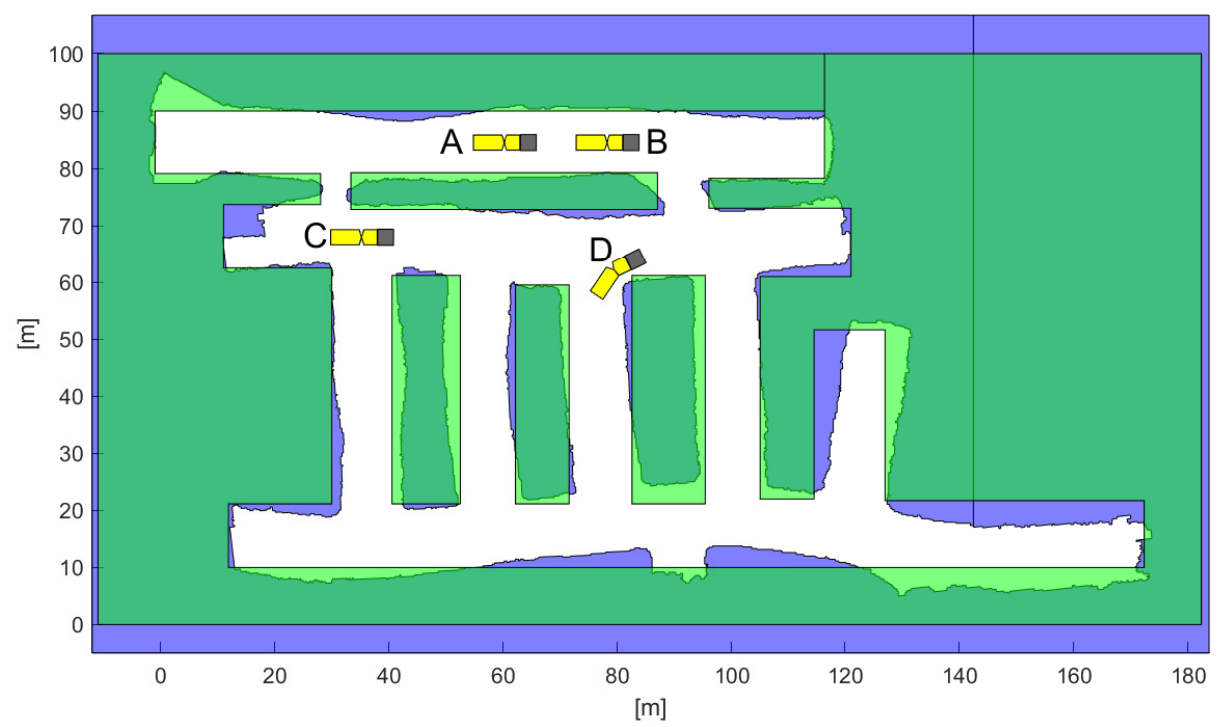

Figure 4.5: Different poses considered for one measurement update of the UKF. The two maps are overlaid with the simplified map in green and the realistic map in blue. (C) 2020 IEEE. 
high difference. This corresponds to measurements where $\nabla_{\mathbf{x}} s$, and possibly also $\nabla_{s} z$ are large in magnitude. Typically, a convex corner where the ray either hits or miss the wall are selected. There is also a possibility that the high difference corresponds to only a large $\nabla_{s} z$. This could be a large irregularity or, if the map is not perfect, it could be an error in the map. In that case the selected measurement gives false information.

Due to the position uncertainty this strategy selects a few high-difference pairs, and then add adjacent rays. This strategy however, completely ignores in which direction $(x, y$, or $\theta$ in the state vector) the sensor measurement is informative. It only finds regions that have large $\nabla_{\mathbf{x}} s$ in magnitude, but the components of $\nabla_{\mathbf{x}} s$ are completely unknown. The first term in (4.16), tells us that a measurement always gives information in the aiming direction. At least for the Cartesian $x$-, and $y$-directions. Trying to avoid a situation where all selected rays are informative in the same direction, this strategy spreads the aiming angles of the selected rays by splitting the observable half disc of each LIDAR into equally sized sections. In each section the ray pair with the largest difference is selected and adjacent rays are added. If the selected pair is close to the border of the section the adjacent rays are added from only one side.

\subsection{Experiments}

A simulation study is conducted to show how the different selection strategies UNI, GAR and LDM, performs with regard to the precision in the position estimate. The strategies are also compared to the case of using all available measurements without selection. The same system as presented in Section 3.4.2 is used here with the motion model according to (3.10).

\subsubsection{Setup}

The two horizontally mounted LIDAR sensors in total produce 362 laser measurements in each scan, 60 of them are selected for processing. With the GAR strategy, 6 rays are selected according to the greedy approach and then 9 adjacent rays are included. In the LDM strategy, each observable half disc of the laser scans is split into 3 sections, where the pair with largest numerical derivative is selected and then adjacent rays are added.

Simulations are performed with two different maps covering the same operational area. One is created from real sensor data and the other one is a synthetic version with straight walls, referred to as the realistic and simplified map, respectively. Laser scans are simulated by placing a virtual LIDAR sensor in the environment and then add white Gaussian noise with standard deviation $35 \mathrm{~mm}$ according to the specification of the physical LIDAR (sick LMS500 series).

In the operation area, four vehicle poses with different conditions for position estimation have been chosen where one measurement update of the UKF is performed, see Figure 4.5. Pose A is in the middle of a long tunnel, far away from corners that can give a high value of $\nabla_{\mathbf{x}} s$, which makes this pose hard from a 
positioning point of view. At least in the $x$-direction. In Pose B a T-junction is present just ahead of the vehicle, which puts a few points with large $\nabla_{\mathbf{x}} s$ into the field of view of the LIDAR. In Pose $C$ and $D$ the vehicle is placed in the middle of a cross-section where a lot of informative points/regions in the map is visible in the sensor measurements. This situation is easier from the perspective of position estimation, but is more complex for the selection strategies to prioritize what regions are most important.

The UKF is tuned as $\alpha=0.8, \beta=2, \kappa=0$ and the measurement noise covariance is, $\mathbf{R}=0.035^{2} \mathbf{I}$. The values of $\beta$ and $\kappa$ are commonly used standard values [115], while the value for $\alpha$ is tuned for this particular application by prior experiments.

The initial pose is perturb with a prior uncertainty with covariance

$$
\mathbf{P}_{0}=\left[\begin{array}{ccc}
10^{-4} & 0 & 0 \\
0 & 10^{-4} & 0 \\
0 & 0 & 10^{-6}
\end{array}\right]
$$

intended to reflect the desired precision in the state estimate. The precision on the heading is in accordance with the desired translational precision on the rear corner of the vehicle, the point on the vehicle furthest away from the origin of the vehicle coordinate system.

\subsubsection{Simplified map}

Figure 4.6 shows the root mean square error (RMSE) of the state vector after the correction is performed in the simplified map. For Pose A, the GAR strategy outperforms the other strategies, and in Pose $\mathrm{B}$ this is even more emphasized. In Pose C and D where a lot of corners are present LDM, and GAR gives far better results than the UNI strategy. They are even at the same order of magnitude as when all 362 rays are processed. Since the LDM strategy is computationally cheap compared to the GAR strategy, it is worth noting that they perform equally when there is a lot of information in the map. When the information is sparser in the map, LDM performs worse.

\subsubsection{Realistic map}

Figure 4.6 also shows the results from the same simulation setup applied on the realistic map. As for the simplified map, in general GAR is the best performing strategy. For Pose C and D the RMSE for all strategies are higher than for the simplified map, which means that the pose estimate cannot take advantage of the irregularities of the walls. Not even when using all 362 rays. The relatively large value of $\alpha$ causes the filter to smooth out the irregularities, according to the discussion in Chapter 3. Or, the uncertainty in the pose causes the ray to hit the wrong small feature than the expected one, resulting in false information. In the realistic map the irregularities of the walls are on the order of decimeters, and $\mathbf{P}_{0}$ is in the order of centimeters. However, (4.18) together with the results in Figure 4.7 where the correction step is performed with increasing prior uncertainty 


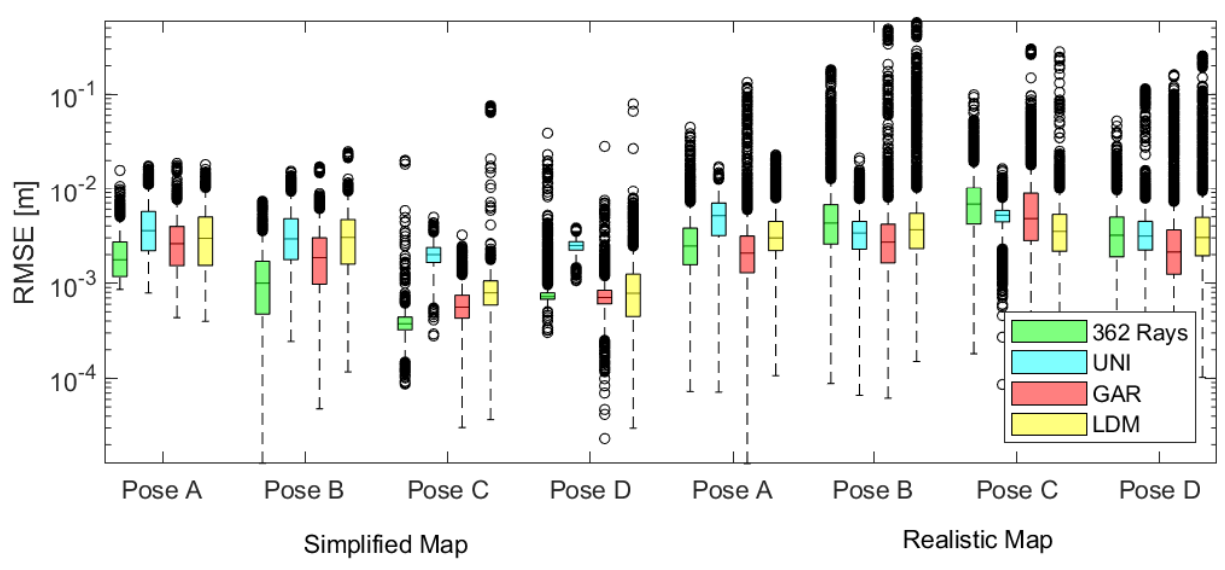

Figure 4.6: The RMSE from 10000 realizations when one measurement update is performed for different selection strategies of laser measurements. See Figure 4.5 for the vehicle poses $\boldsymbol{A}, \boldsymbol{B}, \boldsymbol{C}$ and $\boldsymbol{D}$. The edges of the boxes indicate 25 th and 75 th percentiles, and the median is marked with a horizontal line. The whiskers extend to the most extreme data points not considered outlier, and outliers are marked individually with a circle. () 2020 IEEE.

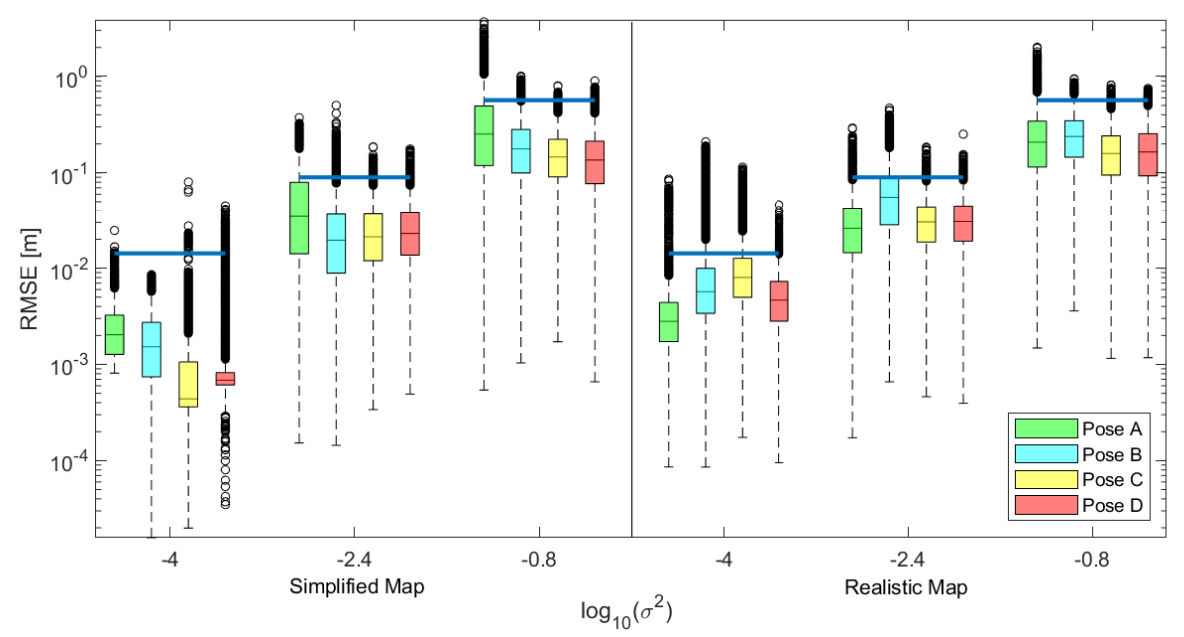

Figure 4.7: The RMSE when one correction step is performed in the different poses, when the prior uncertainty, $\mathbf{P}_{0}=\sigma^{2}\left(\begin{array}{ccc}1 & 0 & 0 \\ 0 & 1 & 0 \\ 0 & 0 & 0.1745^{2}\end{array}\right)$ is increased. The blue horizontal lines are $\sqrt{\operatorname{tr}\left(P_{0}\right)}$ for each value of $\sigma^{2}$. All 362 available laser measurements are used. The edges of the boxes indicate 25 th and 75 th percentiles, and the median is marked with a horizontal line. The whiskers extend to the most extreme data points not considered outlier, and outliers are marked individually with a circle. (O 2020 IEEE. 


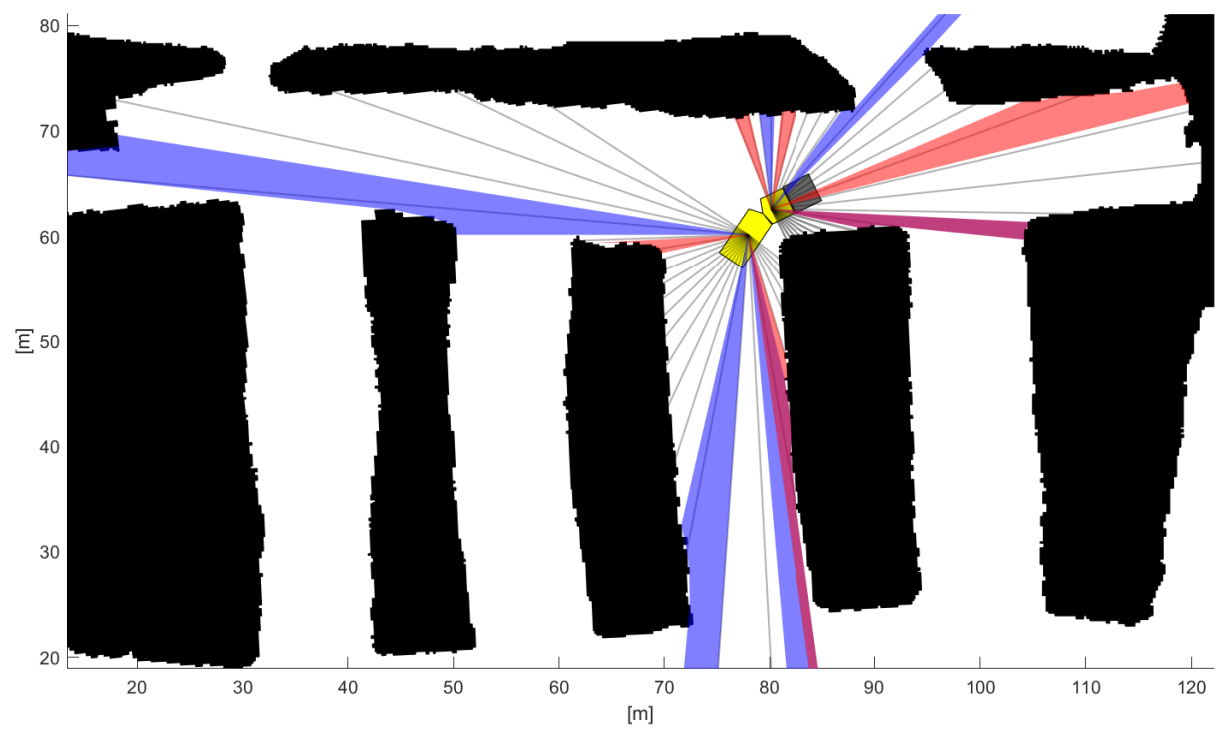

Figure 4.8: The selected rays for different strategies are visualized for Pose D. Grey lines are UNI, red is GAR and blue is LDM. The purple fields are when GAR and LDM strategies overlaps. (C) 2020 IEEE.

in the state vector, indicates that this is not enough. If it were, the RMSE would be smaller in the realistic map compared to the simplified when the prior uncertainty is decreased. The heading component in (4.18) describes how $\nabla_{\mathbf{x}} s$ gets larger in magnitude for long range measurements with small inclination angles, which means the measurements with these characteristics have a good potential of being informative. However, if $\nabla_{\mathbf{x}} s$ is larger than the irregularities of the walls, these measurements gives false information since they hit the wrong irregularity. The risk to encounter this effect increases for long range measurements with small inclination angles.

Figure 4.8 depicts how the rays are selected in Pose $\mathbf{D}$ for the different selection strategies. The GAR strategy selects fewer long range measurement than the LDM strategy and that is probably why it performs better in this situation.

There is an interesting situation in Pose B and C where using all 362 rays are worse than any of the selection strategies. Since this cannot be seen in the simplified map this is probably also an effect of the rays hitting the wrong irregularity.

\subsubsection{Map with errors}

To simulate a scenario of a map with errors, now consider the realistic map as a perfect representation of the walls, and the simplified map is the only available map. LIDAR observations are simulated from the realistic map and the position estimation is performed in the simplified map, see Figure 4.9 for results. To get the estimated pose to be more accurate after the correction step than before, $\mathbf{P}_{0}$ 


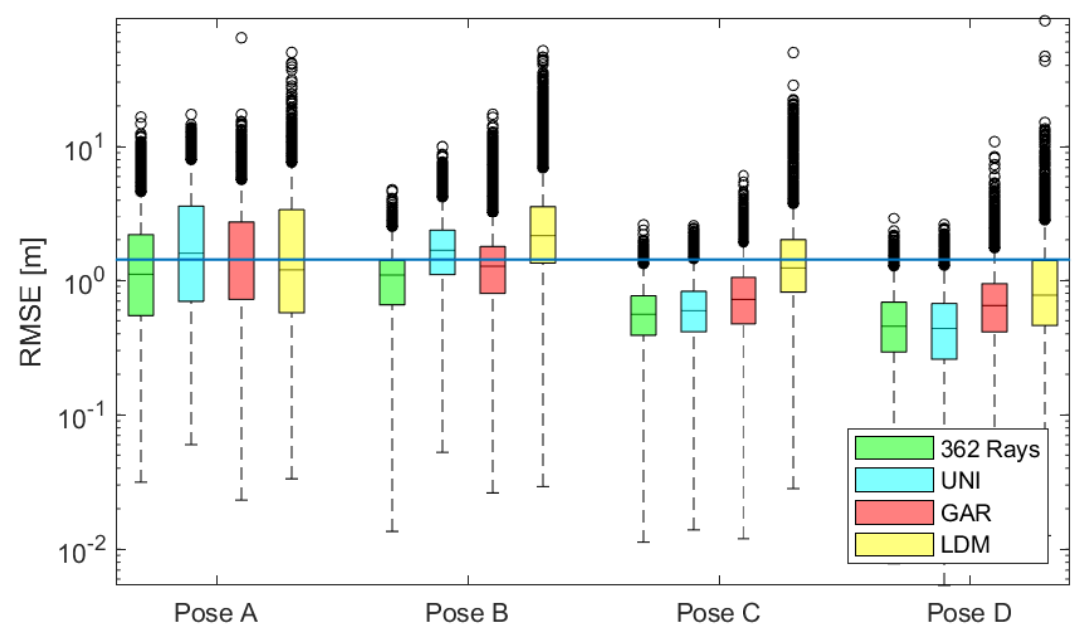

Figure 4.9: The RMSE when one correction step is performed with laser data simulated in the realistic map and the estimation done in the simplified map. The prior uncertainty, $\mathbf{P}_{0}=\left(\begin{array}{lll}1 & 0 & 0 \\ 0 & 1 & 0 \\ 0 & 0 & 0.1745^{2}\end{array}\right)$. The blue horizontal line marks $\sqrt{\operatorname{tr}\left(\mathbf{P}_{0}\right)}$. The edges of the boxes indicate 25 th and 75 th percentiles, and the median is marked with a horizontal line. The whiskers extend to the most extreme data points not considered outlier, and outliers are marked individually with a circle. (C) 2020 IEEE.

has to be increased to meter precision for this simulation. That is not surprising since the errors in the map are of this order of magnitude.

The LDM strategy performs poorly when there are errors in the map. Regions/points of interest that are captured in the observed data are simply not present in the map. That is, according to the results, worse than the opposite, as is the case for the GAR strategy. In the GAR strategy points of interest are chosen in the map and then measurements are received that are not corresponding. Worth noting is also that neither LDM nor GAR are significantly better than the UNI strategy when this type of errors are present in the map.

\subsection{Summary}

The demand of robust, highly accurate, infrastructure-free positioning systems for the underground mining industry is complicated by real-time requirements and limited computational resources. In this chapter it is shown that it is possible to reduce computational complexity while preserving quality in a 2D LIDARbased position estimate by cleverly selecting a subset of laser measurements.

By analyzing the information contribution of each laser ray measurement, ex- 
pressions have been derived that separates the contribution from the map geometry itself and the relative sensor position. The expressions expose a contradiction for long range measurements with small inclination angles. These are potentially the most informative measurements, but on the same time the measurements with largest error. Especially in maps with high resolution of irregularities of the wall, there is a risk that the laser ray hits the wrong feature in the map due to prior uncertainty of the position. Additionally, in case of an imperfect map, the features might not even be present.

An information analysis indicated what ray measurements are informative and have been used to develop two selection strategies. One is based on map geometry and the other one on observed data. Simulation experiments have shown an improvement in accuracy of the position estimate compared to the baseline strategy of uniformly selecting laser rays for both strategies. The strategy based on map geometry performed slightly better than the one base on observed data, but the latter required less computational resources. In the situation of a flawed map, simulations indicate that the developed strategies lose performance. 



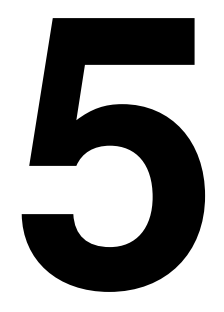

\section{Feature extraction in 2D LIDAR Data}

This chapter provides adaptations of the state-of-the-art feature extraction methods, presented in Section 2.5, to the problem of positioning a vehicle in a predefined map. Matching all laser rays from a LIDAR scan to a predefined map, is computationally expensive and extracting features is a way to reduce the dimensionality of the measurement update in a state estimation. First, in Section 5.1, detectors and descriptors are evaluated with some commonly used performance measures, which are adapted to the positioning scenario. Section 5.2 presents how the feature extraction methods are used and adapted to the underground positioning application. This is followed by experiments in Section 5.3, where the results indicate that the feature extraction methods give a state estimate robust to changes and errors in the map.

\subsection{Detector and descriptor evaluation}

The stability of a feature is defined as the ability to detect features in the same location after changing viewpoint, and regardless of sensor properties such as noise level and resolution. In the literature this is often emphasized as the most important property of a feature. In [107] a set of metrics for evaluation of feature detectors and descriptors are assessed, which in turn follows the approach developed for image processing $[73,74]$. This set of metrics is also used later in $[49,110]$. Using these criteria as a starting point, this section presents quality metrics for feature extraction methods adapted to the situation with a predefined map. The quality metrics are then used to evaluate the state-of-the-art feature extraction methods for 2D LIDAR data, FLIRT [107], FALKO [50], and BID [110], presented in Section 2.5. 


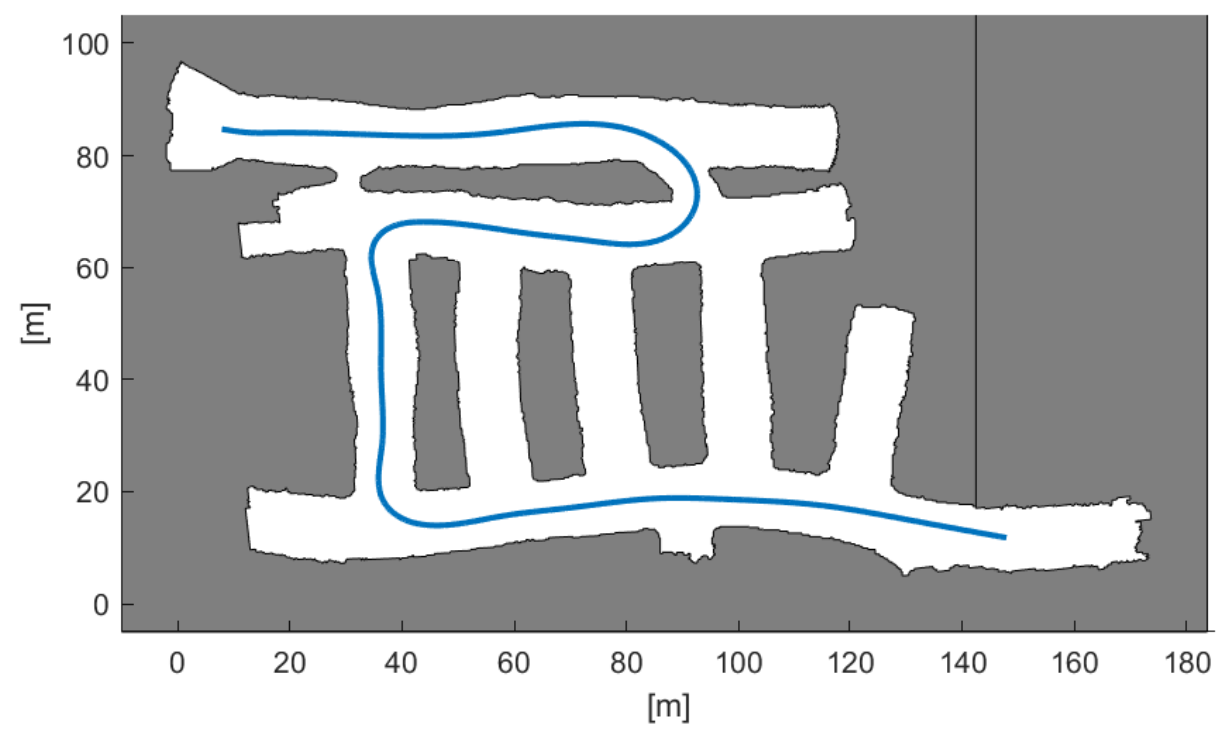

Figure 5.1: The path sampled with poses used for global stability test of feature detectors.

\subsubsection{Evaluation data}

2D laser data for feature evaluation is obtained in a simulated environment. Figure 5.1 depicts a map of Epiroc's underground test area outside of Örebro in Sweden, an area having many attributes of a typical underground mine. A path is defined so that all parts of the operation area are in the field-of-view of the LIDAR at some point along the trajectory. For each pose along the trajectory, LIDAR scans are simulated in two different ways. First, a virtual range finder is placed in the environment resulting in noise-free LIDAR scans simulating a perfect scenario with no uncertainties neither in the map nor the measurements. Secondly, measurement errors are added utilizing the SVM model presented in Section 2.3, trained on real data collected by a vehicle located in the physical area the map is covering. This results in a realistic, and stochastic, simulation of laser data.

\subsubsection{Adaptations to map positioning}

In $[50,107,110]$ the evaluation metrics are applied to datasets consisting of $2 \mathrm{D}$ LIDAR scans. For the underground application where a predefined map is available, every scan can instead be matched to keypoints extracted in the map. The procedure is therefore adapted, resulting in:

1. Find keypoints in the map. The map consists of polygons whose edges represent walls. To extract keypoints from the map it is first converted to a single point cloud by equidistant interpolation along the polygon edges. Then, a feature detector is applied to the point cloud to identify keypoints. 
2. Define a path in the map that makes as many keypoints as possible visible to the vehicle at some point along the path. It is possible to use real data collected along the path, but here simulated laser scans are obtained as described in Section 5.1.1.

3. Extract keypoints in each of the laser scans using the feature detector.

4. Associate a keypoint in a scan to a keypoint in the map if the Euclidean distance is smaller than a threshold, $d_{\max }$.

Note that the association is performed without using the descriptors, hence this procedure only measures how well different detectors can detect features in this particular environment. If the descriptors are to be evaluated the last step has to be adjusted.

\subsubsection{Detector quality measures}

This section presents quality metrics for the detectors only.

\section{Global detector stability test}

In [63] a procedure to associate extracted features from different scans is presented. A stable keypoint is detected several times as the sensor moves along the path. The covariance matrix of all keypoints in the different laser scans associated with the same feature in the map provides a measure of the stability of the feature point. The maximum eigenvalue $\lambda_{\max }$ of the covariance matrix defines a scalar metric for the stability. By taking the average over all scans in a path, this gives a measure of how sensitive a detector is to data sampling.

Another stability measure is the percentage of single points. That is the ratio of map keypoints never matched to a keypoint in any scan and gives an indication of how many unnecessary keypoints a detector finds.

How many scans along a complete path that have zero matching features is an additional metric of special importance for the localization application. In a dynamic filter approach a scan with zero matches, leads to no new information in the measurement update. Therefore, this is also included here as a supplementary stability measure.

\section{Detector repeatability}

The repeatability of a point detected from two different scans is a frequently used metric for the stability of a feature point. Consider the sets of keypoints $\mathcal{K}_{R}$ and $\mathcal{K}_{S}$ which are detected from a reference scan and from a similar scan, respectively. Assuming correct associations, the repeatability is defined as the ratio of common keypoints over the smaller of the two keypoint sets [107], i.e.,

$$
\frac{\left|\mathcal{K}_{R} \cap \mathcal{K}_{S}\right|}{\min \left\{\left|\mathcal{K}_{R}\right|,\left|\mathcal{K}_{S}\right|\right\}},
$$

where $|\mathcal{K}|$ denotes the cardinality of $\mathcal{K}$. 
Table 5.1: Parameter configurations used for the detectors.

\begin{tabular}{lccccc} 
Detector & $t_{0}$ & $t_{i}$ & $n_{k}$ & $F_{\text {min }}$ & $F_{\text {dist }}$ \\
\hline FLIRT [107] & 0.2 & 1.4 & 5 & 0.34 & 0.001 \\
& $a$ & $b$ & $\beta$ & $s_{n}$ & NMS $_{\text {min }}$ \\
\hline FALKO [50] & 0.2 & 0.07 & 4.0 & 16 & 0.2 \\
FALKO Tuned & 0.2 & 0.1 & 2.25 & 16 & 0.2 \\
& $a$ & $b$ & $T_{\text {th }}$ & $E_{\text {th }}$ & NMS $_{\text {min }}$ \\
\hline BID [110] & 0.2 & 0.07 & 3.1 & 0.03 & 0.2 \\
BID Tuned & 0.4 & 0.1 & 0 & 0.03 & 0.2 \\
\hline
\end{tabular}

When matching laser data to a predefined map repeatability is defined as the ratio between the number of associated feature points in a scan and the total number of feature points in the map visible from a particular pose of the vehicle. Again the association is purely geometric without utilizing any descriptor, to keep this as a metric for the detectors only.

\section{Detector evaluation}

The methods FALKO and BID are evaluated both using the parameter setup suggested in the corresponding papers [50,110], and with parameters manually tuned with insight from the problem, to fit this particular scenario and environment. Remember the expression defining the size of the neighborhood of a candidate keypoint from (2.47),

$$
r_{i}=a e^{b\left\|\mathbf{p}_{i}\right\|}
$$

The parameter $b$ is for FALKO and BID, manually adjusted to a larger value, which results in larger neighboring areas for longer measurements. The value in the original paper is designed to fit indoor range measurements of $\sim 10 \mathrm{~m}$, but in the underground application the ranges are typically longer. For BID also $a$ is increased to allow for larger neighborhoods also for shorter ranges. The threshold for accepting a keypoint $T_{\text {th }}$ in BID is lowered, since BID otherwise finds relatively few keypoints in this environment. On the contrary, the $\beta$ parameter for FALKO, also controlling a threshold for discarding keypoints, is adjusted to only detect the most significant keypoints.

There is always a trade-off between detecting many keypoints and only detecting the most significant ones. From the aspect of repeatability, a theoretically perfect detector would consider every point in the point cloud a keypoint, as this maximizes the repeatability score in (5.1). However, this is not desirable in practice since such keypoints are not distinctive and thus difficult to match. The detectors are therefore tuned to detect as few keypoints as possible without leaving large areas of the map without keypoints. To give a fair comparison of the three methods, equal effort has been put into the non-trivial task of tuning the 
Table 5.2: Global stability of identified keypoints with different feature detection methods.

\begin{tabular}{lccccc} 
Detector & $\begin{array}{c}\text { Keypoints } \\
\text { in Map }\end{array}$ & $\bar{\lambda}_{\text {max }}$ & $\begin{array}{c}\text { Single } \\
\text { Points }\end{array}$ & $\begin{array}{c}\text { Scans } \\
\text { without } \\
\text { match }\end{array}$ & $\begin{array}{c}\text { Repeat- } \\
\text { ability }\end{array}$ \\
\hline FLIRT & 892 & 0.0006 & $32.7 \%$ & $0.8 \%$ & 0.080 \\
FALKO Std & 1434 & 0.0019 & $48.1 \%$ & $0.0 \%$ & 0.084 \\
FALKO Tuned & 827 & 0.0016 & $55.5 \%$ & $1.0 \%$ & 0.070 \\
BID Std & 363 & 0.0020 & $60.9 \%$ & $19.1 \%$ & 0.066 \\
BID Tuned & 557 & 0.0020 & $60.0 \%$ & $11.2 \%$ & 0.067 \\
\hline
\end{tabular}

(a) Noise-free laser simulation

\begin{tabular}{lccccc} 
Detector & $\begin{array}{c}\text { Keypoints } \\
\text { in Map }\end{array}$ & $\bar{\lambda}_{\text {max }}$ & $\begin{array}{c}\text { Single } \\
\text { Points }\end{array}$ & $\begin{array}{c}\text { Scans } \\
\text { without } \\
\text { match }\end{array}$ & $\begin{array}{c}\text { Repeat- } \\
\text { ability }\end{array}$ \\
\hline FLIRT & 892 & 0.0024 & $35.9 \%$ & $8.7 \%$ & 0.042 \\
FALKO Std & 1434 & 0.0024 & $47.6 \%$ & $0.2 \%$ & 0.057 \\
FALKO Tuned & 827 & 0.0022 & $53.5 \%$ & $5.0 \%$ & 0.048 \\
BID Std & 363 & 0.0022 & $59.8 \%$ & $28.6 \%$ & 0.049 \\
BID Tuned & 557 & 0.0024 & $59.8 \%$ & $17.4 \%$ & 0.053 \\
\hline
\end{tabular}

(b) SVM laser simulation

parameter values for each of the methods. However, the sensitivity in parameter values differs for specific parameters and the different methods, resulting in various changes of parameter values. The suggested parameter setup in the corresponding papers is worst suited for the BID method, for this particular application. Therefore, this is the method where the parameter values are changed the most. See Table 5.1 for a complete list of parameter values.

All detectors have been implemented by the author in Matlab. For the FLIRT method a $\mathrm{C}++$ implementation is provided together with the paper [107]. Unfortunately this implementation cannot be used with the subsampled map as input, since it assumes laser scans containing range information. The range information is not used for the detector but only later to compute the $\beta$-grid descriptor. Therefore a Matlab implementation of a FLIRT detector taking a point cloud as input is used here, with all parameters set according to [107].

Global stability is evaluated on data produced according to Section 5.1.1 and results are presented in Table 5.2 based on $d_{\max }=0.1 \mathrm{~m}$. It is in general hard to fine-tune the methods. The tuned version of FALKO performs worse than the standard version on all measures, but still gives a slightly better position estimate as presented later in Section 5.3. The repeatability scores are in absolute values significantly lower than the results in $[50,107,110]$. That is because they are comparing scans, while the keypoints are extracted from a map in a slightly different way. The FLIRT method looks promising but is sensitive to noise. The 
repeatability score is halved when using the realistic laser simulation. On the contrary, BID is the worst performing method, but it is also the least sensitive to noise.

\subsubsection{Descriptor quality measures}

This section presents quality measures for descriptors.

\section{1-precision-recall}

To evaluate the performance of feature descriptors a 1-precision-recall curve is often used $[50,73,107,110]$. Recall is defined as the number of correctly matched keypoints with respect to the total number of true corresponding keypoints between two scans, and 1-precision is defined as the number of false matches with respect to the total number of matches found in two scans.

\section{Descriptor evaluation}

The $\beta$-grid, BSC and SDH descriptors designed for 2D laser data, are evaluated according to the 1-precision-recall criteria in $[50,110]$ with weak and comparable results. Conclusions are unison in that a pure descriptor data association is not recommended for positioning purposes, but should rather be seen as a gating rule in a geometric association method. Also, since the descriptors are designed to perform well on features found by a specific detector, it is hard to define an objective quality measure that does not also depend on the quality of the detector. Therefore, the descriptors are in this thesis only evaluated according to how they perform together with their accompanying detector, as a gating rule in a geometric association method. This is done in Section 5.3.1, once details for the position estimation procedure have been presented.

There is no straightforward way to compute the $\beta$-grid descriptors for keypoints detected in the map, since the nature of a laser ray is taken under consideration during construction. The approaching angle of a ray is used to estimate probabilities of a sector in the linear-polar histogram being occupied or not. Therefore, the descriptor gated association for FLIRT- $\beta$-grid is instead performed by matching detected keypoints to a noise free scan simulated at the true position rather than to keypoints detected from the map. This can only overestimate the performance of this descriptor causing correspondences to be better, since this decreases the demands on view-point invariance.

\subsection{Underground positioning application}

A point cloud produced by two 2D LIDAR sensors in the underground test area is provided in Figure 5.2, with a map of the operation area overlaid. In a comparison to the point clouds from the datasets in Figure 2.2, there are no sharp, well-defined corners to be found and the walls are neither smooth nor straight, yet they are continuous. This distinguishes the underground mine environment 


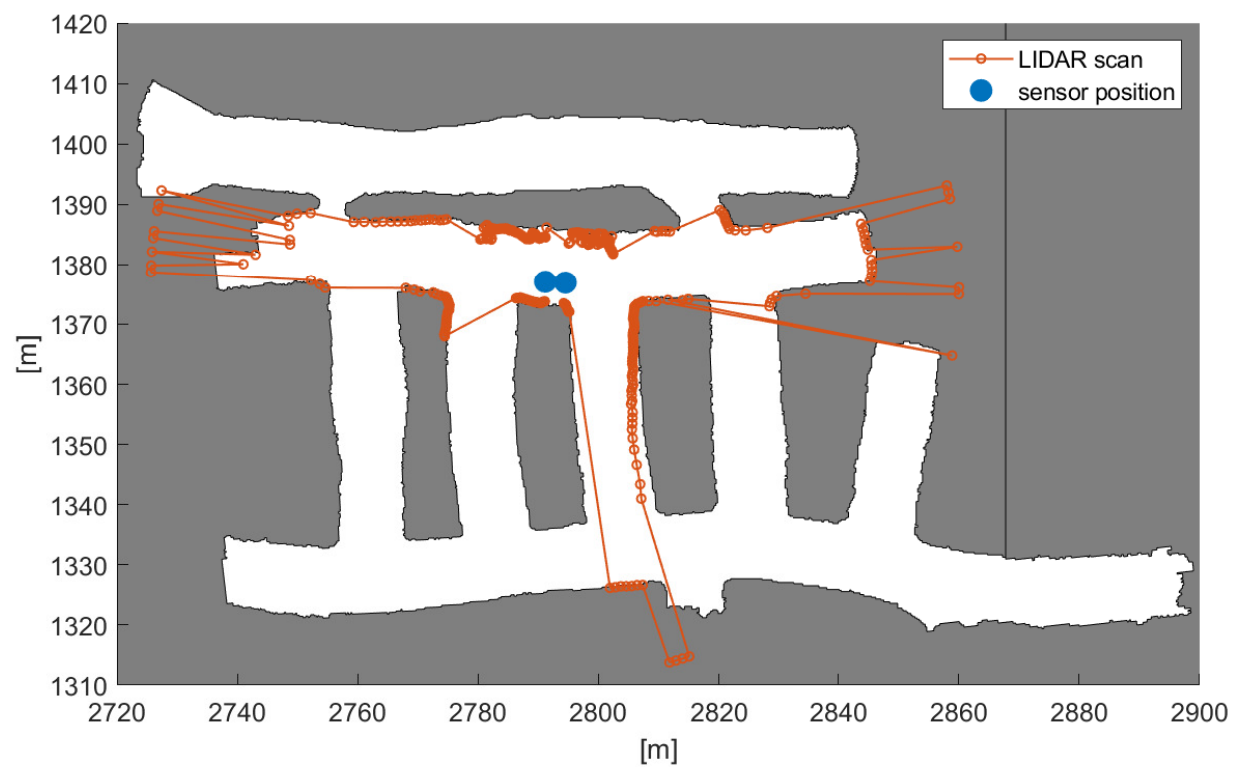

Figure 5.2: Real laser data from Epiroc's underground test area in Kvarntorp outside of Örebro in Sweden, overlaid with a map of the operation area.

from the typical indoor- as well as the typical outdoor-scenario. As seen in the figures the characteristics of measurement outliers and no-return data, follows the indoor datasets, while the scale of the operation area (a couple of $100 \mathrm{~m}$ ) have more in common with the outdoor environment. This has to be considered when applying the feature extraction methods.

The study of the underground positioning problem again utilizes the midarticulated vehicle considered in Section 3.4.2. The vehicle is equipped with odometry, IMU, and two 2D LIDAR sensors. The pose is represented by the state vector $\mathbf{x}=[x, y, \theta]^{T}$ comprising the Cartesian $(x, y)$-coordinates and the heading, $\theta$, and an UKF performs the estimation with the state transition function according to (3.10). The measurement equation for this system can either process each ray individually according to (2.35), or a feature extraction method or scan matching approach could be taken. The remainder of this section provides details on how the measurement update is implemented with the two latter strategies.

\subsubsection{Pose estimation from feature points}

When a feature extraction method is applied to the raw LIDAR data, the measurement in each update makes up a point cloud in the vehicle coordinate frame (see Figure 5.3 for definitions of coordinate systems),

$$
\mathbf{y}=\left[\begin{array}{llll}
\mathbf{p}_{1}^{T} & \mathbf{p}_{2}^{T} & \cdots & \mathbf{p}_{N}^{T}
\end{array}\right]^{T} .
$$




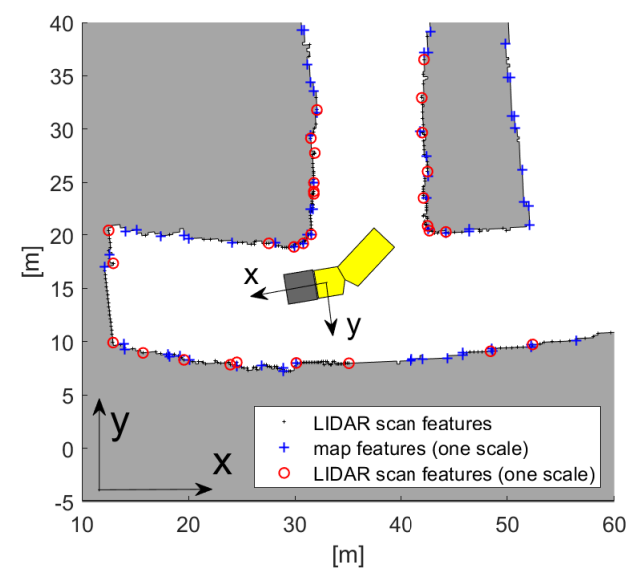

(a) Features found with FLIRT detector. Black crosses marks all features found in a LIDAR scan while the red circles highlight features in only one scale. The map features shown are associated with the same scale as the LIDAR features marked with red.

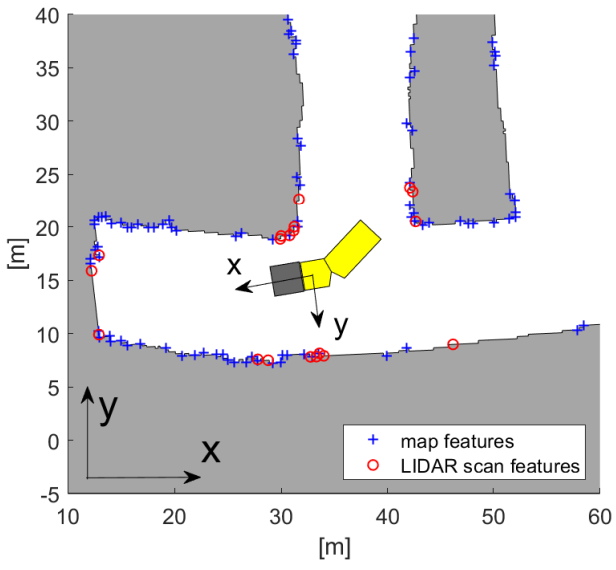

(b) Features found with tuned FALKO detector.

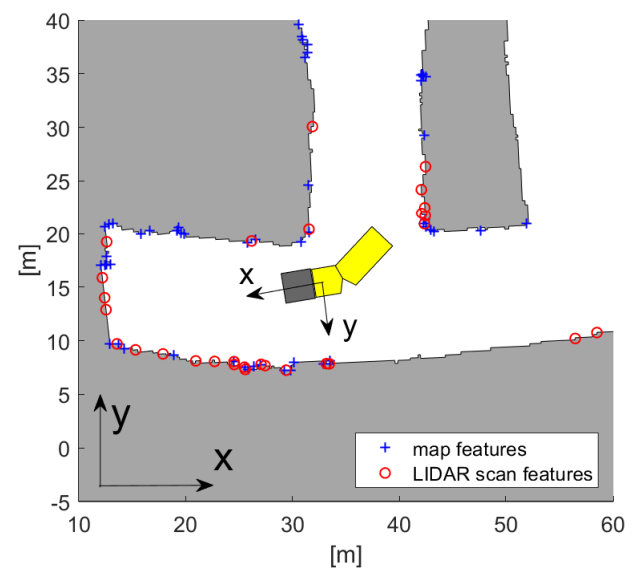

(c) Features found with tuned BID detector.

Figure 5.3: Detected features in a laser scan (red circles), are represented as a point cloud in the coordinate system with origin on the front wheel axis of the vehicle. Blue crosses marks the features found in the map and are defined in the global coordinate frame. For a perfect detector the map features and LIDAR scan features should coincide. 
Each element $\mathbf{p}_{i}, \quad i=1, \ldots, N$, is a point in the vehicle coordinate system represented by its Cartesian coordinates,

$$
\mathbf{p}_{i}=\left[\begin{array}{l}
x_{i} \\
y_{i}
\end{array}\right] .
$$

The parameter $N$ is the number of feature points where a matching feature in the map is found. The number of found features in each laser scan varies and not all detected features finds a match in the map.

To perform the UKF correction step with this setup the following preprocessing steps are performed:

1. The map is preprocessed by applying the feature detection method of choice on an interpolated version of the map, see Figure 5.3. If the method requires, descriptors for each detected feature are computed. This is done once and for all with a specific map.

2. For each new available laser scan, features are extracted according to the chosen feature extraction method. The identified features are then converted to points in the vehicle coordinate frame.

3. For each found feature a descriptor is computed according to the chosen feature extraction method.

4. The features from the laser scan are then matched to features in the map and saved as a list of currently visible map features, $\mathbf{m}$, represented as points in the global frame.

The measurement equation can then be formulated as a transform of visible feature points in the map, given in the global coordinate system, to points in vehicle coordinate frame for a given state $\mathbf{x}$,

$$
\mathbf{y}=\mathcal{R}(\mathbf{x})\left(\mathbf{m}-\mathbf{x}_{1,2}\right)+\mathbf{e} .
$$

The Cartesian components $(x, y)$ of the state vector is denoted by $\mathbf{x}_{1,2}$ and $\mathcal{R}$ is a rotation matrix. The simplistic noise model in (2.36) is used with additive measurement noise $\mathbf{e} \sim \mathcal{N}(0, \mathbf{R})$, with covariance matrix $\mathbf{R}$ assumed independent of the state.

\subsubsection{Direct scan matching}

If a data association method described in Section 2.4 is applied directly on the LIDAR data, without any preprocessing, a linear measurement equation can be used in the UKF. The UKF now acts as a low pass filter for the estimate. The outcome from a method operating directly on the raw LIDAR data is often formulated as a transform and can be converted to an indirect measurement of the state variable. The measurement equation is then reduced to the identity matrix

$$
\mathbf{y}=\mathbf{x}+\mathbf{e},
$$

with the same assumption on the measurement noise $\mathbf{e}$, as in (5.5). 


\subsection{Positioning experiments}

This section presents results from experiments conducted in the simulated mine environment described in Section 5.1.1. All laser data used in this section is simulated using the SVM model.

The UKF parameterization with $\alpha, \beta$ and $\kappa$ suggested in [115] is utilized in this evaluation, see (3.1). Parameter values are the hand-tuned values $\alpha=0.8$, $\beta=2.0, \kappa=0.0$ presented in 3.4.2. The process noise covariance matrix is set to $\mathbf{Q}=\operatorname{diag}\left(0.002,0.002,10^{-6}\right)$, and the measurement noise to $\mathbf{R}=0.5^{2} \mathbf{I}$.

\subsubsection{Measurement update}

Monte Carlo simulations of a single measurement update in the UKF algorithm is performed to analyze what accuracy the different feature extraction methods give in a position estimation application. The vehicle is positioned in the four different poses presented in Figure 4.5, with different capabilities of obtaining informative laser measurements. The map used here is the more realistic one outlined in blue in Figure 4.5. The initial pose is perturbed from the true state $\mathbf{x}_{0}=\mathbf{x}_{\text {true }}+\delta_{\mathbf{x}}$, with $\delta_{\mathbf{x}} \sim \mathcal{N}\left(0, \mathbf{P}_{0}\right)$, to simulate the position uncertainty originating from prior odometry errors.

\section{Descriptor data association}

As mentioned in Section 5.1.4, an evaluation of the descriptors is now conducted by comparing purely geometric data associations to geometric associations using the descriptors for gating. The descriptors $\beta$-grid, BSC, and SDH are used in conjunction with the detectors FLIRT, FALKO and BID, respectively, and 10000 realizations of a complete UKF measurement update are performed with an initial perturbation of the position obtained by sampling from $\delta_{\mathbf{x}} \sim \mathcal{N}\left(\mathbf{x}^{0}, \mathbf{P}_{0}\right)$ with,

$$
\mathbf{P}_{0}=\left[\begin{array}{ccc}
1 & 0 & 0 \\
0 & 1 & 0 \\
0 & 0 & 0.0076
\end{array}\right]
$$

Each realization also sample its own laser scan measurement noise from the SVM model. All descriptor histograms are constructed with $r_{n}=4$ and $\alpha_{n}=12$ as suggested in the original papers $[50,107,110]$, and features in the map within a radial distance $\left(d_{\max }\right)$ of three standard deviations of the a priori uncertainty are considered matching candidates. For keypoints with a difference in orientation smaller than a threshold $d_{\alpha}$, the keypoint with the minimum distance according to the distance function associated with the used descriptor, is considered a match. Matched features are then used as measurements in the UKF update. For FALKO-BSC, $d_{\alpha}=\pi / 6$ and for FLIRT- $\beta$-grid and BID-SDH, $d_{\alpha}=\pi / 10$ is used. The values are chosen manually by performing simulations and with knowledge of the methods and the characteristics of the specific problem. A larger threshold is used for FALKO-BSC since the orientations of these features are more uncertain, due to how they are computed. 


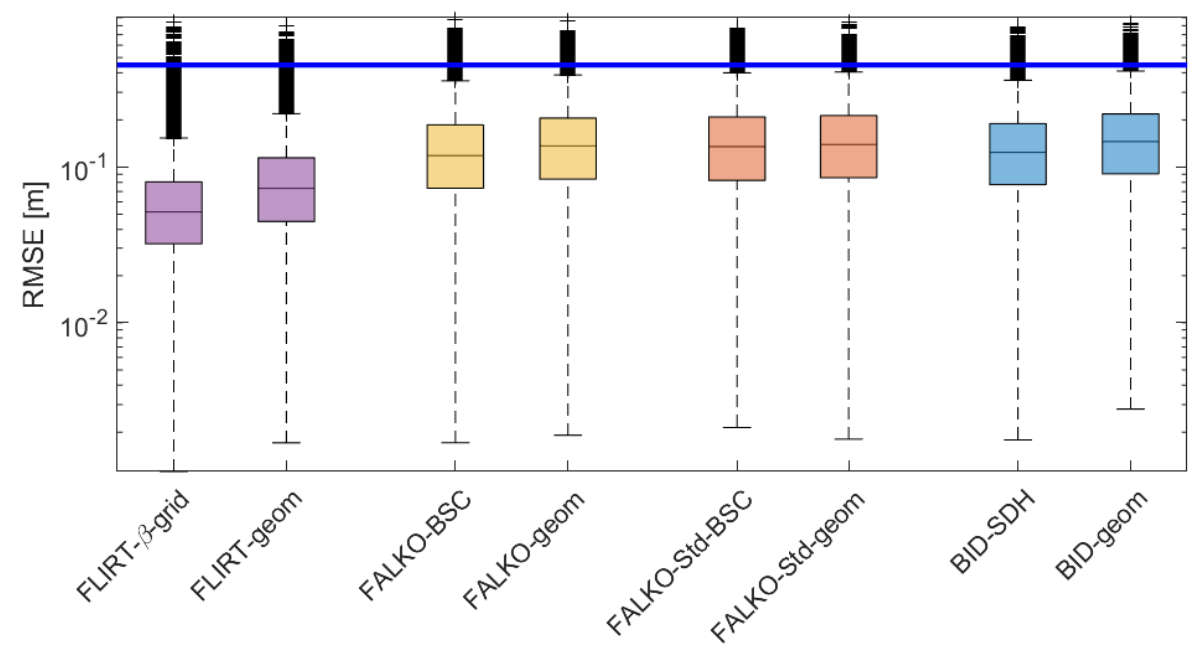

Figure 5.4: RMSE after one measurement update based on 10000 realization in each of the four poses in Figure 4.5. Different feature detectors paired with a descriptor-gated or pure geometric data association are used. The blue horizontal line represents the a priori error, $\sqrt{\operatorname{tr}\left(\mathbf{P}_{0}\right)}$. The edges of the boxes indicate 25 th and 75 th percentiles, and the median is marked with a horizontal line. The whiskers extend to the most extreme data points not considered outlier, and outliers are marked individually with a cross.

In Figure 5.4 the descriptor gated data associations is compared to pure geometric nearest neighbor data associations with $d_{\max }=0.1 \mathrm{~m}$ as threshold. The result confirms earlier results that the descriptors do not add much value when a fairly good initial value can be fed to a pure geometric association. FLIRT is the only method where the usage of the descriptor actually makes a significant difference. However, the descriptor experiment is in this case expected to overestimate the performance due to the customization that had to be made to the evaluation of the $\beta$-grid descriptor described earlier in Section 5.1.4. The tuned parameter setup of FALKO is in this experiment, unlike the results in Table 5.2, resulting in marginally lower errors than the default parameter setup. For BID, the tuned parameter setup in Table 5.1 is used. With standard parameter values, BID gives so few keypoints in the map that for many of the poses there exists no keypoints in the field-of-view.

\section{Prior uncertainty}

To compare the feature extraction methods to a standard ICP method and the different sensor selection methods described in Chapter 4, the measurement update is performed for a number of different values of $\mathbf{P}_{0}$. RMSE of 10000 realizations is depicted in Figure 5.5, again using the SVM to simulate laser data. (Note that 


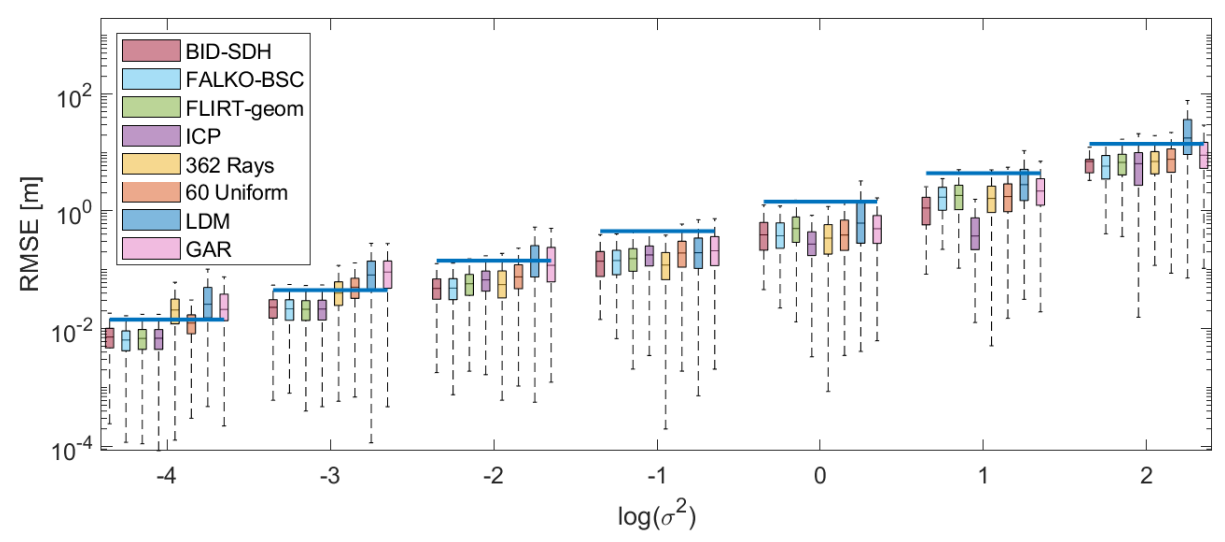

Figure 5.5: RMSE for 10000 realizations of a measurement update step with different methods and different variance of initial perturbation. The horizontal blue line is the square-root of the trace of the initial covariance matrix $\mathbf{P}_{0}=\sigma^{2}$ diag $(1,1,0.0076)$. ' 60 Uniform', LDM and GAR are the different strategies for selecting subsets of the measurement data presented in Chapter 4 . The edges of the boxes indicate 25 th and 75 th percentiles, and the median is marked with a horizontal line. The whiskers extend to the most extreme data points not considered outlier, and outliers are marked individually with a cross.

this is not 10000 replicated laser scans, but 10000 realizations of the distribution given by the SVM model.) Data association for FALKO and BID are performed with the descriptor gated geometric data association, and for FLIRT a pure geometric association is used. For ICP, a basic variant using the point-to-plane error metric without outlier rejection is used [26]. The method, denoted '362 Rays' in Figure 5.5, utilizes all 362 available laser readings and the measurement equation where the rays are processed individually, according to (2.35). The feature extraction methods perform in this evaluation in parity with the '362 Rays'-method. For FALKO, the tuned parameter setup is used in this case. It is worth noting that FALKO requires significantly less computational resources than all of the other methods. The method '362 Rays' gives the best estimate when $\sigma^{2}=0.1$ in $\mathbf{P}_{0}=\sigma^{2} \operatorname{diag}(1,1,0.076)$, which is not surprising since this is the setup the UKF parameters $\alpha, \beta$, and $\kappa$, are tuned for.

\subsubsection{Trajectory}

A trajectory is prepared with accompanying laser data, simulated using the SVM model, to investigate how the feature extraction methods perform in realistic dynamic position estimation. The trajectory is given in Figure 5.6 and the vehicle starts from standing still at the leftmost point. This trajectory is representative for movements of the vehicle in this environment, exposing the system to, e.g., 


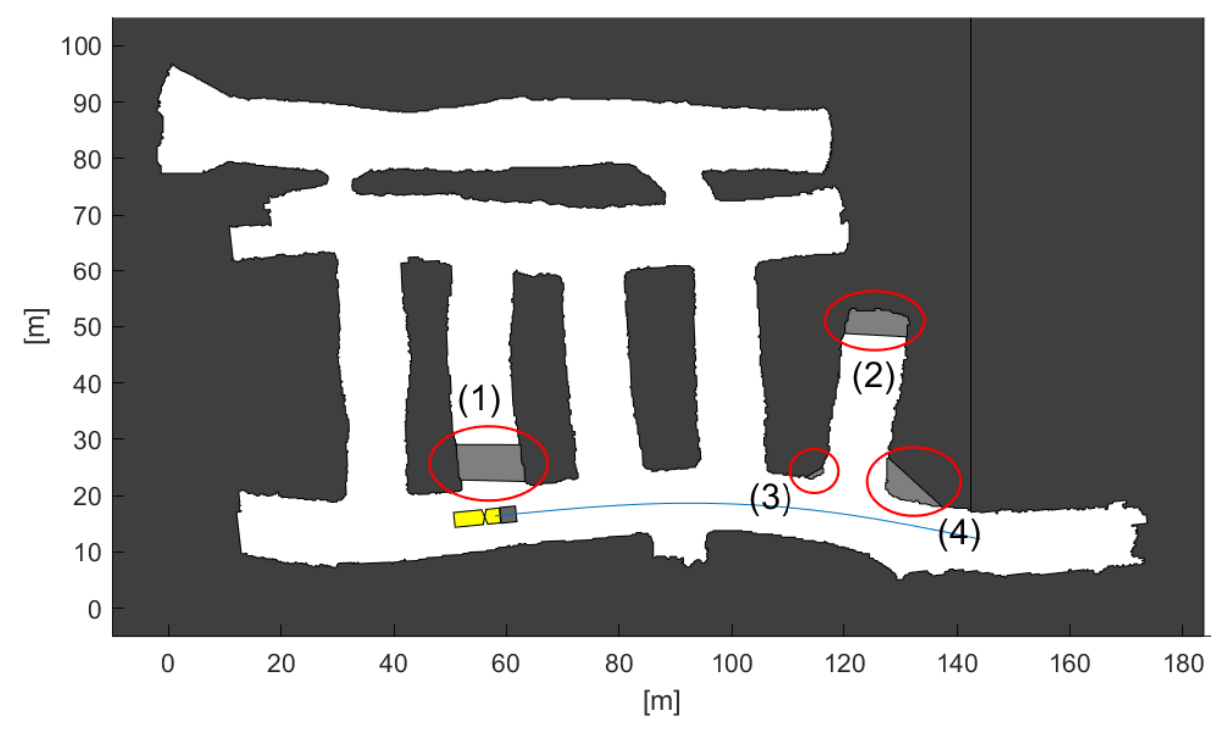

Figure 5.6: The vehicle travels along the blue line starting from left to right. The trajectory both starts and stops with zero velocity and light grey areas show modification done to simulate errors in the map. (1) A tunnel is filled with material since the map was created. (2) The tunnel is shortened as if rocks have fallen down. (3) A small chunk of material is cut-off in a corner. (4) A larger chunk of material is cut-off in a corner.

long tunnels, cross-sections, and irregularities of the walls captured in the LIDAR data. Since the UKF outputs an estimate of the state covariance in each time increment, the radial distance $d_{\max }$ now changes dynamically. For FLIRT where only pure geometric association is used, the threshold is fixed to $0.1 \mathrm{~m}$.

In Figure 5.7(a), the RMSE of the estimated state is presented for each time increment along the trajectory. The poor result for the basic ICP variant [26] could probably be improved by a sophisticated outlier rejection principle. For example, as mentioned in Section 2.4 many variations of the algorithm addressing specific properties do exist, or as discussed in Section 2.5.4, ICP can be used in conjunction with RANSAC to decrease the influence of outliers. However, this adds complexity to the system and is something that is somewhat automatic in the feature extraction approach. Since RANSAC can also be used to boost the performance of the feature extraction approach, a better understanding of the performance of the actual methods is given by comparing the pure versions of them, without adding extra steps.

In Figure 5.7(b)-(f), laser data is still simulated in the original map but the UKF is handed a modified version to perform the position estimate in. This mimics a situation when a tunnel is extended/closed, rocks have fallen down, a corner is worn down, or some other change in the environment that is not updated accordingly in the map. The ICP approach has a relatively high RMSE in this setting. 


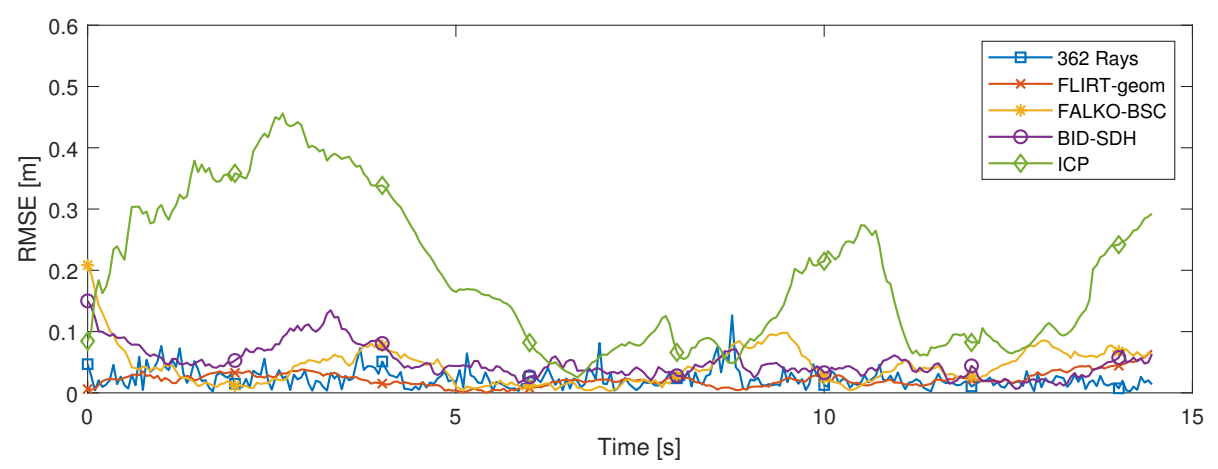

(a) Original map without modifications

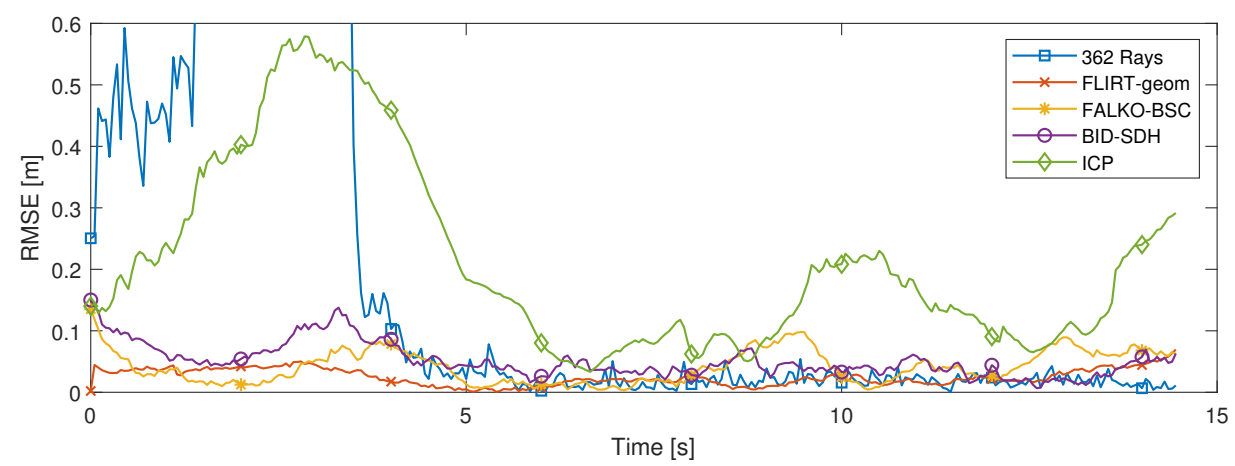

(b) Modification (1) according to Figure 5.6

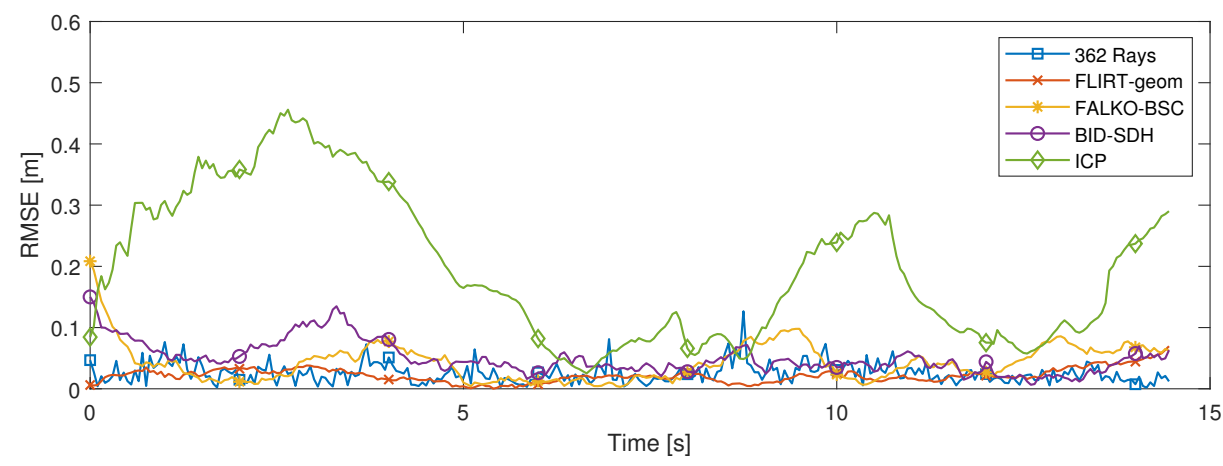

(c) Modification (2) according to Figure 5.6

Figure 5.7: The RMSE of the pose in each time increment along the trajectory in Figure 5.6, estimated with different methods. This is the average result of 10 realizations. 


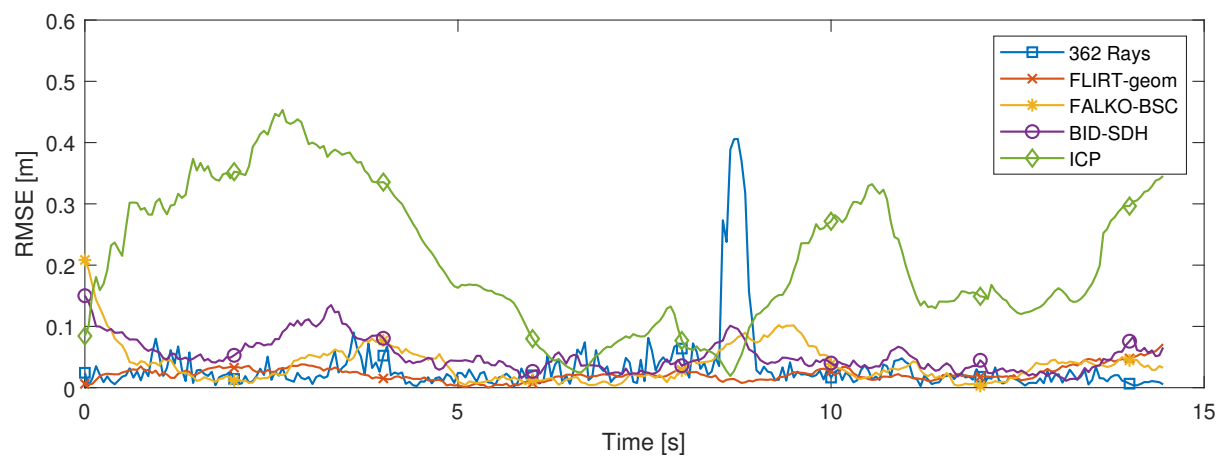

(d) Modification (3) according to Figure 5.6

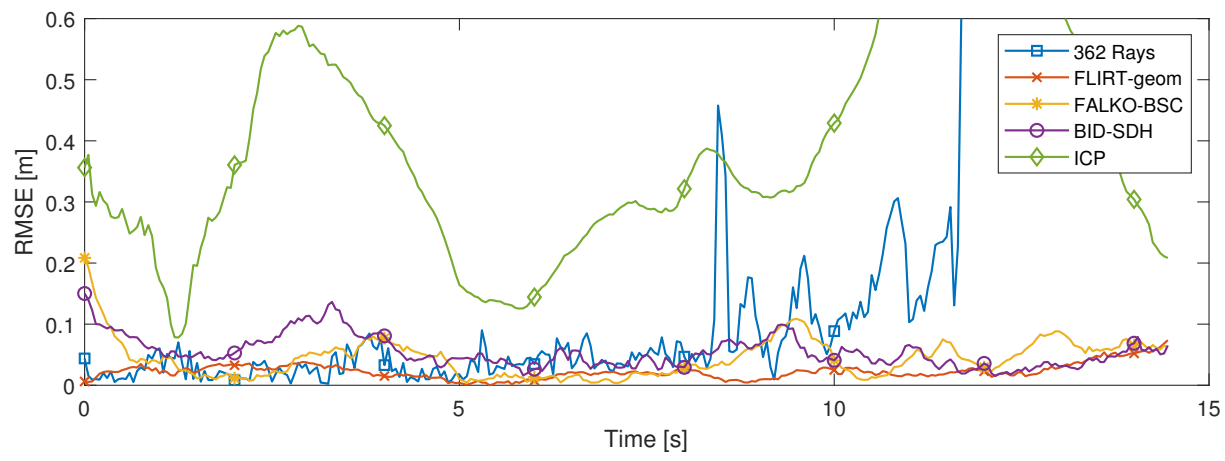

(e) Modification (4) according to Figure 5.6

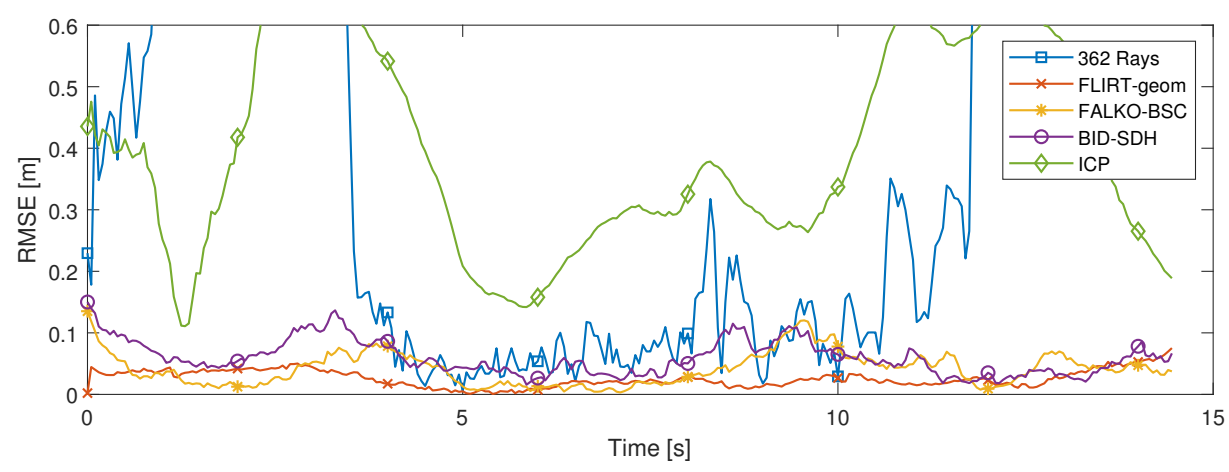

(f) All modifications applied at once. 
Table 5.3: Mean RMSE for all data in Figure 5.7

\begin{tabular}{lc}
\hline Method & Mean RMSE $[\mathrm{m}]$ \\
\hline 362 Rays & 0.68 \\
FLIRT-geom & 0.02 \\
FALKO-BSC & 0.04 \\
BID-SDH & 0.05 \\
ICP & 0.27 \\
\hline
\end{tabular}

It is somewhat robust to small changes, while the performance is considerably worse for larger modifications. The estimates produced by the feature extraction methods are all relatively unaffected by the modifications in the map, while the approach where each ray individually enters the measurement equation is highly sensitive to imperfections in the map. For some of the modifications the filter diverges with this approach.

Out of the feature extraction methods evaluated in this experiment FLIRTgeom performs best, despite the fact that only a pure geometric data association is used. In Table 5.3 the mean RMSE for all maps and time increments are presented for each of the methods, and FLIRT gives an estimate with only half the error of the others.

\subsection{Summary}

In this chapter methods to extract features from 2D LIDAR data have been evaluated for robust and highly accurate position estimation. Three state-of-the-art methods, FLIRT- $\beta$-grid, FALKO-BSC and BID-SDH, have been adapted to fit the scenario of localization in a predefined map. Experiments conducted in the simulated underground mine environment have shown that all of these methods perform better than the point cloud scan matching method ICP, and in parity with the method of matching each ray individually to the map, in situations without map errors. The results also demonstrate that all the feature extraction methods are much more robust to errors and imperfections in the map, simply because no matches are found in those regions. This is an important advantage since the underground mine environment changes dynamically and with a method insensitive to errors, the predefined map is not forced to change accordingly.

In general, the feature extraction methods are sensitive to parameter settings, and all parameters have been manually tuned to fit this particular application. The best performing method is shown to be the FLIRT feature detector in combination with a pure geometric data association with default parameters suggested in [107]. The used nearest neighbor geometric data association could probably be improved by applying a more sophisticated method for outlier rejection, e.g., RANSAC. 


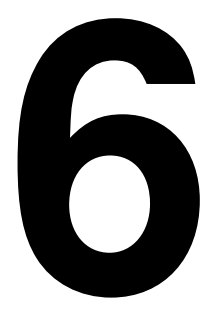

\section{Concluding remarks}

This chapter concludes this thesis by summarizing results and discussing how they contribute to a more robust and computationally efficient localization method. Also possible directions for future work are highlighted.

\subsection{Conclusions}

A positioning system that is highly accurate, reliable, and robust is requested from the mining industry as an enabler for fully autonomous mining systems. Localization methods based on the Bayesian filtering framework have in this thesis been evaluated for this purpose. Key properties, vital for the performance of real-time localization systems in underground mines, have been identified and investigated.

Standard settings of parameter values of a UKF have been shown to perform poorly in the underground mine application. The problem of tuning the design parameters to obtain reliable state estimates has therefore been addressed. By following guidelines and optimization procedures developed in Chapter 3 the performance was substantially improved.

To reduce the dimensionality of the high-dimensional LIDAR data, strategies for selecting subsets of available observations were developed in Chapter 4 . Results showed that using only $20 \%$ of available data is enough to maintain the quality in the state estimates. The information contribution from each individual laser ray in a LIDAR scan have been analyzed, highlighting aspects that are important for a observation to be informative. Knowing the information contribution, strategies for selecting subsets of available measurements, without losing quality in the state estimates, were developed.

Another approach, also reducing the dimensionality of the measurements, is to extract features in the LIDAR data. State-of-the-art feature extraction meth- 
ods for 2D LIDAR data have been evaluated from the perspective of underground mine localization in Chapter 5. This approach has been shown to, in addition to decrease the computational complexity in the filter updates, also provide position estimates resilient to changes in the environment. Errors in the map are automatically ignored with this method. Results from the conducted experiments show that in regions where the map poorly represents the real world, features are not matched and thus omitted for further processing.

The onboard localization system studied in this thesis requires no external infrastructure, which is preferred in the harsh and dynamic environment of the underground mine. The contributions described in this thesis, gives insight to what the main obstacles in obtaining robust position estimates are for this application. Suggestions and solutions are given that specifically improve the utilization of the information contained in 2D LIDAR measurements, resulting in more robust localization methods.

Robust localization in GNSS denied environments is a complex and real problem experienced by practitioners in many fields. The focus of this thesis has been on underground mining; however, the findings and discussions have bearing on many other applications where reliable GNSS is unavailable and LIDAR data is used for positioning in an unstructured environment.

\subsection{Future work}

This thesis has taken steps towards more robust and accurate positioning for underground mines, especially considering the processing of 2D LIDAR data. In the future, 3D LIDAR resulting in even larger amounts of data to process is expected to become standard. The harsh and dirty environment of an underground mine requires rugged hardware, with often limited computational resources compared to standard hardware. To maintain the real-time requirement, sensor selection strategies such as those described in Chapter 4 might be a necessity. A possible direction for future research is thus to extend the strategies developed in Chapter 4 to the $3 \mathrm{D}$ case.

Another interesting topic for future research is the problem with an underground mine being a non-static environment. Tunnels are extended, or closed by a pile of material only to later be re-opened, wall reinforcements can change how a LIDAR perceives a cross-section, and piles in a draw point change structure as material is being moved. The feature extraction approach adopted in Chapter 5 provides a robust solution when the map does not coincide with the real world. Therefore it would be interesting to explore how this approach can be used to enable automatic updates of the map. By considering the features without a match, knowledge can be extracted about what features should have been observed but were not, and what features are new and never observed before. This information can then be used as input to a system that updates the map. 


\section{Bibliography}

[1] M. A. Al-Ammar, S. Alhadhrami, A. Al-Salman, A. Alarifi, H. S. Al-Khalifa, A. Alnafessah, and M. Alsaleh. Comparative survey of indoor positioning technologies, techniques, and algorithms. In Proceedings of the International Conference on Cyberworlds, pages 245-252, Santander, Spain, 2014.

[2] I. Arasaratnam and S. Haykin. Cubature Kalman filters. IEEE Transactions on Automatic Control, 54(6):1254-1269, 2009.

[3] U. Artan, J. A. Marshall, and N. J. Lavigne. Robotic mapping of underground mine passageways. Mining Technology, 120(1):18-24, 2011.

[4] A. E. Assaf, S. Zaidi, S. Affes, and N. Kandil. Accurate sensors localization in underground mines or tunnels. In Proceedings of the IEEE International Conference on Ubiquitous Wireless Broadband (ICUWB), pages 1-6, Montreal, QC, Canada, 2015.

[5] C. Audet and J. E. Dennis. Analysis of generalized pattern searches. SIAM Journal on Optimization, 13(3):889-903, 2002.

[6] T. Bailey, E. M. Nebot, J. K. Rosenblatt, and H. F. Durrant-Whyte. Data association for mobile robot navigation: a graph theoretic approach. In Proceedings of the IEEE International Conference on Robotics and Automation (ICRA), volume 3, pages 2512-2517, San Francisco, CA, USA, 2000.

[7] H. Bay, T. Tuytelaars, and L. Van Gool. SURF: speeded up robust features. In Proceedings of the European Conference on Computer Vision, pages 404-417, Berlin, Heidelberg, 2006. Springer Berlin Heidelberg.

[8] S. Belongie, J. Malik, and J. Puzicha. Shape matching and object recognition using shape contexts. IEEE Transactions on Pattern Analysis and Machine Intelligence, 24(4):509-522, apr 2002.

[9] O. Bengtsson and A. J. Baerveldt. Localization in changing environments estimation of a covariance matrix for the IDC algorithm. In Proceedings of the IEEE/RSJ International Conference on Intelligent Robots and Systems (IROS), volume 4, pages 1931-1937, Maui, HI, USA, 2001. 
[10] P. J. Besl and N. D. McKay. A method for registration of 3-D shapes. IEEE Transactions on Pattern Analysis and Machine Intelligence, 14(2):239-256, 1992.

[11] P. Biber and W. Strasser. The normal distributions transform: a new approach to laser scan matching. In Proceedings of the 2003 IEEE/RSJ International Conference on Intelligent Robots and Systems (IROS), Las Vegas, NV, USA, 2003.

[12] L. Bingrong, Q. Changwen, Z. Zheng, and G. Linpeng. Application of UKF alogrithm in passive localization and tracking. In IEEE 3rd Advanced Information Technology, Electronic and Automation Control Conference (IAEAC), pages 2530-2534, 2018.

[13] C. Bishop. Pattern recognition and machine learning. Springer-Verlag New York, 2006. ISBN 9780387310732.

[14] M. Bosse and R. Zlot. Map matching and data association for large-scale two-dimensional laser scan-based SLAM. The International Journal of Robotics Research, 27(6):667-691, 2008.

[15] M. Bosse and R. Zlot. Keypoint design and evaluation for place recognition in 2D lidar maps. Robotics and Autonomous Systems, 57(12):1211-1224, 2009.

[16] M. Calonder, V. Lepetit, C. Strecha, and P. Fua. BRIEF: binary robust independent elementary features. In Proceedings of the European Conference on Computer Vision, pages 778-792, Berlin, Heidelberg, 2010. Springer Berlin Heidelberg.

[17] M. A. Carreira-Perpiñán and R. S. Zemel. Proximity graphs for clustering and manifold learning. In Proceedings of the $17^{\text {th }}$ International Conference on Neural Information Processing Systems, pages 225-232. MIT Press, 2004.

[18] A. Chehri, P. Fortier, and P. M. Tardif. UWB-based sensor networks for localization in mining environments. Ad Hoc Networks, 7(5):987-1000, 2009.

[19] Y. Chen and G. Medioni. Object modeling by registration of multiple range images. In Proceedings of the IEEE International Conference on Robotics and Automation, volume 3, pages 2724-2729, Sacramento, CA, USA, 1991.

[20] Z. Chen, C. Heckman, S. Julier, and N. Ahmed. Weak in the NEES?: auto-tuning Kalman filters with Bayesian optimization. In Proceedings of the $21^{\text {st }}$ International Conference on Information Fusion (FUSION), pages 1072-1079, Cambridge, UK, 2018.

[21] S. P. Chepuri and G. Leus. Sparsity-promoting sensor selection for nonlinear measurement models. IEEE Transactions on Signal Processing, 63 (3):684-698, 2015. 
[22] H. Chi, K. Zhan, and B. Shi. Automatic guidance of underground mining vehicles using laser sensors. Tunnelling and Underground Space Technology, 27(1):142-148, 2012.

[23] M. Cypriani, G. Delisle, and N. Hakem. Wi-Fi-based positioning in a complex underground environment. Journal of Networks, 10(3), 2015.

[24] M. P. Deisenroth, M. F. Huber, and U. D. Hanebeck. Analytic momentbased gaussian process filtering. In Proceedings of the 26th Annual International Conference on Machine Learning, pages 225-232, Montreal, Quebec, Canada, 2009. Association for Computing Machinery.

[25] M. A. Fischler and R. C. Bolles. Random sample consensus: a paradigm for model fitting with applications to image analysis and automated cartography. Communications of the ACM, 24(6):381--395, 1981.

[26] A. Geiger, P. Lenz, and R. Urtasun. Are we ready for autonomous driving? the KITTI vision benchmark suite. In Proceedings of the IEEE International Conference on Computer Vision and Pattern Recognition, pages 3354-3361, Providence, RI, USA, 2012.

[27] N. Gelfand, L. Ikemoto, S. Rusinkiewicz, and M. Levoy. Geometrically stable sampling for the ICP algorithm. In Proceedings of the $4^{\text {th }}$ International Conference on 3-D Digital Imaging and Modeling (3DIM), Banff, Alta., Canada, 2003.

[28] V. K. Ghorpade, P. Checchin, L. Malaterre, and L. Trassoudaine. Performance evaluation of 3D keypoint detectors for time-of-flight depth data. In Proceedings of the $14^{\text {th }}$ International Conference on Control, Automation, Robotics and Vision, pages 1-6, Phuket, Thailand, 2016. IEEE.

[29] K. Granström. Extended target tracking using PHD filters. PhD thesis, Linköping University, Automatic Control, The Institute of Technology, 2012.

[30] J. Guivant. Victoria Park dataset, 2006. URL http://www-personal. acfr.usyd.edu.au/nebot/victoria_park.htm.

[31] Y. Guo, M. Bennamoun, F. Sohel, M. Lu, and J. Wan. 3D object recognition in cluttered scenes with local surface features: a survey. IEEE Transactions on Pattern Analysis and Machine Intelligence, 36(11):2270-2287, 2014.

[32] F. Gustafsson. Statistical sensor fusion. Studentlitteratur AB, 2018.

[33] F. Gustafsson and G. Hendeby. Some relations between extended and unscented Kalman filters. IEEE Transactions on Signal Processing, 60(2):545555, Feb. 2012.

[34] H.-M. Gutmann. A radial basis function method for global optimization. Journal of Global Optimization, 19:201-227, 2001. 
[35] J. Han, Q. Song, and Y. Q. He. Kalman filter recent advances and applications., chapter Adaptive unscented Kalman filter and its applications in nonlinear control. IntechOpen, 04 2009. ISBN 978-953-307-000-1.

[36] C. G. Harris and M. Stephens. A combined corner and edge detector. In Proceedings of the Alvey Vision Conference, volume 1, pages 147-151, Manchester, UK, 1988.

[37] M. Himstedt, J. Frost, S. Hellbach, H.-J. Bohme, and E. Maehle. Large scale place recognition in 2D LIDAR scans using Geometrical Landmark Relations. In Proceedings of the IEEE/RSJ International Conference on Intelligent Robots and Systems, pages 5030-5035, Chicago, IL, USA, 2014.

[38] K. Ito and K. Xiong. Gaussian filters for nonlinear filtering problems. IEEE Transactions on Automatic Control, 45(5):910-927, 2000.

[39] G. Jekabsons, V. Kairish, and V. Zuravlyov. An analysis of wi-fi based indoor positioning accuracy. Scientific Journal of Riga Technical University. Computer Science, 44(1):131-137, 2011.

[40] C. Jiang, Z. Chen, R. Su, and Y. C. Soh. Group greedy method for sensor placement. IEEE Transactions on Signal Processing, 67(9):2249-2262, 2019.

[41] S. Joshi and S. Boyd. Sensor selection via convex optimization. IEEE Transactions on Signal Processing, 57(2):451-462, 2009.

[42] S. J. Julier. The scaled unscented transformation. In Proceedings of the American Control Conference, volume 6, pages 4555-4559, Anchorage, AK, USA, 2002.

[43] S. J. Julier. The spherical simplex unscented transformation. In Proceedings of the American Control Conference, pages 2430-2434, Denver, CO, USA, 2003.

[44] S. J. Julier and J. K. Uhlmann. A non-divergent estimation algorithm in the presence of unknown correlations. In Proceedings of the American Control Conference, pages 2369-2373, Albuquerque, NM, USA, 1997.

[45] S. J. Julier and J. K. Uhlmann. Reduced sigma point filters for the propagation of means and covariances through nonlinear transformations. In Proceedings of the American Control Conference, volume 2, pages 887892, Anchorage, AK, USA, 2002.

[46] S. J. Julier and J. K. Uhlmann. Unscented filtering and nonlinear estimation. Proceedings of the IEEE, 92(3):401-422, Mar. 2004.

[47] S. J. Julier, J. K. Uhlmann, and H. F. Durrant-Whyte. A new approach for filtering nonlinear systems. In Proceedings of the American Control Conference, pages 1628-1632, 1995. 
[48] S. J. Julier, J. K. Uhlmann, and H. F. Durrant-Whyte. A new method for the nonlinear transformation of means and covariances in filters and estimators. IEEE Transactions on Automatic Control, 45(3), 2000.

[49] F. Kallasi and D. L. Rizzini. Efficient loop closure based on FALKO lidar features for online robot localization and mapping. In Proceedings of the IEEE/RSJ International Conference on Intelligent Robots and Systems (IROS), Daejeon, South Korea, 2016.

[50] F. Kallasi, D. L. Rizzini, and S. Caselli. Fast keypoint features from laser scanner for robot localization and mapping. IEEE Robotics and Automation Letters, 1(1):176-183, 2016.

[51] R. E. Kalman. A new approach to linear filtering and prediction problems. Transactions of the ASME - Journal of Basic Engineering, 82:35-45, 1960.

[52] S. M. Kay. Fundamentals of statistical signal processing - estimation theory. Prentice Hall, 1993.

[53] J. Kennedy and R. Eberhart. Particle swarm optimization. In Proceedings of the ICNN'95 International Conference on Neural Networks, volume 4, pages 1942-1948, Perth, WA, Australia, 1995.

[54] N. Koenig and A. Howard. Design and use paradigms for gazebo, an opensource multi-robot simulator. In Proceedings of the IEEE/RSJ International Conference on Intelligent Robots and Systems (IROS), volume 3, pages 2149-2154, Sendai, Japan, 2004.

[55] T. G. Kolda, R. M. Lewis, and V. Torczon. Optimization by direct search: new perspectives on some classical and modern methods. SIAM Review, 45(3):385-482, 2003.

[56] S. Krig. Computer vision metrics: survey, taxonomy, and analysis, chapter Interest Point Detector and Feature Descriptor Survey, pages 217-282. Apress, Berkeley, CA, 2014.

[57] H.-J. Kwak, D.-H. Lee, J.-M. Hwang, J.-H. Kim, C.-K. Kim, and G.-T. Park. Improvement of the inertial sensor-based localization for mobile robots using multiple estimation windows filter. In Proceedings of the IEEE/RSJ International Conference on Intelligent Robots and Systems, pages 876-881, Vilamoura, Portugal, 2012.

[58] J. Lafferty, A. Mccallum, and F. Pereira. Conditional random fields: probabilistic models for segmenting and labeling sequence data. In Proceedings of the International Conference on Machine Learning (ICML), pages 282289, Williamstown, MA, USA, 2001.

[59] K. Lai, L. Bo, X. Ren, and D. Fox. A large-scale hierarchical multi-view RGB$\mathrm{D}$ object dataset. In Proceedings of the IEEE International Conference on Robotics and Automation, pages 1817-1824, Shanghai China, 2011. 
[60] T. Lefebvre, H. Bruyninckx, and J. De Schutter. Comment on "A new method for the nonlinear transformation of means and covariances in filters and estimators". IEEE Transactions on Automatic Control, 47(8), Aug. 2002.

[61] M. E. Leventon, W. E. L. Grimson, and O. Faugeras. Statistical shape influence in geodesic active contours. In Proceedings of the Conference on Computer Vision and Pattern Recognition (CVPR), volume 1, pages 316323, Hilton Head Island, SC, USA, 2000.

[62] Y. Li and E. B. Olson. Extracting general-purpose features from LIDAR data. In Proceedings of the IEEE International Conference on Robotics and Automation (ICRA), pages 1388-1393, Anchorage, AK, USA, May 2010.

[63] Y. Li and E. B. Olson. Structure tensors for general purpose LIDAR feature extraction. In Proceedings of the IEEE International Conference on Robotics and Automation, Shanghai, China, 2011.

[64] H. Liu, H. Darabi, P. Banerjee, and J. Liu. Survey of wireless indoor positioning techniques and systems. IEEE Transactions on Systems, Man, and Cybernetics-Part C: Applications and Reviews, 37(6):1067-1080, 2007.

[65] L. Ljung. System identification (2nd ed.): Theory for the user. Prentice Hall PTR, USA, 1999. ISBN 0136566952.

[66] D. G. Lowe. Object recognition from local scale-invariant features. In Proceedings of the IEEE International Conference on Computer Vision, volume 2, pages 1150-1157, Kerkyra, Greece, 1999.

[67] D. G. Lowe. Distinctive image features from scale-invariant keypoints. International Journal of Computer Vision, 60:91-110, 2004.

[68] F. Lu and E. Milios. Robot pose estimation in unknown environments by matching 2D range scans. Journal of Intelligent and Robotic Systems, 18 (3):249-275, 1997.

[69] R. Madhavan, M. W. M. G. Dissanayake, and H. F. Durrant-Whyte. Autonomous underground navigation of an LHD using a combined ICPEKF approach. In Proceedings of the IEEE International Conference on Robotics and Automation, volume 4, pages 3703-3708, Leuven, Belgium, 1998.

[70] J. Marshall, A. Bonchis, E. Nebot, and S. Scheding. Robotics in mining, chapter 59, pages 1549-1576. Springer International Publishing, Cham, 2016.

[71] R. Mautz. The challenges of indoor environments and specification on some alternative positioning systems. In Proceedings of the $6^{\text {th }}$ Workshop on Positioning, Navigation and Communication, pages 29-36, Hannover, Germany, 2009. 
[72] K. Meier, S.-J. Chung, and S. Hutchinson. Visual-inertial curve simultaneous localization and mapping: creating a sparse structured world without feature points. Journal of Field Robotics, 35(4):516-544, 2017.

[73] K. Mikolajczyk and C. Schmid. A performance evaluation of local descriptors. IEEE Transactions on Pattern Analysis and Machine Intelligence, 27 (10):1615-1630, 2005.

[74] K. Mikolajczyk, T. Tuytelaars, C. Schmid, A. Zisserman, J. Matas, F. Schaffalitzky, T. Kadir, and L. V. Gool. A comparison of affine region detectors. International Journal of Computer Vision, 65:43-72, 2005.

[75] T. Nemoto, K. Onodera, R. E. Mohan, M. Iwase, and K. Wood. An application of the simultaneous localization and mapping (SLAM) method based on the unscented Kalman filter (UKF) to a reconfigurable quadruped robot with crawling locomotion. In Proceedings of the International Conference on Reconfigurable Mechanisms and Robots (ReMAR), pages 1-8, 2018.

[76] V. Nguyen, A. Martinelli, N. Tomatis, and R. Siegwart. A comparison of line extraction algorithms using 2D laser rangefinder for indoor mobile robotics. In Proceedings of the IEEE/RSJ International Conference on Intelligent Robots and Systems, pages 1929-1934, 2005.

[77] K. Nielsen and G. Hendeby. Sensor management in 2D lidar-based underground positioning. In IEEE $23^{\text {th }}$ Proceedings of the International Conference on Information Fusion, pages 1-6, Virtual conference, July 2020.

[78] C. A. Niestroj, C. Niedringhaus, and K. Nienhaus. Development of an underground positioning system based on the ultra-wideband radio technology. Mining report, 153(1):69-76, 2017.

[79] D. Nitzan, A. E. Brain, and R. O. Duda. The measurement and use of registered reflectance and range data in scene analysis. Proceedings of the IEEE, 65(2):206-220, 1977.

[80] P. Nunez, R. Vazquez-Martin, J. C. del Toro, A. Bandera, and F. Sandoval. Feature extraction from laser scan data based on curvature estimation for mobile robotics. In Proceedings of the IEEE International Conference on Robotics and Automation (ICRA), pages 1167-1172, Orlando, FL, USA, 2006.

[81] J. Paraszczak, A. Gustafson, and H. Schunnesson. Technical and operational aspects of autonomous LHD application in metal mines. International Journal of Mining, Reclamation and Environment, 29(5):391-403, 2015.

[82] L. Pedraza, D. Rodriguez-Losada, F. Matia, G. Dissanayake, and J. V. Miro. Extending the limits of feature-based SLAM with b-splines. IEEE Transactions on Robotics, 25(2):353-366, 2009. 
[83] S. T. Pfister, K. L. Kriechbaum, S. I. Roumeliotis, and J. W. Burdick. Weighted range sensor matching algorithms for mobile robot displacement estimation. In Proceedings of the IEEE International Conference on Robotics and Automation (ICRA), volume 2, pages 1667-1674, Washington, DC, USA, 2002.

[84] S. T. Pfister, S. I. Roumeliotis, and J. W. Burdick. Weighted line fitting algorithms for mobile robot map building and efficient data representation. In Proceedings of the IEEE International Conference on Robotics and Automation, volume 1, pages 1304-1311, Taipei, Taiwan, 2003.

[85] J. C. Ralston, C. O. Hargrave, and D. W. Hainsworth. Localisation of mobile underground mining equipment using wireless ethernet. In $40^{\text {th }}$ IAS Annual Meeting. Conference Record of the Industry Applications Conference, volume 1, pages 225-230, Kowloon, Hong Kong, China, 2005.

[86] F. Ramos, D. Fox, and H. Durrant-Whyte. CRF-Matching: conditional random fields for feature-based scan matching. In Proceedings of the Robotics: science and Systems, Atlanta, GA, USA, 2007.

[87] S. Rao, S. P. Chepuri, and G. Leus. Greedy sensor selection for non-linear models. In Proceedings of the $6^{\text {th }}$ IEEE International Workshop on Computational Advances in Multi-Sensor Adaptive Processing (CAMSAP), pages 241-244, Cancun, Mexico, 2015.

[88] R. G. Regis and C. A. Shoemaker. A stochastic radial basis function method for the global optimization of expensive functions. INFORMS Journal on Computing, 19(4):497-509, 2007.

[89] M. Rhudy, Y. Gu, and M. R. Napolitano. An analytical approach for comparing linearization methods in EKF and UKF. International Journal of Advanced Robotic Systems, 10(4):208, 2013.

[90] J. M. Roberts, E. S. Duff, and P. I. Corke. Reactive navigation and opportunistic localization for autonomous underground mining vehicles. Information Sciences, 145(1):127-146, 2002.

[91] D. Rodriguez-Losada, F. Matia, and R. Galan. Building geometric feature based maps for indoor service robots. Robotics and Autonomous Systems, 54(7):546-558, 2006.

[92] P. L. Rosin. Measuring corner properties. Computer Vision and Image Understanding, 73(2):291-307, 1999.

[93] M. Roth, G. Hendeby, and F. Gustafsson. Nonlinear Kalman filters explained: A tutorial on moment computations and sigma point methods. Journal of Advances in Information Fusion, 11(1):47-70, 2016.

[94] E. Rublee, V. Rabaud, K. Konolige, and G. Bradski. ORB: an efficient alternative to SIFT or SURF. In Proceedings of the International Conference on Computer Vision, pages 2564-2571, Barcelona, Spain, 2011. 
[95] S. Rusinkiewicz and M. Levoy. Efficient variants of the ICP algorithm. In Proceedings of the $3^{\text {rd }}$ International Conference on 3-D Digital Imaging and Modeling, Quebec City, Canada, 2001.

[96] S. Salti, F. Tombari, and L. D. Stefano. A performance evaluation of 3D keypoint detectors. In Proceedings of the International Conference on 3D Imaging, Modeling, Processing, Visualization and Transmission, pages 236-243, Hanghou, China, 2011. doi: 10.1109/3dimpvt.2011.37.

[97] S. Särkkä. Bayesian filtering and smoothing. Institute of Mathematical Statistics Textbooks. Cambridge University Press, 2013.

[98] L. A. Scardua and J. J. da Cruz. Complete offline tuning of the unscented Kalman filter. Automatica, 80:54-61, 2017.

[99] M. Shamaiah, S. Banerjee, and H. Vikalo. Greedy sensor selection: leveraging submodularity. In Proceedings of the $49^{\text {th }}$ IEEE Conference on Decision and Control (CDC), pages 2572-2577, Atlanta, GA, USA, 2010.

[100] J. Shi and C. Tomasi. Good features to track. In Proceedings of the IEEE Conference on Computer Vision and Pattern Recognition, pages 593-600, Seattle, WA, USA, 1994.

[101] P. Smith, I. Reid, and A. J. Davison. Real-time monocular SLAM with straight lines. In Proceedings of the the British Machine Vision Conference, pages 17-26, Edinburgh, UK, 2006. British Machine Vision Association.

[102] O. Straka, J. Duník, and M. Simandl. Unscented Kalman filter with advanced adaptation of scaling parameter. Automatica, 50(10):2657-2664, 2014. ISSN 0005-1098.

[103] A. Tallavajhula. Lidar simulation for robotic application development: modeling and evaluation. PhD thesis, The Robotics Institute Carnegie Mellon University Pittsburgh, May 2018.

[104] S. Thrun, W. Burgard, and D. Fox. Probabilistic robotics. MIT Press, 2005.

[105] L. Thrybom, J. Neander, E. Hansen, and K. Landernas. Future challenges of positioning in underground mines. In Proceedings of the $2^{\text {nd }}$ IFAC Conference on Embedded Systems, Computer Intelligence and Telematics CESCIT, volume 48, pages 222-226, Maribor, Slovania, 2015.

[106] P. Tichavsky, C. H. Muravchik, and A. Nehorai. Posterior Cramer-Rao bounds for discrete-time nonlinear filtering. IEEE Transactions on Signal Processing, 46(5):1386-1396, 1998.

[107] G. D. Tipaldi and K. O. Arras. FLIRT - interest regions for 2D range data. In Proceedings of the IEEE International Conference on Robotics and $\mathrm{Au}$ tomation, pages 3616-3622, Anchorage, AK, USA, 2010. 
[108] R. Turner and C. E. Rasmussen. Model based learning of sigma points in unscented Kalman filtering. Neurocomputing, 80:47-53, 2012. Special Issue on Machine Learning for Signal Processing 2010.

[109] R. Unnikrishnan and M. Hebert. Multi-scale interest regions from unorganized point clouds. In Proceedings of the IEEE Computer Society Conference on Computer Vision and Pattern Recognition Workshops, pages 1-8, Anchorage, AK, USA, 2008.

[110] M. Usman, A. M. Khan, A. Ali, S. Yaqub, K. M. Zuhaib, J. Y. Lee, and C.-S. Han. An extensive approach to features detection and description for 2-D range data using active B-splines. IEEE Robotics and Automation Letters, 4(3):2934-2941, 2019.

[111] R. van der Merwe. Sigma-point Kalman filters for probabilistic inference in dynamic state-space models. PhD thesis, Oregon Health and Science University, 2004.

[112] R. Van der Merwe and E. A. Wan. The square-root unscented Kalman filter for state and parameter-estimation. In Proceedings of the IEEE International Conference on Acoustics, Speech, and Signal Processing, volume 6, pages 3461-3464, Salt Lake City, UT, USA, 2001.

[113] J. Vandorpe, H. V. Brussel, and H. Xu. Exact dynamic map building for a mobile robot using geometrical primitives produced by a $2 \mathrm{D}$ range finder. In Proceedings of the IEEE International Conference on Robotics and $\mathrm{Au}$ tomation (ICRA), volume 1, pages 901-908, Minneapolis, MN, USA, 1996.

[114] M. Veeck and W. Burgard. Learning polyline maps from range scan data acquired with mobile robots. In Proceedings of the IEEE/RSJ International Conference on Intelligent Robots and Systems (IROS), volume 2, pages 1065-1070, Sendai, Japan, 2004.

[115] E. A. Wan and R. V. D. Merwe. The unscented Kalman filter for nonlinear estimation. In Proceedings of the IEEE Adaptive Systems for Signal Processing, Communications, and Control Symposium, Lake Louise, Alberta, Canada, 2000.

[116] J. L. Williams, J. W. Fisher, and A. S. Willsky. Approximate dynamic programming for communication-constrained sensor network management. IEEE Transactions on Signal Processing, 55(8):4300-4311, 2007.

[117] C. Yang, L. Kaplan, and E. Blasch. Performance measures of covariance and information matrices in resource management for target state estimation. IEEE Transactions on Aerospace and Electronic Systems, 43(3):2594-2613, 2012.

[118] A. Yassin, Y. Nasser, M. Awad, A. Al-Dubai, R. Liu, C. Yuen, R. Raulefs, and E. Aboutanios. Recent advances in indoor localization: A survey on theoretical approaches and applications. IEEE Communications Surveys and Tutorials, 19(2):1327-1346, 2017. 
[119] R. Zhan and J. Wan. Iterated unscented Kalman filter for passive target tracking. IEEE Transactions on Aerospace and Electronic Systems, 43(3): 1155-1163, 2007.

[120] H. Zhang, G. Dai, J. Sun, and Y. Zhao. Unscented Kalman filter and its nonlinear application for tracking a moving target. Optik, 124(20):44684471, 2013.

[121] H. Zhang, R. Ayoub, and S. Sundaram. Sensor selection for Kalman filtering of linear dynamical systems: complexity, limitations and greedy algorithms. Automatica, 78:202-210, 2017.

[122] S. Zhang, M. Adams, F. Tang, and L. Xie. Geometrical feature extraction using 2D range scanner. In Proceedings of the $4^{\text {th }}$ International Conference on Control and Automation, pages 901-905, Montreal, Canada, 2003. 


\section{Licentiate Theses \\ Division of Automatic Control \\ Linköping University}

P. Andersson: Adaptive Forgetting through Multiple Models and Adaptive Control of Car Dynamics. Thesis No. 15, 1983.

B. Wahlberg: On Model Simplification in System Identification. Thesis No. 47, 1985.

A. Isaksson: Identification of Time Varying Systems and Applications of System Identification to Signal Processing. Thesis No. 75, 1986.

G. Malmberg: A Study of Adaptive Control Missiles. Thesis No. 76, 1986.

S. Gunnarsson: On the Mean Square Error of Transfer Function Estimates with Applications to Control. Thesis No. 90, 1986.

M. Viberg: On the Adaptive Array Problem. Thesis No. 117, 1987.

K. Ståhl: On the Frequency Domain Analysis of Nonlinear Systems. Thesis No. 137, 1988.

A. Skeppstedt: Construction of Composite Models from Large Data-Sets. Thesis No. 149, 1988.

P. A. J. Nagy: MaMiS: A Programming Environment for Numeric/Symbolic Data Processing. Thesis No. 153, 1988.

K. Forsman: Applications of Constructive Algebra to Control Problems. Thesis No. 231, 1990.

I. Klein: Planning for a Class of Sequential Control Problems. Thesis No. 234, 1990.

F. Gustafsson: Optimal Segmentation of Linear Regression Parameters. Thesis No. 246, 1990.

H. Hjalmarsson: On Estimation of Model Quality in System Identification. Thesis No. 251, 1990.

S. Andersson: Sensor Array Processing; Application to Mobile Communication Systems and Dimension Reduction. Thesis No. 255, 1990.

K. Wang Chen: Observability and Invertibility of Nonlinear Systems: A Differential Algebraic Approach. Thesis No. 282, 1991.

J. Sjöberg: Regularization Issues in Neural Network Models of Dynamical Systems. Thesis No. 366, 1993.

P. Pucar: Segmentation of Laser Range Radar Images Using Hidden Markov Field Models. Thesis No. 403, 1993.

H. Fortell: Volterra and Algebraic Approaches to the Zero Dynamics. Thesis No. 438, 1994.

T. McKelvey: On State-Space Models in System Identification. Thesis No. 447, 1994.

T. Andersson: Concepts and Algorithms for Non-Linear System Identifiability. Thesis No. 448, 1994.

P. Lindskog: Algorithms and Tools for System Identification Using Prior Knowledge. Thesis No. 456, 1994.

J. Plantin: Algebraic Methods for Verification and Control of Discrete Event Dynamic Systems. Thesis No. 501, 1995.

J. Gunnarsson: On Modeling of Discrete Event Dynamic Systems, Using Symbolic Algebraic Methods. Thesis No. 502, 1995.

A. Ericsson: Fast Power Control to Counteract Rayleigh Fading in Cellular Radio Systems. Thesis No. 527, 1995.

M. Jirstrand: Algebraic Methods for Modeling and Design in Control. Thesis No. 540, 1996.

K. Edström: Simulation of Mode Switching Systems Using Switched Bond Graphs. Thesis No. 586, 1996. 
J. Palmqvist: On Integrity Monitoring of Integrated Navigation Systems. Thesis No. 600, 1997.

A. Stenman: Just-in-Time Models with Applications to Dynamical Systems. Thesis No. 601, 1997.

M. Andersson: Experimental Design and Updating of Finite Element Models. Thesis No. 611, 1997.

U. Forssell: Properties and Usage of Closed-Loop Identification Methods. Thesis No. 641, 1997.

M. Larsson: On Modeling and Diagnosis of Discrete Event Dynamic systems. Thesis No. 648, 1997.

N. Bergman: Bayesian Inference in Terrain Navigation. Thesis No. 649, 1997.

V. Einarsson: On Verification of Switched Systems Using Abstractions. Thesis No. 705, 1998.

J. Blom, F. Gunnarsson: Power Control in Cellular Radio Systems. Thesis No. 706, 1998.

P. Spångéus: Hybrid Control using LP and LMI methods - Some Applications. Thesis No. 724, 1998.

M. Norrlöf: On Analysis and Implementation of Iterative Learning Control. Thesis No. 727, 1998.

A. Hagenblad: Aspects of the Identification of Wiener Models. Thesis No. 793, 1999.

F. Tjärnström: Quality Estimation of Approximate Models. Thesis No. 810, 2000.

C. Carlsson: Vehicle Size and Orientation Estimation Using Geometric Fitting. Thesis No. 840, 2000.

J. Löfberg: Linear Model Predictive Control: Stability and Robustness. Thesis No. 866, 2001.

O. Härkegård: Flight Control Design Using Backstepping. Thesis No. 875, 2001.

J. Elbornsson: Equalization of Distortion in A/D Converters. Thesis No. 883, 2001.

J. Roll: Robust Verification and Identification of Piecewise Affine Systems. Thesis No. 899, 2001.

I. Lind: Regressor Selection in System Identification using ANOVA. Thesis No. 921, 2001.

R. Karlsson: Simulation Based Methods for Target Tracking. Thesis No. 930, 2002.

P.-J. Nordlund: Sequential Monte Carlo Filters and Integrated Navigation. Thesis No. 945, 2002.

M. Östring: Identification, Diagnosis, and Control of a Flexible Robot Arm. Thesis No. 948, 2002.

C. Olsson: Active Engine Vibration Isolation using Feedback Control. Thesis No. 968, 2002.

J. Jansson: Tracking and Decision Making for Automotive Collision Avoidance. Thesis No. 965, 2002.

N. Persson: Event Based Sampling with Application to Spectral Estimation. Thesis No. 981, 2002.

D. Lindgren: Subspace Selection Techniques for Classification Problems. Thesis No. 995, 2002.

E. Geijer Lundin: Uplink Load in CDMA Cellular Systems. Thesis No. 1045, 2003.

M. Enqvist: Some Results on Linear Models of Nonlinear Systems. Thesis No. 1046, 2003.

T. Schön: On Computational Methods for Nonlinear Estimation. Thesis No. 1047, 2003.

F. Gunnarsson: On Modeling and Control of Network Queue Dynamics. Thesis No. 1048, 2003.

S. Björklund: A Survey and Comparison of Time-Delay Estimation Methods in Linear Systems. Thesis No. 1061, 2003. 
M. Gerdin: Parameter Estimation in Linear Descriptor Systems. Thesis No. 1085, 2004.

A. Eidehall: An Automotive Lane Guidance System. Thesis No. 1122, 2004.

E. Wernholt: On Multivariable and Nonlinear Identification of Industrial Robots. Thesis No. 1131, 2004.

J. Gillberg: Methods for Frequency Domain Estimation of Continuous-Time Models. Thesis No. 1133, 2004.

G. Hendeby: Fundamental Estimation and Detection Limits in Linear Non-Gaussian Systems. Thesis No. 1199, 2005.

D. Axehill: Applications of Integer Quadratic Programming in Control and Communication. Thesis No. 1218, 2005.

J. Sjöberg: Some Results On Optimal Control for Nonlinear Descriptor Systems. Thesis No. 1227, 2006.

D. Törnqvist: Statistical Fault Detection with Applications to IMU Disturbances. Thesis No. 1258, 2006.

H. Tidefelt: Structural algorithms and perturbations in differential-algebraic equations. Thesis No. 1318, 2007.

S. Moberg: On Modeling and Control of Flexible Manipulators. Thesis No. 1336, 2007.

J. Wallén: On Kinematic Modelling and Iterative Learning Control of Industrial Robots. Thesis No. 1343, 2008.

J. Harju Johansson: A Structure Utilizing Inexact Primal-Dual Interior-Point Method for Analysis of Linear Differential Inclusions. Thesis No. 1367, 2008.

J. D. Hol: Pose Estimation and Calibration Algorithms for Vision and Inertial Sensors. Thesis No. 1370, 2008.

H. Ohlsson: Regression on Manifolds with Implications for System Identification. Thesis No. 1382, 2008.

D. Ankelhed: On low order controller synthesis using rational constraints. Thesis No. 1398, 2009.

P. Skoglar: Planning Methods for Aerial Exploration and Ground Target Tracking. Thesis No. 1420, 2009.

C. Lundquist: Automotive Sensor Fusion for Situation Awareness. Thesis No. 1422, 2009.

C. Lyzell: Initialization Methods for System Identification. Thesis No. 1426, 2009.

R. Falkeborn: Structure exploitation in semidefinite programming for control. Thesis No. 1430, 2010.

D. Petersson: Nonlinear Optimization Approaches to $\mathcal{H}_{2}$-Norm Based LPV Modelling and Control. Thesis No. 1453, 2010.

Z. Sjanic: Navigation and SAR Auto-focusing in a Sensor Fusion Framework. Thesis No. 1464, 2011.

K. Granström: Loop detection and extended target tracking using laser data. Thesis No. 1465, 2011.

J. Callmer: Topics in Localization and Mapping. Thesis No. 1489, 2011.

F. Lindsten: Rao-Blackwellised particle methods for inference and identification. Thesis No. 1480, 2011.

M. Skoglund: Visual Inertial Navigation and Calibration. Thesis No. 1500, 2011.

S. Khoshfetrat Pakazad: Topics in Robustness Analysis. Thesis No. 1512, 2011.

P. Axelsson: On Sensor Fusion Applied to Industrial Manipulators. Thesis No. 1511, 2011.

A. Carvalho Bittencourt: On Modeling and Diagnosis of Friction and Wear in Industrial Robots. Thesis No. 1516, 2012.

P. Rosander: Averaging level control in the presence of frequent inlet flow upsets. Thesis No. 1527, 2012. 
N. Wahlström: Localization using Magnetometers and Light Sensors. Thesis No. 1581, 2013.

R. Larsson: System Identification of Flight Mechanical Characteristics. Thesis No. 1599, 2013.

Y. Jung: Estimation of Inverse Models Applied to Power Amplifier Predistortion. Thesis No. 1605, 2013.

M. Syldatk: On Calibration of Ground Sensor Networks. Thesis No. 1611, 2013.

M. Roth: Kalman Filters for Nonlinear Systems and Heavy-Tailed Noise. Thesis No. 1613, 2013.

D. Simon: Model Predictive Control in Flight Control Design - Stability and Reference Tracking. Thesis No. 1642, 2014.

J. Dahlin: Sequential Monte Carlo for inference in nonlinear state space models. Thesis No. 1652, 2014.

M. Kok: Probabilistic modeling for positioning applications using inertial sensors. Thesis No. 1656, 2014.

J. Linder: Graybox Modelling of Ships Using Indirect Input Measurements. Thesis No. 1681, 2014.

G. Mathai: Direction of Arrival Estimation of Wideband Acoustic Wavefields in a Passive Sensing Environment. Thesis No. 1721, 2015.

I. Nielsen: On Structure Exploiting Numerical Algorithms for Model Predictive Control. Thesis No. 1727, 2015.

C. Veibäck: Tracking of Animals Using Airborne Cameras. Thesis No. 1761, 2016.

N. Evestedt: Sampling Based Motion Planning for Heavy Duty Autonomous Vehicles. Thesis No. 1762, 2016.

H. Nyqvist: On Pose Estimation in Room-Scaled Environments. Thesis No. 1765, 2016.

Y. Zhao: Position Estimation in Uncertain Radio Environments and Trajectory Learning. Thesis No. 1772, 2017.

P. Kasebzadeh: Parameter Estimation for Mobile Positioning Applications. Thesis No. 1786, 2017.

K. Radnosrati: On Timing-Based Localization in Cellular Radio Networks. Thesis No. 1808, 2018.

G. Lindmark: Methods and Algorithms for Control Input Placement in Complex Networks. Thesis No. 1814, 2018.

M. Lindfors: Frequency Tracking for Speed Estimation. Thesis No. 1815, 2018.

D. Ho: Some results on closed-loop identification of quadcopters. Thesis No. 1826, 2018.

O. Ljungqvist: On motion planning and control for truck and trailer systems. Thesis No. 1832, 2019.

P. Boström-Rost: On Informative Path Planning for Tracking and Surveillance. Thesis No. 1838, 2019.

K. Bergman: On Motion Planning Using Numerical Optimal Control. Thesis No. 1843, 2019.

M. Klingspor: Low-rank optimization in system identification. Thesis No. 1855, 2019.

A. Bergström: Timing-Based Localization using Multipath Information. Thesis No. 1867, 2019.

F. Ljungberg: Estimation of Nonlinear Greybox Models for Marine Applications. Thesis No. 1880, 2020.

E. Hedberg: Control, Models and Industrial Manipulators. Thesis No. 1894, 2020.

R. Forsling: Decentralized Estimation Using Conservative Information Extraction. Thesis No. 1897, 2020. 
D. Arnström: On Complexity Certification of Active-Set QP Methods with Applications to Linear MPC. Thesis No. 1901, 2021.

M. Malmström: Uncertainties in Neural Networks: A System Identification Approach. Thesis No. 1902, 2021. 


\section{FACULTY OF SCIENCE AND ENGINEERING}

Linköping studies in science and technology. Licentiate Thesis No.1906, 2021 Department of Electrical Engineering

Linköping University

SE-581 83 Linköping, Sweden

www.liu.se 